\title{
CONLEY'S FUNDAMENTAL THEOREM FOR A CLASS OF HYBRID SYSTEMS
}

\author{
MATTHEW D. KVALHEIM, PAUL GUSTAFSON, AND DANIEL E. KODITSCHEK
}

\begin{abstract}
We establish versions of Conley's (i) fundamental theorem and (ii) decomposition theorem for a broad class of hybrid dynamical systems. The hybrid version of (i) asserts that a globally-defined hybrid complete Lyapunov function exists for every hybrid system in this class. Motivated by mechanics and control settings where physical or engineered events cause abrupt changes in a system's governing dynamics, our results apply to a large class of Lagrangian hybrid systems (with impacts) studied extensively in the robotics literature. Viewed formally, these results generalize those of Conley and Franks for continuoustime and discrete-time dynamical systems, respectively, on metric spaces. However, we furnish specific examples illustrating how our statement of sufficient conditions represents merely an early step in the longer project of establishing what formal assumptions can and cannot endow hybrid systems models with the topologically well characterized partitions of limit behavior that make Conley's theory so valuable in those classical settings.
\end{abstract}

\section{CONTEnTS}

1. Introduction 2

1.1. Contributions and organization of the paper 2

1.2. Related work 3

2. Preliminaries 4

2.1. Two classes of hybrid systems 4

2.2. Hybrid chain equivalence, recurrence, and attracting-repelling pairs 6

3. Main results 10

4. Applications 11

4.1. General classes of MHS to which Theorems 1 and 2 apply 12

5. Examples 14

6. Proofs of the main results 18

6.1. Suspension of a hybrid system 18

6.2. Chain equivalence in the hybrid suspension 25

6.3. Proofs of Theorems 1 and $2 \quad 28$

7. Conclusion 29

Acknowledgments 30

References $\quad 30$

Supplementary Materials (SM) 34

Appendix A. Relationship with selected prior work 34

A.1. Relationship of Definition 1 to [JBK16] 34

A.2. Relationship of Definition 1 to [AS05] 34

A.3. Relationship of Definition 4 to [CGKS19] 34

A.4. Relationship of the relaxed hybrid system and hybrid suspension to prior work 34

Appendix B. Classical suspension of a discrete-time dynamical system 37

Appendix C. Continuous hybrid suspension semiflow implies the trapping guard condition 37

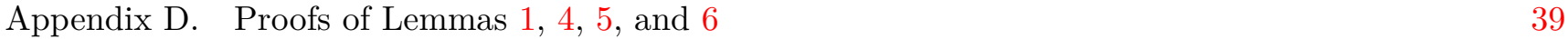

(Kvalheim, Koditschek) School of Engineering and Applied Science, University of Pennsylvania, Philadelphia, PA 19104, USA

(Gustafson) Department of Electrical Engineering, Wright State University, Dayton, OH 45435, USA

E-mail addresses: kvalheim@seas.upenn.edu, paul.gustafson@wright.edu, kod@seas.upenn.edu. 


\section{INTRODUCTION}

In [Nor95], Norton argued that the following two theorems deserve to be called the "Fundamental Theorem of Dynamical Systems."

Theorem ([Con78]). Any continuous flow on a compact metric space decomposes into a chain recurrent part and a gradient-like part. There exists a continuous Lyapunov function which strictly decreases along the flow on the gradient-like part.

Theorem ([Fra88]). The iteration of a homeomorphism of a compact metric space decomposes the space into a chain recurrent part and a gradient-like part. There exists a continuous Lyapunov function which strictly decreases under iteration of the map on the gradient-like part.

See [AN07, Nor95, Rob99] for tutorial accounts.

From the view of applications, these results endow models that achieve them with two important guarantees. First, the decomposition establishes a clear, deterministic notion of steady state behavior that, no matter how complicated its temporal manifestation [Lor64, May76, Hol90], imposes a computationally effective [KMV05] spatial partition into attractor basins [Mil06] whose topology persists under small perturbations. The passage from signal to symbol afforded by such partitions has great value for analyzing natural systems $\left[\mathrm{AKK}^{+}\right.$09, GVdBV03], and has encouraged slowly growing use in the synthesis of engineered systems as well $\left[\mathrm{ACR}^{+} 02, \mathrm{CML}^{+} 07, \mathrm{HCK} 11, \mathrm{HRK12}\right]$. Second, interpreted as a universal converse Lyapunov theorem, global analogue to the classical counterpart addressing a specific basin [Kel15], the long established value for classical [Son89], multistable [FA18] and hybrid control systems theory [GST09] is leveraged by a steadily advancing literature on constructive methods for their eventual feedback closed loops [BK06, GH15]. In our view, one of the most important applications for Lyapunov-expression of basin partitions is their long-proven role in sequential composition [BRK95] and their promise for parallel composition [Cow07, TVDK19], increasing the expressive richness of topologically grounded type theories [AH15] emerging from hybrid dynamical categories that admit them.

1.1. Contributions and organization of the paper. Motivated by problems of robotics and biomechanics, where the making and breaking of contacts intrinsic to most tasks necessitates the introduction of hybrid systems models [Kod21], this paper addresses the question of what hybrid systems models admit a version of Conley's fundamental theorem. We focus on a partial extension of a particularly simple but empirically useful class [JBK16], relative to which a closely related extension can be shown to generate a formal category equipped with the desired compositional operators [CGKS19]. Specifically, we introduce the class of topological hybrid systems (THS) and the subclass of metric hybrid systems (MHS) (Def. 1) that roughly generalizes the model of [JBK16] (see SM §A.1).

After imposing assumptions including the trapping guard condition (Def. 11) we prove an appropriately generalized version of Conley's decomposition theorem (Theorem 1) as well as the existence (Theorem 2) of a globally-defined hybrid complete Lyapunov function (Def. 12). These are our main results. Because we believe the methods used to prove the main results are of independent interest, we provide a rough synopsis of the proof techniques and the underlying new ideas as follows.

Given a suitable MHS $H$ with state space $\mathcal{X}$, we embed $H$ into an MHS $H^{\prime}$ (the relaxed system) with larger state space $\mathcal{X}^{\prime}$ (see Fig. 6). We then topologically "glue" $\mathcal{X}^{\prime}$ to itself along the reset map of $H^{\prime}$ to obtain a metrizable space $\Sigma_{H}$ (the hybrid suspension) equipped with a continuous semiflow $\Phi_{H}$. As will be discussed at greater length in the literature review $(\S 1.2)$, versions of the relaxed hybrid system and hybrid suspension have previously appeared in [JELS99] and [AS05, BGV $\left.{ }^{+} 15\right]$ (see also the discussion at the end of $\S 1.2$ and in SM §A.4.2 and §A.4.3 for more details). Our central new contribution addresses the implications of the version of this construction we have introduced for the nature of steady state behavior in the dynamics it carries. This entails establishing and exploiting several topological and dynamical properties of the hybrid suspension and their relationships to corresponding properties of the original hybrid system $H$ in a manner we now outline.

It is possible to view $\mathcal{X} \subseteq \Sigma_{H}$ as embedded inside $\Sigma_{H}$ in such a way that the image of each execution of $H$ coincides with the intersection with $\mathcal{X}$ of the image of a trajectory of $\Phi_{H}$. Hurley's generalization [Hur95, Hur98] to semiflows of Conley's decomposition and fundamental theorems applies to $\left(\Sigma_{H}, \Phi_{H}\right)$, in 
particular yielding a complete Lyapunov function $V: \Sigma_{H} \rightarrow \mathbb{R}$ for $\Phi_{H}$ whose restriction $\left.V\right|_{\mathcal{X}}$ to $\mathcal{X}$ yields a candidate hybrid complete Lyapunov function for $H$. What remains is to prove that various topologicaldynamical objects associated to $H$ - $\omega$-limit sets, attracting-repelling pairs, and chain equivalence classescoincide with the restrictions to $\mathcal{X} \subseteq \Sigma_{H}$ of corresponding dynamical objects for $\Phi_{H}$. Through various technical arguments we show that this is indeed the case, thereby proving Conley's decomposition and fundamental theorems for $H$ and, in particular, proving that the restriction $\left.V\right|_{\mathcal{X}}$ is indeed a complete Lyapunov function for $H$. For these arguments it turns out to be crucial (for several reasons - see Remarks 15 and 17 and SM $\S \mathrm{A} .4$ for more details) that the hybrid suspension technique differs from the hybrifold technique of [SJSL00, SJLS05].

Our contributions additionally include illustrations of the applicability of our main results by presenting two broad MHS subclasses to which they apply: the smooth exit-boundary guarded MHS (Prop. 2) arising, for example in problems of legged locomotion [BRS15, DBK18]; and an extension (Prop. 3) of the Lagrangian hybrid systems (Cor. 1), a class of models (or near variations thereof) studied in the robotics literature [GAP01, WGK03, AZGS06, PG09, OA10, BCC17, RBCG17]. In contrast, a simple counterexample (Ex. 4, depicted in Fig. 4) demonstrates that the conclusions of our version of Conley's theorems for MHS can fail without the trapping guard condition. Finally, we use two variants of the Hamiltonian bouncing ball model to illustrate how these results apply to mechanical systems which undergo impacts (and to mechanical systems which have only Zeno maximal executions, in the case of the first variant). Bouncing against gravity (Ex. 5) generates an MHS satisfying the trapping guard condition, yielding the Conley decomposition and complete Lyapunov function (Fig. 5) guaranteed by Theorems 1 and 2. In contrast, because linear time invariant vector fields are homogenous, the MHS generated by bouncing losslessly against a Hooke's law spring (Ex. 6) fails the trapping guard condition, so this system does not satisfy the hypotheses of our main theorems; interestingly, however, this example does still satisfy our main theorems' conclusions. We end the paper with some more philosophically motivated remarks concerning the virtue of parsimony arising from these results that generalize both the discrete (Ex. 1) and the continuous (Ex. 2) classical frameworks to unify the common but heretofore distinct assertions of [Con78, Fra88].

The remainder of this paper is organized as follows. After discussing related work below, we introduce the basic definitions and concepts relevant to our main results in $\S 2$. In $\S 3$ we state our main results. In $\S 4$ and $\S 5$, we present the applications and examples (some very specific and some quite general) as just discussed. As discussed above, the proofs of our main results rely on the reduction of suitably guarded MHS to classical dynamical systems on spaces obtained via the hybrid suspension technique described in $\S 6.1$ (see Fig. 6) which generalizes the classical suspension of a discrete-time dynamical system [Sma67, BS02, p. 797, pp. 21-22]. We conclude with brief remarks of a more speculative nature in $\S 7$. Supplementary Materials (SM) §A compares some of our constructions with selected prior work. SM $\S B$ gives a primer on the classical suspension of a discrete-time dynamical system. SM §C makes precise and proves the statement that, for a class of deterministic THS satisfying mild assumptions, the trapping guard condition holds if and only if a continuous hybrid suspension semiflow exists. SM $\S \mathrm{D}$ contains the proofs of various technical lemmas used in the construction of suspension semiflows and well-behaved $(\epsilon, T)$-chains.

1.2. Related work. As reviewed above, for flows on compact metric spaces, Conley proved the existence of a complete Lyapunov function and that the chain recurrent set is the intersection of all attractingrepelling pairs [Con78]. Franks proved the corresponding results for homeomorphisms of compact metric spaces [Fra88]. Hurley extended the decomposition theorem to maps and semiflows on arbitrary metric spaces [Hur95] and proved the existence of complete Lyapunov functions for maps on separable metric spaces [Hur98]. Using Hurley's result, Patrão proved the existence of a complete Lyapunov function for any semiflow on a separable metric space [Pat11]. In the nondeterministic setting, McGehee and Wiandt generalized Franks' results to the setting of iterations of closed relations [MW06, Wia08]; Bronštein and Kopanskii generalized Conley's results to a class of set-valued dynamical systems such as those arising from certain differential inclusions [BK88]. In the stochastic setting, Liu generalized the decomposition and fundamental theorems to random (semi-)dynamical systems such as those arising from stochastic (partial) differential equations [Liu05, Liu07a, Liu07b]. 
Motivated largely by mathematical models occurring in science and engineering, many investigators have worked to generalize results and tools from classical dynamical systems theory to the hybrid setting. Examples include extensions of local [SJLS01] and global [BPS01] structural stability results, contraction analysis [BC18, BLC18], and many theoretical tools concerning periodic orbits [BSKR16] including Floquet theory [BRS15] and the Poincaré-Bendixson theorem [CBC19, CB20, SSJL02].

As described earlier, to prove our results we introduce what we call the hybrid suspension of a THS defined in terms of what we call the relaxed version of a THS. In writing this paper we have become aware that versions of the relaxed system and hybrid suspension have previously appeared in the hybrid systems literature under different names, although (to the best of our knowledge) only for classes of hybrid systems which are formally less general (imposing more structure) than THS and MHS along several dimensions. The relaxed system is essentially an example of a "temporal relaxation" in the sense of [JELS99]. The hybrid suspension $\Sigma_{H}$ of a THS $H$ could be called a "1-relaxed hybrid quotient space" in the terminology of $\left[\mathrm{BGV}^{+} 15\right]$ or a "homotopy colimit" in the terminology of [AS05]. More details are given in SM §A.4.2 and §A.4.3. The hybrid suspension coincides with (a mild generalization of) the "hybrifold" (introduced in [SJSL00, SJLS05]) of the relaxed version of the original hybrid system but, crucially for us, not with the "hybrifold" of the original hybrid system itself; see Remarks 15 and 17 and SM §A.4.1 for more details.

Finally, our definitions of THS and MHS build on a long history of formal approaches to hybrid automata [SJLS05, HTP05, JBK16, Ler16, CGKS19]. Most specifically, our definition of hybrid $(\epsilon, T)$-chains is almost identical to the definition in [CGKS19] for smooth hybrid systems (with one important difference; see SM $\S A .3)$.

\section{Preliminaries}

2.1. Two classes of hybrid systems. Most definitions of hybrid systems in the literature involve variants of smooth manifolds and vector fields. However, in the same way that "the" natural setting for the classical theory of smooth dynamics is given by sufficiently smooth flows generated by vector fields (or by sufficiently smooth maps) on smooth manifolds, "the" natural setting for the classical theory of topological dynamics is given by continuous flows (or semiflows, or continuous maps) on topological (or metric) spaces. Since Conley's theory is part of the theory of topological dynamics, in Def. 1 we define two classes of hybrid systems which we believe provide a natural setting for a topological-dynamical theory of hybrid systems. These definitions have enabled us to obtain in this paper more natural (and general) results than would be obtainable using definitions already existing in the literature. Beyond such aesthetic considerations, we speculate (see §A.4.2-particularly, Footnote 23) that these coarser topological methods may actually be necessary to handle the non-smooth features intrinsic to physically important hybrid dynamics models. Fortunately (see Remark 1), our results apply to many previously defined classes of hybrid systems that represent special cases of our problem setting.

Following [HS06, Sec. 1.3], a local semiflow $\varphi$ on a topological space $\mathcal{F}$ is a map $\varphi$ : $\operatorname{dom}(\varphi) \rightarrow \mathcal{F}$ defined on an open neighborhood $\operatorname{dom}(\varphi) \subseteq[0, \infty) \times \mathcal{F}$ of $\{0\} \times \mathcal{F}$ satisfying the following conditions, with $\varphi^{t}:=\varphi(t, \cdot)$ and $t, s \in[0, \infty):(\mathrm{i}) \varphi^{0}=\mathrm{id}_{\mathcal{F}}$, (ii) $(t+s, x) \in \operatorname{dom}(\varphi) \Longleftrightarrow \operatorname{both}(s, x) \in \operatorname{dom}(\varphi)$ and $\left(t, \varphi^{s}(x)\right) \in \operatorname{dom}(\varphi)$, and (iii) for all $(t+s, x) \in \operatorname{dom}(\varphi), \varphi^{t+s}(x)=\varphi^{t}\left(\varphi^{s}(x)\right)$. Given $x \in \mathcal{F}$, the map $t \mapsto \varphi^{t}(x)$ defined on some interval is called a trajectory of $\varphi$. The local semiflow $\varphi$ is a semiflow if $\operatorname{dom}(\varphi)=[0, \infty) \times \mathcal{F}$.

The following definition follows much of the terminology of [CGKS19], but uses a simpler model for the discrete-time dynamics. ${ }^{1}$ Our definition of topological hybrid systems (THS) uses a more general model for the continuous-time dynamics, i.e., local semiflows on topological spaces instead of vector fields on manifolds. For this reason, our definition of metric hybrid systems (MHS) differs from that of [CGKS19, Def. 2.17]..$^{2}$

\footnotetext{
${ }^{1}$ More specifically, we ignore any underlying graph structure and the fact that there may be various distinct "modes"; see Remark 1 for more details.

${ }^{2}$ Applications-oriented readers might be interested to consult footnote 23 for a brief discussion motivating the (essentially imperative) benefits of adopting this more general framework.
} 


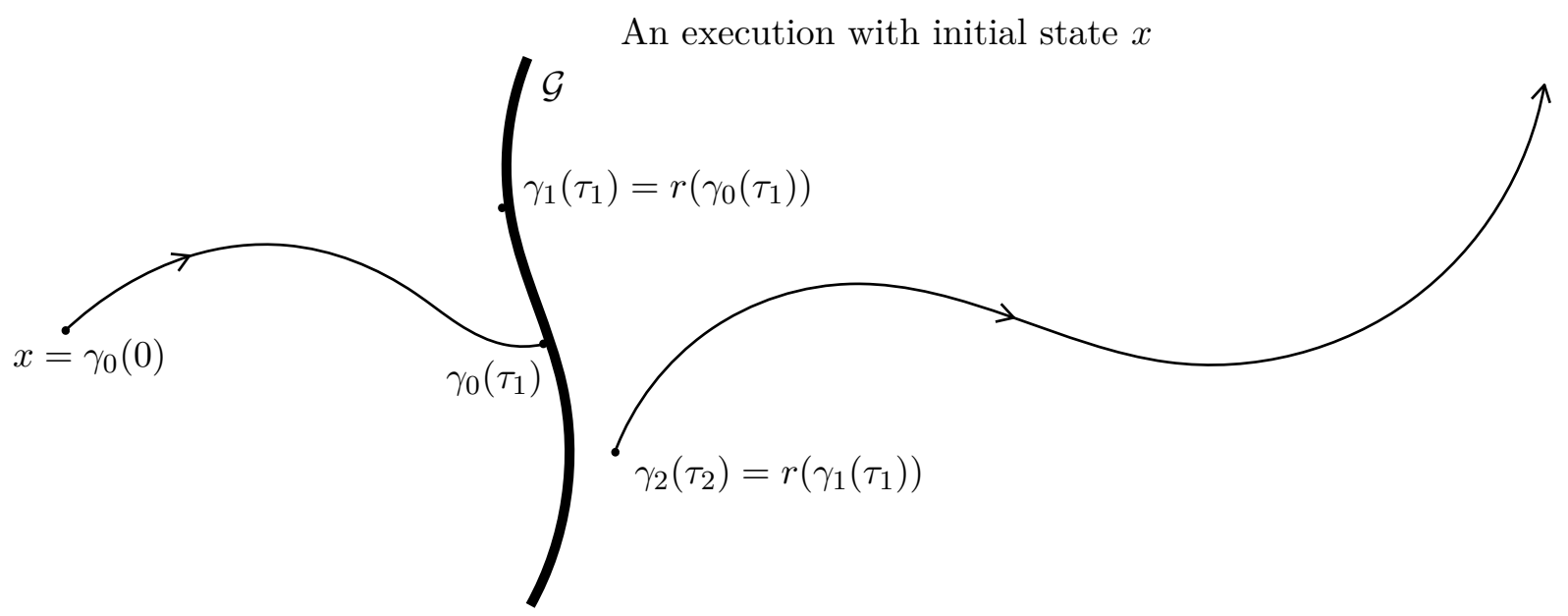

FIgURE 1. An execution in a THS with initial state $x \in \mathcal{F} \subseteq \mathcal{X}$. In this example, $\tau_{1}=\tau_{2}$ and $r\left(\gamma_{0}\left(\tau_{1}\right)\right) \in \mathcal{G}$, highlighting the fact that we allow $r(\mathcal{G}) \cap \mathcal{G} \neq \varnothing$ in Def. 1 .

Definition 1 (Topological and metric hybrid systems). A topological hybrid system (THS) $H=$ $(\mathcal{X}, \mathcal{F}, \mathcal{G}, \varphi, r)$ consists of:

States: a topological state space $\mathcal{X}$ whose points are the possible states of the system.

Continuous-time dynamics: a continuous local semiflow $\varphi$ defined on an open flow set $\mathcal{F} \subseteq \mathcal{X}$. Discrete-time dynamics: a closed guard set $\mathcal{G} \subseteq \mathcal{X}$ equipped with a continuous reset map $r: \mathcal{G} \rightarrow \mathcal{X}$.

If the topology of $\mathcal{X}$ arises from an extended metric dist : $\mathcal{X} \times \mathcal{X} \rightarrow[0,+\infty]$, we say that $(H$, dist) is a metric hybrid system (MHS). (We will usually suppress the extended metric dist from the notation.)

Remark 1. Typical definitions of hybrid systems specify several distinct state spaces (usually smooth manifolds with corners), often called modes, each equipped with its own local semiflow (usually generated by a locally Lipschitz vector field). Each mode may contain a guard set, and discrete transitions from the guard sets to other modes are specified by reset maps (sometimes allowed to be more general relations). Examples of references containing this style of definition include [SJSL00, SJLS01, SSJL02, LJS ${ }^{+}$03, SJLS05, HTP05, BRS15, Ler16, JBK16, BLC18, BC18, CGKS19, CB20, LS20].

At first glance one might incorrectly assume that Def. 1 can encapsulate only hybrid systems consisting of a single mode. However, this is not the case: given a hybrid system as defined in one of the aforementioned references (and having closed guards and continuous reset maps), by defining $\mathcal{X}$ to be the disjoint union of the modes, $\mathcal{F}$ to be the disjoint union of the flow sets, $\mathcal{G}$ to be the disjoint union of the guard sets, $r: \mathcal{G} \rightarrow \mathcal{X}$ to be the disjoint union of the reset maps, and $\varphi$ to be the disjoint union of the local semiflows, one obtains a THS as in Def. 1. If additionally each of the modes of the given hybrid system is equipped with a compatible extended metric (by the Urysohn metrization theorem [Mun00, Thm 34.1], such a metric always exists if each mode is a smooth, paracompact manifold with corners), then one further obtains an MHS as in Def. 1 by leaving the distance between points in the same mode unchanged and defining the distance between points in distinct modes to be infinite. Thus, our results (including Theorems 1 and 2) can be applied to such hybrid systems, as long as they satisfy the relevant additional hypotheses.

Definition 2. Given a THS $H=(\mathcal{X}, \mathcal{F}, \mathcal{G}, \varphi, r)$, an execution in $H$ is a tuple $\chi=(N, \tau, \gamma)$ of

2.1. Jump times: a nondecreasing sequence $\tau=\left(\tau_{j}\right)_{j=0}^{N+1} \subseteq \mathbb{R} \cup\{+\infty\}$ where $N \in \mathbb{N}_{\geq 0} \cup\{+\infty\}$, $\tau_{0}=0$, and $\left(\tau_{j}\right)_{j=0}^{N} \subseteq \mathbb{R}$.

2.2. Flow arcs: a sequence of continuous maps $\gamma=\left(\gamma_{j}: T_{j} \rightarrow \mathcal{X}\right)_{j=0}^{N}$ with $\left[\tau_{j}, \tau_{j+1}\right) \subseteq T_{j} \subseteq\left[\tau_{j}, \tau_{j+1}\right] \cap \mathbb{R}$, $\gamma_{j}\left(\left[\tau_{j}, \tau_{j+1}\right)\right) \subseteq \mathcal{F}$, and such that the restriction $\left.\gamma_{j}\right|_{\left[\tau_{j}, \tau_{j+1}\right)}:\left[\tau_{j}, \tau_{j+1}\right) \rightarrow \mathcal{F}$ is a trajectory segment for the local semiflow $\varphi$. For all $0 \leq j<N$, we additionally require that $T_{j}=\left[\tau_{j}, \tau_{j+1}\right], \gamma_{j}\left(\tau_{j+1}\right) \in \mathcal{G}$, and $\gamma_{j+1}\left(\tau_{j+1}\right)=r\left(\gamma_{j}\left(\tau_{j+1}\right)\right)$. 
We call $\gamma_{0}(0)$ the initial state of $\chi$. If $N<\infty$ and $\tau_{N+1} \in T_{N}$, we call $\gamma_{N}\left(\tau_{N+1}\right)$ the final state of $\chi$. We define the stop time of $\chi$ to be

$$
\tau^{\text {stop }}:=\left\{\begin{array}{ll}
\tau_{N+1} & N<\infty \\
\lim _{j \rightarrow \infty} \tau_{j} & N=\infty
\end{array} .\right.
$$

If the stop time of $\chi$ is infinite, we say that $\chi$ is an infinite execution. ${ }^{3}$ If the stop time of $\chi$ is finite but $\chi$ has infinitely many jumps $(N=\infty)$, we say that $\chi$ is a Zeno execution. We say that $\chi=(N, \tau, \gamma)$ is a maximal execution if, for any execution $\chi^{\prime}=\left(N^{\prime}, \tau^{\prime}, \gamma^{\prime}\right)$ with $\gamma_{0}^{\prime}(0)=\gamma_{0}(0)$ and each $\gamma_{j}$ equal to $\left.\gamma_{j^{\prime}}^{\prime}\right|_{T_{j}}$ for some $j^{\prime} \in\left\{0, \ldots, N^{\prime}\right\}, \chi=\chi^{\prime}$.

We denote the set of executions in $H$ and executions with initial state $x \in \mathcal{X}$ by $\mathcal{E}_{H}$ and $\mathcal{E}_{H}(x)$, respectively. For $\chi=(N, \tau, \gamma) \in \mathcal{E}_{H}$ and any $t \in \bigcup_{j=0}^{N} T_{j}$, we write $\chi(t)=\left\{\gamma_{j}(t) \mid 0 \leq j<N+1, t \in T_{j}\right\}$. We emphasize that $\chi(t)$ is generally a set with multiple elements if $t \in\left\{\tau_{1}, \ldots, \tau_{N}\right\}$, but otherwise $\chi(t)$ is a singleton.

Remark 2. An important point concerning Def. 2 is that, if $\gamma_{j}\left(\tau_{j}\right) \in \mathcal{G}$ for some $0 \leq j \leq N$, then it is possible that $\tau_{j+1}=\tau_{j}$, i.e., an instantaneous reset might occur (and must occur if $H$ is deterministic; see Def. 3 below). In this case, the condition $\gamma_{j}\left(\left[\tau_{j}, \tau_{j+1}\right)\right)=\varnothing \subseteq \mathcal{F}$ is satisfied vacuously. A similar remark applies to Def. 4 below.

We also note that, since the domain of the final arc of an execution is not required to be closed, Def. 2 allows the final arc to be a trajectory of $\varphi$ which "blows up" or "escapes" in finite time (i.e., it cannot be extended to a $\varphi$ trajectory defined for all nonnegative time; see [HS06, Sec. 1.3] for the latter terminology). However, our main results concerning THS $H=(\mathcal{X}, \mathcal{F}, \mathcal{G}, \varphi, r)$ include the assumption that every maximal execution of $H$ is infinite or Zeno, and this assumption implies that $\varphi$ trajectories may only "artificially" escape $\mathcal{F}$ in finite time by converging to a limit in $\mathcal{G}$.

As in [CGKS19, Rem. 2.16], our definition of execution allows for infinitely many jumps in finite time (Zeno executions), but does not allow for subsequent execution after the stop time. In particular, while we do allow Zeno executions, in this paper we do not explicitly consider continuations of Zeno executions past the stop time. (Zeno continuations are considered, e.g., in [JELS99, AZGS06, OA10, JBK16, GS16].)

Remark 3. If $x \in \mathcal{X} \backslash(\mathcal{F} \cup \mathcal{G})$, then Def. 2 implies that the only execution $\chi=(N, \tau, \gamma) \in \mathcal{E}_{H}(x)$ is the trivial execution: $N=0, \tau=(0,0), \gamma_{0}:\{0\} \rightarrow\{x\}$. It follows that, if every $x \in \mathcal{X}$ has an execution $\chi \in \mathcal{E}_{H}(x)$ which is not trivial, then $\mathcal{X}=\mathcal{F} \cup \mathcal{G}$. In particular, if every $x \in \mathcal{X}$ has an infinite or Zeno execution $\chi \in \mathcal{E}_{H}(x)$, it follows that $\mathcal{X}=\mathcal{F} \cup \mathcal{G}$.

Definition 3. A THS $H=(\mathcal{X}, \mathcal{F}, \mathcal{G}, \varphi, r)$ is deterministic if $\mathcal{G} \cap \mathcal{F}=\varnothing$. A THS $H$ is nonblocking if for every $x \in \mathcal{X}$ there is an infinite execution starting at $x$.

For a deterministic THS, maximal executions are unique; cf. [CGKS19, Prop. 2.21]. This justifies the terminology. For a deterministic THS, we use the notation $\chi_{x}$ to denote the unique maximal execution in $\mathcal{E}_{H}(x)$.

Most of our results do not assume the nonblocking condition; rather, most of our results (including Theorems 1 and 2) assume the weaker condition that all maximal executions of a given THS are either infinite or Zeno.

2.2. Hybrid chain equivalence, recurrence, and attracting-repelling pairs. The following definition of $(\epsilon, T)$-chains is similar to [CGKS19, Def. 2.18] (see Remark 4 and SM $\S A .3$ for a comparison). As we show below, the corresponding Conley relation generalizes the classical notions for discrete-time and continuous-time systems on compact metric spaces. We recommend the reader glance at Fig. 2 before

\footnotetext{
${ }^{3}$ Our definition of "infinite execution" follows that of [CGKS19, Def. 2.13]. However, this definition differs from that of $\left[\mathrm{LJS}^{+}\right.$03, p. 4], which refers to both executions having infinite stop time and Zeno executions as "infinite." Since our Def. 3 and [LJS ${ }^{+}$03, Def. III.1] both define "nonblocking" hybrid systems to be those for which all maximal executions are "infinite," our definitions of "nonblocking" thus also differ in meaning. Our definition of "nonblocking" differs from that of [JBK16, Def. 4] in precisely the same way, although the latter reference does not introduce the "infinite execution" terminology.
} 


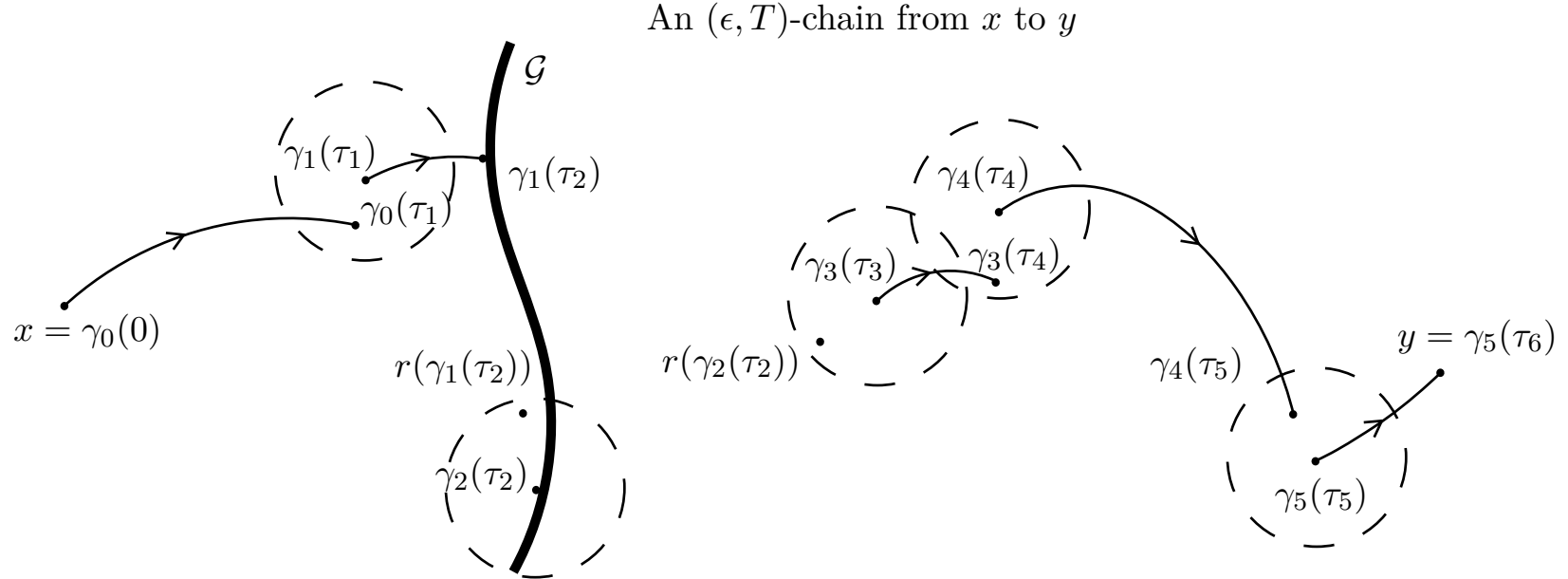

Figure 2. An $(\epsilon, T)$-chain from $x \in \mathcal{X}$ to $y \in \mathcal{X}$ with $N=5$. In this example, both $x, y \in \mathcal{F} \subseteq \mathcal{X}$. All open balls are of radius $\epsilon$, and we have $\tau_{1},\left(\tau_{4}-\tau_{1}\right),\left(\tau_{5}-\tau_{4}\right) \geq T$, but $\left(\tau_{4}-\tau_{3}\right)<T$. In this example, the subsequence $\left(\eta_{k}\right)_{k=0}^{M}$ is given by $\eta_{0}=0, \eta_{1}=1, \eta_{2}=4$, and $\eta_{3}=5$ so that there are $M=3$ continuous-time jumps. Note that the final arc is not required to be degenerate (a single point), in contrast with the classical definitions for continuous-time (semi-)dynamical systems [Con78, Hur95]; however, Ex. 2 shows that this difference is immaterial in the continuous-time setting as far as the Conley relation (Def. 5) is concerned. Note also that the "double jump" $r\left(\gamma_{2}\left(\tau_{2}\right)\right) \rightsquigarrow \gamma_{3}\left(\tau_{3}\right), \gamma_{3}\left(\tau_{4}\right) \rightsquigarrow \gamma_{4}\left(\tau_{4}\right)$ is permitted, even though $\left(\tau_{4}-\tau_{3}\right)<T$, because $\left(\tau_{4}-\tau_{1}\right)=\left(\tau_{\eta_{2}}-\tau_{\eta_{1}}\right) \geq T$. Later in this paper we will also consider nice $(\epsilon, T)$-chains in which "double jumps" are not allowed; see Def. 15 and Fig. 7.

reading the formal definition. We remark that $(\epsilon, T)$-chains can be viewed informally as "executions with errors," with the values of $\epsilon, T$ determining the admissible errors.

Definition 4. Given an MHS $H=(\mathcal{X}, \mathcal{F}, \mathcal{G}, \varphi, r)$ and $\epsilon, T \geq 0$, an $(\epsilon, T)$-chain in $H$ is a tuple $\chi=$ $(N, \tau, \eta, \gamma)$ of

4.1. Jump times: a nondecreasing sequence $\tau=\left(\tau_{j}\right)_{j=0}^{N+1} \subseteq \mathbb{R}$ where $N \in \mathbb{N}_{\geq 1}$ and $\tau_{0}=0$.

4.2. Continuous jump times: a subsequence $\left(\tau_{\eta_{k}}\right)_{k=0}^{M}$ of $\tau$ such that $\eta_{0}=0, \eta_{M} \leq N$, and $\tau_{\eta_{k}}-\tau_{\eta_{k-1}} \geq T$ for all $k \geq 1$.

4.3. Flow arcs: a sequence $\left(\gamma_{j}\right)_{j=0}^{N}$ of continuous maps $\gamma_{j}:\left[\tau_{j}, \tau_{j+1}\right] \rightarrow \mathcal{X}$ such that $\gamma_{j}\left(\left[\tau_{j}, \tau_{j+1}\right)\right) \subseteq \mathcal{F}$ and the restriction $\left.\gamma_{j}\right|_{\left[\tau_{j}, \tau_{j+1}\right)}:\left[\tau_{j}, \tau_{j+1}\right) \rightarrow \mathcal{F}$ is a trajectory segment for the local semiflow $\varphi$. In addition, we require the following:

4.3.1. Continuous-time jump condition: If $j=\eta_{k}$ for some $k \geq 1$, then $\gamma_{j-1}\left(\tau_{j}\right) \in \mathcal{F}$ and

$$
\operatorname{dist}\left(\gamma_{j}\left(\tau_{j}\right), \gamma_{j-1}\left(\tau_{j}\right)\right) \leq \epsilon
$$

4.3.2. Reset jump condition: If $1 \leq j \leq N$ and $j \neq \eta_{k}$ for any $k$, then $\gamma_{j-1}\left(\tau_{j}\right) \in \mathcal{G}$ and

$$
\operatorname{dist}\left(\gamma_{j}\left(\tau_{j}\right), r\left(\gamma_{j-1}\left(\tau_{j}\right)\right)\right) \leq \epsilon
$$

As in Def. 2, we call $\gamma_{0}(0)$ the initial state of $\chi$ and $\gamma_{N}\left(\tau_{N+1}\right)$ the final state of $\chi$. We denote the set of $(\epsilon, T)$-chains in $H,(\epsilon, T)$-chains with initial state $x$, and $(\epsilon, T)$-chains with initial state $x$ and final state $y$ by $\mathcal{C} h_{H}^{\epsilon, T}, \mathcal{C} h_{H}^{\epsilon, T}(x)$ and $\mathcal{C} h_{H}^{\epsilon, T}(x, y)$, respectively. For $\chi=(N, \tau, \eta, \gamma) \in \mathcal{C} h_{H}^{\epsilon, T}$ and $0 \leq t \leq \tau_{N+1}$, we write $\chi(t)=\left\{\gamma_{j}(t) \mid 0 \leq j \leq N, \tau_{j} \leq t \leq \tau_{j+1}\right\}$.

Remark 4. Note that, unlike Def. 2 (and [CGKS19, Def. 2.18]), Def. 4 requires that $N \geq 1$. I.e., we require that an $(\epsilon, T)$-chain consist of at least two arcs. 
Definition 5 (Hybrid Conley relation). Let $H=(\mathcal{X}, \mathcal{F}, \mathcal{G}, \varphi, r)$ be an MHS. The (hybrid) Conley relation $\mathcal{C} h_{H} \subseteq \mathcal{X} \times \mathcal{X}$ is defined by

$$
(x, y) \in \mathcal{C} h_{H} \Longleftrightarrow \text { for all } \epsilon, T>0: \mathcal{C} h_{H}^{\epsilon, T}(x, y) \neq \varnothing .
$$

As is standard with relations, we sometimes use the more intuitive notation $\mathcal{C} h_{H}(x, y)$ in place of $(x, y) \in$ $\mathcal{C} h_{H}$.

Remark 5. Let $H=(\mathcal{X}, \mathcal{F}, \mathcal{G}, \varphi, r)$ be a THS such that $\mathcal{X}$ is metrizable and compact. Then- since all compatible extended metrics on a compact metrizable space are uniformly equivalent - the Conley relation is independent of the choice of compatible metric on $\mathcal{X}$ making $H$ into an MHS. Since the hypotheses for our main results (Theorems 1 and 2) include the assumption that $\mathcal{X}$ is compact, the specific choice of extended metric is immaterial for the majority of our purposes in this paper.

The following two examples show that our hybrid Conley relation generalizes the classical notions in the discrete-time and continuous-time settings.

Example 1. Discrete-time (semi-)dynamical systems given by iterating a continuous map are instances of THS $H=(\mathcal{X}, \mathcal{F}, \mathcal{G}, \varphi, r)$ where $\mathcal{X}=\mathcal{G}$ and $\mathcal{F}=\varnothing$. If $H$ is also an MHS, then for any chain $\chi=$ $(N, \tau, \eta, \gamma) \in \mathcal{C} h_{H}^{\epsilon, T}(x, y)$, we always have $\left(\eta_{k}\right)=(0)$ and $\tau_{j}=0$ for all $j$ (because time never elapses). Each $\operatorname{arc} \gamma_{j}$ is degenerate and corresponds to a single point. Thus $T$ is irrelevant, and we recover the usual notion of an $\epsilon$-chain for a discrete-time system which can also be specified by the more standard notation

$$
\chi=\left(x_{0}=x, x_{1}, \ldots, x_{N}=y\right),
$$

where $x_{i}=\gamma_{i}\left(\tau_{i}\right)=\gamma_{i}(0)$ for all $0 \leq i \leq N$. Thus, our notion of $(\epsilon, T)$-chain restricts to the classical notion when $H$ is a discrete-time system.

Example 2. Suppose $H=(\mathcal{X}, \mathcal{F}, \mathcal{G}, \varphi, r)$ is a continuous-time (semi-)dynamical system given by a continuous semiflow. That is, $\mathcal{X}=\mathcal{F}$ and $\mathcal{G}=\varnothing$. Then if $H$ is also an MHS, the classical notion of an $(\epsilon, T)$-chain for a semiflow [Con78, Hur95] is usually expressed (modulo indexing conventions) as a tuple

$$
\chi=\left(x_{0}=x, x_{1}, \ldots, x_{N}=y ; t_{1}, \ldots, t_{N}\right),
$$

where each $t_{i} \geq T$ and $\operatorname{dist}\left(\varphi^{t_{i}}\left(x_{i-1}\right), x_{i}\right)<\epsilon$ for all $1 \leq i \leq N$. It is easy to see that a classical $(\epsilon, T)$-chain always corresponds to an $(\epsilon, T)$-chain as in Def. 4; however, the converse does not hold. This is because a classical $(\epsilon, T)$-chain always ends with a jump [Con78, Hur95] - or equivalently, using the terminology of Def. 4, ends with a degenerate arc - but an $(\epsilon, T)$-chain in the sense of Def. 4 can end with a nondegenerate arc (see Fig. 2).

However, if $\mathcal{X}=\mathcal{F}$ is compact then the corresponding classical Conley relation is equal to the Conley relation $\mathcal{C} h_{H}$. Indeed, if $\epsilon, T>0$, we can use the uniform continuity of $\varphi$ on $[0, T] \times \mathcal{X}$ to pick $\delta \in(0, \epsilon)$ such that $\operatorname{dist}(p, q)<\delta$ implies $\operatorname{dist}\left(\varphi^{t}(p), \varphi^{t}(q)<\epsilon\right.$ for all $t \in[0, T]$. Let $\chi=(N, \tau, \eta, \gamma) \in \mathcal{C} h_{H}^{\delta, T}(x, y)$; if $\tau_{N+1}-\tau_{N} \geq T$, then we can produce a classical $(\epsilon, T)$-chain by simply adding the degenerate arc at $\gamma_{N}\left(\tau_{N+1}\right)$ to $\chi$. If instead $\tau_{N+1}-\tau_{N}<T$, then we can produce a classical $(\epsilon, T)$-chain from $x$ to $y$ by replacing the final arc with the degenerate arc at its terminal point, and extending the penultimate arc by $\tau_{N+1}-\tau_{N}$ (the penultimate arc necessarily exists since $N \geq 1$ by Def. 4 ).

Definition 6 (Hybrid chain recurrent set). Let $H=(\mathcal{X}, \mathcal{F}, \mathcal{G}, \varphi, r)$ be an MHS. The (hybrid) chain recurrent set $R(H) \subseteq \mathcal{X}$ is defined by

$$
R(H)=\left\{x \in \mathcal{X} \mid \mathcal{C} h_{H}(x, x)\right\}
$$

Definition 7 (Hybrid chain equivalence). Let $H=(\mathcal{X}, \mathcal{F}, \mathcal{G}, \varphi, r)$ be an MHS. Two points $x, y \in \mathcal{X}$ are chain equivalent if $\mathcal{C} h_{H}(x, y)$ and $\mathcal{C} h_{H}(y, x)$. The chain equivalence class of $x \in \mathcal{X}$ is the set $\left\{y \in \mathcal{X} \mid \mathcal{C} h_{H}(x, y)\right.$ and $\left.\mathcal{C} h_{H}(y, x)\right\}$. (Note that every chain equivalence class is a subset of $R(H)$.)

The following definition generalizes the definition of $\omega$-limit set in [LJS ${ }^{+}$03, Def. II.7] (which considered $\omega$-limit sets of singletons only), in addition to generalizing two of the standard definitions for discrete-time and continuous-time dynamical systems [BS02, Con78, p. 29, II.4.1.B]. 


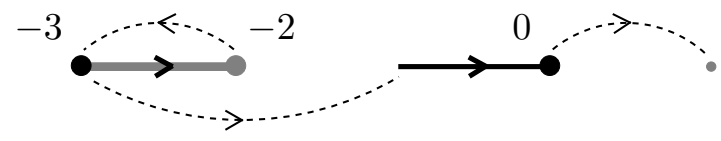

Figure 3. Depicted here is the MHS $H=(\mathcal{X}, \mathcal{F}, \mathcal{G}, \varphi, r)$ discussed in Ex. 3. For this example $\mathcal{X}=[-3,-2] \cup[-1,0] \cup\{1\} \subseteq \mathbb{R}, \mathcal{G}=\{-3,-2,0\}$, and $\mathcal{F}=\mathcal{X} \backslash \mathcal{G}$. The local semiflow $\varphi$ is generated by the vector fields $\frac{\partial}{\partial t}$ on $[-3,-2],-t \frac{\partial}{\partial t}$ on $[-1,0]$, and $0 \frac{\partial}{\partial t}$ on $\{1\}$. $r: \mathcal{G} \rightarrow \mathcal{X}$ is defined by $r(-3)=-1, r(-2)=-3$, and $r(0)=1$. Clearly $H$ is deterministic and nonblocking. For this example, $R(H)=(-3,-2] \cup\{1\}$ (shown in gray). Furthermore, (i,ii) $\omega(-1)=\{0\}$ is neither forward invariant nor contained in $R(H)$, (iii) $R(H)$ is not forward invariant since $r(r(-2))=-1 \notin R(H)$, and (iv) $R(H)$ is not closed in $\mathcal{X}$.

Definition 8 (Hybrid $\omega$-limit set). Let $H=(\mathcal{X}, \mathcal{F}, \mathcal{G}, \varphi, r)$ be a THS. If $U \subseteq \mathcal{X}$, we define the $\omega$-limit set $\omega(U)$ of $U$ via

$$
\omega(U):=\bigcap_{T>0} \operatorname{cl}\left(\bigcup_{x \in U} \bigcup_{\chi=(N, \tau, \gamma) \in \mathcal{E}_{H}(x)}\left\{\gamma_{j}(t) \mid j+t \geq T\right\}\right) .
$$

If $x \in \mathcal{X}$, we define $\omega(x):=\omega(\{x\})$.

Remark 6. If $\mathcal{X}$ is compact, $U \subseteq \mathcal{X}$, and there exists $x \in U$ with an infinite or Zeno execution $\chi \in \mathcal{E}_{H}(x)$, then $\omega(U)$ is a decreasing intersection of nonempty compact sets and is therefore compact and nonempty.

The following result shows that, as in the classical setting, an infinite or Zeno execution with initial state $x$ converges to $\omega(x)$ if $\mathcal{X}$ is compact.

Proposition 1 (Convergence to $\omega$-limit set). Let $H=(\mathcal{X}, \mathcal{F}, \mathcal{G}, \varphi, r)$ be a THS with $\mathcal{X}$ compact. Then for any $x \in \mathcal{X}$ and infinite or Zeno execution $\chi=(N, \tau, \gamma) \in \mathcal{E}_{H}(x)$, we have

$$
\gamma_{j}(t) \rightarrow \omega(x) \quad \text { as } j+t \rightarrow \infty .
$$

That is, for every neighborhood $U \supseteq \omega(x)$, there exists $M>0$ such that $\gamma_{j}(t) \in U$ for all $j+t>M$.

Proof. Suppose not. Then there exists an open neighborhood $U \supseteq \omega(x)$ and subsequences $\left(j_{k}\right)_{k \in \mathbb{N}},\left(t_{k}\right)_{k \in \mathbb{N}}$ with $j_{k}+t_{k} \rightarrow \infty$ such that $\gamma_{j_{k}}\left(t_{k}\right) \in \mathcal{X} \backslash U$ for all $k$. Compactness of $\mathcal{X} \backslash U$ implies that the sequence $\left(\gamma_{j_{k}}\left(t_{k}\right)\right)_{k \in \mathbb{N}}$ has a limit (accumulation) point $y \in \mathcal{X} \backslash U$. But Def. 8 implies that $y \in \omega(x)$, and $\omega(x)$ is disjoint from $\mathcal{X} \backslash U$ by the definition of $U$, so we have a contradiction.

Definition 9 (Forward invariance). Let $H=(\mathcal{X}, \mathcal{F}, \mathcal{G}, \varphi, r)$ be a THS and $S \subseteq \mathcal{X}$ a subset. We say that $S$ is forward invariant if for every $x \in S, \chi \in \mathcal{E}_{H}(x)$, and $t \geq 0, \chi(t) \subseteq S$.

The following example shows that, in spite of Prop. 1, hybrid $\omega$-limit sets and chain recurrent sets can display behavior very different from that of a classical (semi-)dynamical system. In particular, the example shows that the hybrid chain recurrent set need not generally contain the "steady state behavior." Such wild deviance motivates the introduction, in $§ 3$, of the trapping guard condition; a THS satisfying the trapping guard condition does not suffer from such pathologies (see Cor. 2 and 4).

Example 3. Fig. 3 and its caption specify an example which shows that, for a general MHS $H=$ $(\mathcal{X}, \mathcal{F}, \mathcal{G}, \varphi, r)$, the following hold: (i) $\omega$-limit sets need not be forward invariant, (ii) $\omega$-limit sets need not be contained in $R(H)$, (iii) $R(H)$ need not be forward invariant, and (iv) $R(H)$ need not be closed. Furthermore, this example shows that these pathologies can occur even if $\mathcal{X}$ is compact and $H$ is deterministic and nonblocking.

Definition 10 (Hybrid trapping sets and attracting-repelling pairs). Let $H=(\mathcal{X}, \mathcal{F}, \mathcal{G}, \varphi, r)$ be a THS. We say a precompact set $U \subseteq \mathcal{X}$ is a trapping set if the following hold.

- $U$ is forward invariant. 
- There exists $T>0$ such that

$$
\operatorname{cl}\left(\bigcup_{x \in U} \bigcup_{\chi=(N, \tau, \gamma) \in \mathcal{E}_{H}(x)}\left\{\gamma_{j}(t) \mid j+t \geq T\right\}\right) \subseteq \operatorname{int}(U) .
$$

If $U$ is a trapping set, we define the attracting set $A$ determined by $U$ to be $A:=\omega(U)$. We define the repelling set $A^{*}$ dual to $A$ as $A^{*}:=\{x \in \mathcal{X} \mid \omega(x) \cap A=\varnothing\}{ }^{4}$

\section{MAIN RESUltS}

Our main results concern deterministic THS having trapping guards, which we define below using the maximum flow time $\mu: \mathcal{X} \rightarrow[0,+\infty]$ given by

$$
\mu(x):=\left\{\begin{array}{ll}
\sup \{t \in[0, \infty):(t, x) \in \operatorname{dom}(\varphi)\}, & x \in \mathcal{F} \\
0, & x \notin \mathcal{F}
\end{array} .\right.
$$

Definition 11 (Trapping guards). Let $H=(\mathcal{X}, \mathcal{F}, \mathcal{G}, \varphi, r)$ be a deterministic THS. Let $\operatorname{cl}(\operatorname{dom}(\varphi))$ be the closure of $\operatorname{dom}(\varphi)$ in $[0, \infty) \times \mathcal{X}$. We say that $\mathcal{G}$ is a flow-induced retract if there exists a neighborhood $U \subseteq \mathcal{X}$ of $\mathcal{G}$ and a continuous retraction $\rho: U \rightarrow \mathcal{G}\left(\left.\rho\right|_{\mathcal{G}}=\mathrm{id}_{\mathcal{G}}\right)$ such that (i) the $U$-restricted maximum flow time $\left.\mu\right|_{U}: U \rightarrow \mathbb{R}$ is continuous, and (ii) such that $\left.\varphi\right|_{\operatorname{dom}(\varphi) \cap([0, \infty) \times U)}$ admits a unique continuous extension $\widehat{\varphi}$ to $\operatorname{cl}(\operatorname{dom}(\varphi)) \cap([0, \infty) \times U)$ given by

$$
\widehat{\varphi}(t, x)=\left\{\begin{array}{ll}
\varphi^{t}(x), & (t, x) \in \operatorname{dom}(\varphi) \cap([0, \infty) \times U) \\
\rho(x), & x \in U, t=\mu(x)
\end{array} .\right.
$$

We say that $\rho: U \rightarrow \mathcal{G}$ is a flow-induced retraction. If $\mathcal{G}$ is a flow-induced retract, we also say that $H$ has a trapping guard $\mathcal{G}$ or that $H$ satisfies the trapping guard condition.

Remark 7. Note that, in particular, the trapping guard condition rules out the possibility that trajectories of a continuous extension of $\varphi$ "graze" $\mathcal{G}$. However, the trapping guard condition also rules out behavior unrelated to grazing, such as the possibility that $\mathcal{G}$ repel trajectories of $\varphi$ initialized near $\mathcal{G}$.

We justify Def. 11 in several ways. First, Ex. 3 shows that, in the absence of the trapping guard condition, $\omega$-limit and chain recurrent sets need not satisfy many of the properties which are standard in the setting of classical dynamical systems. Second, THS satisfying this condition encompass a wide variety of physically-relevant examples, as shown in the next sections. Third, Ex. 4 demonstrates that our main theorems can fail without the trapping guard condition even for very simple MHS, hence some such condition is necessary. Finally, in Remark 13 and SM $§ \mathrm{C}$ we point out that Def. 11 arises naturally from mathematical considerations.

Our second main result involves the notion of a complete Lyapunov function introduced by Conley [Con78, p. 39]; here we generalize the definition to MHS.

Definition 12 (Hybrid complete Lyapunov function). Let $H=(\mathcal{X}, \mathcal{F}, \mathcal{G}, \varphi, r)$ be an MHS. A (hybrid) complete Lyapunov function for $H$ is a continuous function $L: \mathcal{X} \rightarrow \mathbb{R}$ satisfying the following conditions.

12.1. For every $x \in \mathcal{F} \backslash R(H), \chi \in \mathcal{E}_{H}(x), t>0$, and $y \in \chi(t), L(y)<L(x)$.

12.2. If $x \in \mathcal{G} \backslash R(H)$, then $L(r(x))<L(x)$.

12.3. For all $x, y \in R(H): x$ and $y$ are chain equivalent if and only if $L(x)=L(y)$.

12.4. $L(R(H))$ is nowhere dense in $\mathbb{R}$.

\footnotetext{
${ }^{4}$ For a continuous-time dynamical system given by a flow, Conley showed that $A^{*}$ is also an attracting set for the timereversed flow [Con78, p. 32], so that there is a duality between attracting and repelling sets. For a semiflow, this duality no longer holds as stated because the time-reversed flow is not well-defined. However, we still choose to use the terminology "dual repelling set" for the case of a semiflow (as is somewhat common in the literature [Ryb83, RZ85, FM88]) and, more generally, for the case of a THS. Additionally, we have chosen to use the terminology "attracting and repelling sets" rather than "attractors and repellers" [Con78, II.5.1] because "care is needed since the literature contains many variations on the precise definitions" of the latter [Mil06] (see also [Mil85a, Mil85b]).
} 
In most of the remainder of this paper, we either make or verify Assumptions 1, 2, and 3 below regarding a THS $H=(\mathcal{X}, \mathcal{F}, \mathcal{G}, \varphi, r)$.

Assumption 1. $H$ is deterministic.

Assumption 2. For every $x \in \mathcal{X}$, there is an infinite or Zeno execution starting at $x$.

Assumption 3. $H$ satisfies the trapping guard condition (Def. 11).

We also make the following assumption on $H=(\mathcal{X}, \mathcal{F}, \mathcal{G}, \varphi, r)$ in Theorems 1 and 2.

Assumption 4. $\mathcal{X}$ is compact.

We now state our main results, but postpone the proofs to $§ 6.3$.

Theorem 1 (Conley's decomposition theorem for MHS). Let $H=(\mathcal{X}, \mathcal{F}, \mathcal{G}, \varphi, r)$ be a metric hybrid system satisfying Assumptions 1, 2, 3, and 4. Then the hybrid chain recurrent set $R(H)$ admits a Conley decomposition:

$$
R(H)=\bigcap\left\{A \cup A^{*} \mid A \text { is an attracting set for } H .\right\} .
$$

Furthermore, $x, y \in \mathcal{X}$ are chain equivalent if and only if either $x, y \in A$ or $x, y \in A^{*}$ for every attractingrepelling pair $\left(A, A^{*}\right)$.

Theorem 2 (Conley's fundamental theorem for MHS). Let $H=(\mathcal{X}, \mathcal{F}, \mathcal{G}, \varphi, r)$ be a metric hybrid system satisfying Assumptions 1, 2, 3, and 4. Then there exists a complete Lyapunov function for $H$.

Simple examples illustrating these theorems - including an example which shows that the conclusions can fail without Assumption 3-are given in $\$ 5$. $\$ 4$ contains propositions which guarantee that Theorems 1 and 2 apply to various general classes of hybrid systems appearing in the literature.

Remark 8. As discussed in Ex. 1 and 2, a discrete-time (semi-)dynamical system given by iterating a continuous map is a deterministic THS with $\mathcal{X}=\mathcal{G}$, and a continuous-time (semi-)dynamical system given by a continuous semiflow is a deterministic THS with $\mathcal{X}=\mathcal{F}$. Viewed as hybrid systems, a continuous-time system has only infinite maximal executions, a discrete-time system has only Zeno maximal executions, and in both cases the trapping guard condition is satisfied vacuously. Hence Assumptions 1, 2, and 3 are satisfied in both cases. Additionally, Ex. 2 shows that, although Def. 4 does not specialize to the classical definition of $(\epsilon, T)$-chains in the continuous-time setting [Con78, Hur95], the Conley relations defined using both definitions coincide. Hence Theorems 1 and 2 strictly generalize the corresponding theorems of [Con78, Fra88].

Remark 9. Let $H=(\mathcal{X}, \mathcal{F}, \mathcal{G}, \varphi, r)$ be an MHS not satisfying the hypotheses of Theorems 1 and 2 . If there exists a forward invariant subset $K \subseteq \mathcal{X}$ such that Assumptions 1, 2, 3, and 4 are satisfied by the "restricted" MHS $H_{K}=\left(\mathcal{X} \cap K, \mathcal{F} \cap K, \mathcal{G} \cap K, \varphi_{K},\left.r\right|_{\mathcal{G} \cap K}\right)$ (where $\varphi_{K}$ is the restriction of $\varphi$ to $\operatorname{dom}(\varphi) \cap([0, \infty) \times K))$, then Theorems 1 and 2 can still be be applied to $H_{K}$.

\section{Applications}

This section assumes some basic knowledge of smooth manifold theory [Tu10, Lee13] (and, for Cor. 1, geometric mechanics [MR94]), and can safely be skipped by the reader whose background and/or motivation are lacking. We adopt the definition of "smooth manifold (with boundary)" from [Tu10, p. 48] which allows different connected components to have different dimensions. ${ }^{5}$

\footnotetext{
${ }^{5}$ This variant affords substantial economy of expression. For example, this convention obviates not only the need to introduce the "smooth hybrid manifold" terminology of [BRS15, JBK16, Sec. III.A, Sec. A.4], but also the concomitant technical reasoning required to use it. See also [Tu17] for clarifications.
} 
4.1. General classes of MHS to which Theorems 1 and 2 apply. The following propositions provide general classes of hybrid systems to which Theorems 1 and 2 apply. We refer to hybrid systems satisfying the hypotheses of Prop. 2 as smooth exit-boundary guarded THS, and by smooth exit-boundary guarded MHS if a compatible extended metric on $\mathcal{X}$ is also specified.

We emphasize (as explained in Footnote 5) that we are using the definition of "smooth manifold (with boundary)" from [Tu10, p. 48] which allows different connected components to have different dimensions.

Proposition 2. Let $H=(\mathcal{X}, \mathcal{F}, \mathcal{G}, \varphi, r)$ be a THS for which $\mathcal{X}$ is a smooth manifold with boundary, $\mathcal{G}$ is a clopen subset of $\partial \mathcal{X}, \mathcal{F}:=\mathcal{X} \backslash \mathcal{G}$, and the local semiflow $\varphi$ is generated by a locally Lipschitz ${ }^{6}$ vector field $X$ on $\mathcal{X}$.

Assume that $X$ is non-strictly inward pointing at each point of $\partial \mathcal{X} \backslash \mathcal{G}$, and is non-strictly outward pointing at every point of $\mathcal{G}$. Further assume that, for each $z \in \mathcal{G}$, the maximal integral curve $t \mapsto \sigma(t)$ of $X$ satisfying $\sigma(0)=z$ is not defined for any positive values of $t$.

Then $H$ satisfies Assumptions 1 and 3. If $\mathcal{X}$ is compact, then $H$ also satisfies Assumptions 2 and (trivially) 4. If additionally $\mathcal{X}$ is equipped with any compatible extended metric making $H$ an MHS, then $H$ admits a complete Lyapunov function and $R(H)$ admits a Conley decomposition.

Remark 10. The conditions that (i) $X$ point non-strictly outward on $\mathcal{G}$ and (ii) "the maximal integral curve $t \mapsto \sigma(t)$ of $X$ satisfying $\sigma(0)=z$ is not defined for any positive values of $t$ " (for all $z \in \mathcal{G}$ ) are implied by the stronger assumption, appearing in the hybrid systems literature [BRS15, DBK18, CBC19, CB20], that $X$ be strictly outward pointing at every point of $\mathcal{G}$.

Proof. By definition [Tu10, p. 48] we have that $\mathcal{X}=\bigsqcup_{j \in J} M_{j}$ is a disjoint union of smooth manifolds with each $M_{j}$ of constant dimension. For each $j \in J$ we view $M_{j} \subseteq \mathbb{R}^{n_{j}}$ as a properly embedded submanifold (by Whitney's theorem), extend $X_{j}:=\left.X\right|_{M_{j}}$ arbitrarily to a locally Lipschitz vector field $\widehat{X}_{j}$ on $\mathbb{R}^{n_{j}}$ with local flow $\widehat{\Phi}_{j}: \operatorname{dom}\left(\widehat{\Phi}_{j}\right) \subseteq \mathbb{R} \times \mathbb{R}^{n_{j}} \rightarrow \mathbb{R}^{n_{j}}$, and let $\pi_{2}^{j}: \mathbb{R} \times \mathbb{R}^{n_{j}} \rightarrow \mathbb{R}^{n_{j}}$ be the projection onto the second factor. Then

$$
U_{j}:=M_{j} \cap \pi_{2}^{j}\left(\left(\widehat{\Phi}_{j}\right)^{-1}\left(\mathbb{R}^{n_{j}} \backslash M_{j}\right)\right) \subseteq M_{j}
$$

is an open neighborhood ${ }^{7}$ of $\mathcal{G} \cap M_{j}$ in $M_{j}$ such that (i) for all $x \in U_{j}$ and $T \geq 0$,

$$
\left[\widehat{\Phi}_{j}^{[0, T]}(x) \subseteq \operatorname{cl}\left(U_{j}\right) \subseteq M_{j}\right] \Longrightarrow\left[\widehat{\Phi}_{j}^{[0, T]}(x) \subseteq U_{j}\right],
$$

and (ii) for every $x \in U_{j}$, there exists $t>0$ such that $\widehat{\Phi}_{j}^{t}(x) \in \mathcal{G} \cap M_{j}$. Since also $\mathcal{G} \cap M_{j}$ is closed in $U_{j}$ and every point of $\mathcal{G} \cap M_{j}$ immediately flows into $\mathbb{R}^{n_{j}} \backslash M_{j}$, (i) and (ii) imply (using the terminology of [Con78, p. 24]) that $U_{j}$ is a Wazewski set for $\widehat{\Phi}_{j}$ with eventual exit set $U_{j}$ and immediate exit set $\mathcal{G} \cap M_{j}$. In this case, the proof of Wazewski's theorem [Con78, p. 25] and the definition of the disjoint union topology show that $\mathcal{G}$ is a trapping guard with $\bigsqcup_{j \in J} U_{j}$ the domain of a flow-induced retraction, so Assumption 3 is verified.

Assumption 1 is verified by definition since $\mathcal{F} \cap \mathcal{G}=\varnothing$. If $\mathcal{X}$ is also compact, then (since $X$ points nonstrictly inward at $\partial \mathcal{X} \backslash \mathcal{G}) X$ integral curves either intersect $\mathcal{G}$ or exist for all forward time, so Assumption 2 is verified. Theorems 1 and 2 imply the final statement of the proposition.

Prop. 2 gives one broad class of MHS which satisfy the hypotheses of Theorems 1 and 2. In the following Prop. 3, we present another broad class of MHS to which these theorems also apply. We then specialize Prop. 3 to a class of Lagrangian hybrid systems in Cor. 1. Instances of the latter class of systems (or slight variations thereof) have been studied, for example, in [GAP01, WGK03, AZGS06, PG09, OA10, BCC17, RBCG17]. Looking ahead to §5, this class of systems substantially generalizes the "gravitationalforce bouncing ball" of Ex. 5 (but does not encompass systems like the "spring-force bouncing ball" of Ex. $6)$.

\footnotetext{
${ }^{6}$ This is a well-defined, metric-independent notion which depends only on the smooth structure of $\mathcal{X}$ [KR19, Rem. 1].

${ }^{7}$ This follows since $\mathbb{R}^{n_{j}} \backslash M_{j}$ is open, $\widehat{\Phi}_{j}$ is continuous, $\operatorname{dom}\left(\widehat{\varphi}_{j}\right)$ is open, $\pi_{2}^{j}$ is an open map, and every $z \in \mathcal{G} \cap M_{j}$ immediately flows into $\mathbb{R}^{n_{j}} \backslash M_{j}$; this last property follows from the assumption that the $X$-integral curve through every $z \in \mathcal{G}$ is not defined for any positive times.
} 
The statement of Prop. 3 involves Lie derivatives. Given a smooth manifold $M$, a $C^{k}$ function $\psi: M \rightarrow \mathbb{R}$, and a complete $C^{k}$ vector field $X$ on $M$ with flow $\Phi: \mathbb{R} \times M \rightarrow M$, we let $\mathcal{L}_{X} \psi:=\left.\frac{\partial}{\partial t}\left(\psi \circ \Phi^{t}\right)\right|_{t=0}=d \psi \cdot X$ denote the Lie derivative of $\psi$ along $X$; for all $m \in\{2, \ldots, k\}$ we inductively define $\mathcal{L}_{X}^{m} \psi:=\mathcal{L}_{X}\left(\mathcal{L}_{X}^{m-1} \psi\right)$.

Again using the definition of "smooth manifold (with boundary)" from [Tu10, p. 48] (again, see Footnote 5) we state the following result.

Proposition 3. Let $M$ be a smooth manifold, $k \in \mathbb{N} \cup\{+\infty\}, X$ be a complete $C^{k}$ vector field on $M$ with flow $\Phi: \mathbb{R} \times M \rightarrow M$, and $\psi: M \rightarrow \mathbb{R}$ be a $C^{k}$ function. We define $\mathcal{X}:=\psi^{-1}([0, \infty)), \mathcal{G}:=$ $\psi^{-1}(0) \cap\left(\mathcal{L}_{X} \psi\right)^{-1}((-\infty, 0])$, and $\mathcal{F}:=\mathcal{X} \backslash \mathcal{G}$. We assume given a continuous map $r: \mathcal{G} \rightarrow \mathcal{X}$. Defining the local semiflow $\varphi$ to be the restriction of the flow $\Phi$ to $(\Phi)^{-1}(\mathcal{F}) \cap([0, \infty) \times \mathcal{F})$ and equipping $M$ with any compatible extended metric yields an $\operatorname{MHS} H=(\mathcal{X}, \mathcal{F}, \mathcal{G}, \varphi, r)$. Further assume the following:

- There exists a compact set $K \subseteq \mathcal{X}$ such that $r(\mathcal{G} \cap K) \subseteq K$ and, for all $x \in K$ and $T \geq 0,{ }^{8}$

$$
\left[\Phi^{[0, T]}(x) \subseteq \mathcal{X}\right] \Longrightarrow\left[\Phi^{[0, T]}(x) \subseteq K\right]
$$

- For all $x \in \mathcal{G} \cap K \cap\left(\mathcal{L}_{X} \psi\right)^{-1}(0)$, there exists an integer $m \in[2, k]$ such that

$$
\mathcal{L}_{X}^{1} \psi(x)=\cdots=\mathcal{L}_{X}^{m-1} \psi(x)=0 \quad \text { and } \quad \mathcal{L}_{X}^{m} \psi(x)<0 .
$$

Then the restricted system

$$
H_{K}=\left(\mathcal{X}_{K}, \mathcal{F}_{K}, \mathcal{G}_{K}, \varphi_{K}, r_{K}\right)=\left(\mathcal{X} \cap K, \mathcal{F} \cap K, \mathcal{G} \cap K, \varphi_{K},\left.r\right|_{\mathcal{G} \cap K}\right)
$$

(where $\varphi_{K}$ is the restriction of $\varphi$ to $\operatorname{dom}(\varphi) \cap([0, \infty) \times K)$ ) is well-defined and satisfies Assumptions 1, 2, 3 , and 4 . Hence $H_{K}$ admits a complete Lyapunov function and $R\left(H_{K}\right)$ admits a Conley decomposition.

Proof. The first bulleted condition above implies that $H_{K}$ is a well-defined MHS. $H$ satisfies Assumption 1 since $\mathcal{F} \cap \mathcal{G}=\varnothing$, and $H$ satisfies Assumption 2 since $\mathcal{X}=\mathcal{F} \cup \mathcal{G}$ and $X$ is complete. Hence $H_{K}$ also satisfies Assumptions 1 and 2, and $H_{K}$ trivially satisfies Assumption 4 since $K$ is compact.

We now verify Assumption 3 for $H_{K}$. To do this, we prove that $\mathcal{G} \cap K$ is a trapping guard for $H_{K}$ by constructing a neighborhood $U$ of $\mathcal{G} \cap K$ in $K$ satisfying the conditions of Def. 11. We first define the impact time $\mu: \mathcal{X} \rightarrow[0,+\infty]$ via

$$
\mu(x):=\sup \left\{t \geq 0 \mid \Phi^{[0, t]}(x) \subseteq \mathcal{X}\right\}=\inf \left\{t \geq 0 \mid \Phi^{t}(x) \in M \backslash \mathcal{X}\right\},
$$

where the second equality holds because the mean value theorem, the definition of $\mathcal{G}$, and continuity of $\Phi$ imply that (i) $\Phi^{[0, \epsilon]}(z) \not \mathcal{X}$ for every $z \in \mathcal{G}$ and $\epsilon>0$ and (ii) $\Phi^{\mu(x)}(x) \in \mathcal{G}$ for every $x \in \mathcal{X}$.

We now show that $\mathcal{G} \cap K$ has a neighborhood $U$ in $K$ such that $\mu(x)<\infty$ for every $x \in U$. Suppose not. Then, using (5) and the definition of $\mathcal{X}$, for some $z \in \mathcal{G} \cap K$ there exists a sequence $\left(x_{n}\right)_{n \in \mathbb{N}} \subseteq K$ with $x_{n} \rightarrow z$ and $\psi\left(\Phi^{t}\left(x_{n}\right)\right) \geq 0$ for all $t \geq 0$. This and the fact that $\mathcal{G} \subseteq \psi^{-1}(0)$ imply that $\psi\left(\Phi^{t}(z)\right)=0$ for all $t \geq 0$. Thus, all Lie derivatives of $\psi$ at $z$ vanish, contradicting (4).

Next, we show that $\left.\mu\right|_{U}$ is continuous. For any $x \in U$ and $\epsilon>0$, there exists $T$ in $(\mu(x), \mu(x)+\epsilon)$ with $\Phi^{T}(x) \in M \backslash \mathcal{X}$ by (5). Since $M \backslash \mathcal{X}$ is open in $M$, the continuity of $\Phi^{T}$ yields a neighborhood $V \ni x$ with $\Phi^{T}(V) \subseteq M \backslash \mathcal{X}$. Hence $\mu(V) \subseteq[0, T] \subseteq[0, \mu(x)+\epsilon]$, so $\left.\mu\right|_{U}$ is upper semicontinuous. To show that $\left.\mu\right|_{U}$ is lower semicontinuous, since $\left(\left.\mu\right|_{U}\right)^{-1}(0)=\mathcal{G} \cap K$ it suffices to show that, for every $x \in U \backslash \mathcal{G}$ and every $\epsilon \in(0, \mu(x)), x$ has a neighborhood $V$ with $\mu(V) \subseteq[\mu(x)-\epsilon, \infty)$. Fix $x \in U \backslash \mathcal{G}$. By taking $\epsilon$ smaller if necessary, we may assume that $0<\epsilon<\mu(x)$; pick $T \in(\mu(x)-\epsilon, \mu(x))$. By $(5)$, we have $\Phi^{[0, T]}(x) \subseteq \mathcal{X} \backslash \mathcal{G}$. By the continuity of $\Phi$, compactness of $[0, T]$, and openness of $\mathcal{X} \backslash \mathcal{G}$ in $\mathcal{X}$, there exists a neighborhood $V \ni x$ with $\Phi^{[0, T]}(V) \subseteq \mathcal{X} \backslash \mathcal{G}$, so $\mu(V) \subseteq[T, \infty) \subseteq[\mu(x)-\epsilon, \infty)$ as desired.

It is easy to see that the definition (5) of $\mu$ coincides with that of (1). Since $\left.\mu\right|_{U}$ is continuous and $\left.\mu\right|_{\mathcal{G} \cap K} \equiv 0$, the flow induced-retraction $\rho: U \rightarrow \mathcal{G} \cap K$ defined by $\rho(x):=\Phi^{\mu(x)}(x)$ is continuous. Hence $\widehat{\varphi}_{K}:=\left.\Phi\right|_{\operatorname{cl}\left(\operatorname{dom}\left(\varphi_{K}\right)\right) \cap([0, \infty) \times U)}$ is a continuous extension of $\varphi_{K}$ to $\operatorname{cl}\left(\operatorname{dom}\left(\varphi_{K}\right)\right) \cap([0, \infty) \times U)$ as required in Def. 11, and this extension is unique since $M$ is Hausdorff. Thus, $\mathcal{G} \cap K$ is a trapping guard for $H_{K}$, so $H_{K}$ satisfies Assumptions 1, 2, 3, and 4. Theorems 1 and 2 imply the final statement of the proposition.

\footnotetext{
${ }^{8}$ Conley called this condition positive invariance of $K$ relative to $\mathcal{X}$ [Con78, p. 46].
} 
We now specialize Prop. 3 to show that Theorems 1 and 2 can also be applied to a broad class of mechanical systems with unilateral constraints which undergo impacts, and which generalize the bouncing ball system that we will study in Ex. 5. Slightly generalizing ${ }^{9}$ [AZGS06, Def. 1], we define a hybrid Lagrangian to be a tuple

where:

$$
\mathbf{L}=(Q, L, h),
$$

- $Q$ is a smooth manifold (the configuration space, still assuming the terminological convention discussed in Footnote 5) with tangent bundle $\pi: \mathrm{T} Q \rightarrow Q$,

- $L: \mathrm{T} Q \rightarrow \mathbb{R}$ is a smooth, hyperregular Lagrangian [MR94, Sec. 7.3-7.4] so that the associated Lagrangian vector field $X_{L}: \mathrm{T} Q \rightarrow \mathrm{T}(\mathrm{T} Q)$ is well-defined and smooth (and second order: $\mathrm{T} \pi \circ X_{L}=$ $\left.\operatorname{id}_{\mathrm{T} Q}\right)$, and

- $h: Q \rightarrow \mathbb{R}$ is a smooth function.

We assume that the vector field $X_{L}$ is complete, so that it generates a smooth flow $\Phi: \mathbb{R} \times \mathrm{T} Q \rightarrow \mathrm{T} Q$.

Corollary 1. Let $E: \mathrm{T} Q \rightarrow \mathbb{R}$ be the (total) energy associated to a hybrid Lagrangian $\mathbf{L}=(Q, L, h)$; i.e., $E$ is the pullback of the Hamiltonian associated to $L$ via the Legendre transform [MR94, p. 183, p. 186]. Define $M:=\mathrm{T} Q, X:=X_{L}$, and $\psi:=h \circ \pi ; \mathcal{X}, \mathcal{F}, \mathcal{G}$, and $\varphi$ are then specified as in Prop. 3. Assuming $r: \mathcal{G} \rightarrow \mathcal{X}$ is a given continuous map ${ }^{10}$ and endowing $\mathrm{T} Q$ with any compatible extended metric yields an MHS $H=(\mathcal{X}, \mathcal{F}, \mathcal{G}, \varphi, r)$ associated to $\mathbf{L}$. Further assume the following:

- There exists $E_{0} \in \mathbb{R}$ such that some connected component $K:=S_{E_{0}}$ of $\mathcal{X} \cap E^{-1}\left(\left(-\infty, E_{0}\right]\right)$ is compact and satisfies $r\left(\mathcal{G} \cap S_{E_{0}}\right) \subseteq S_{E_{0}}$.

- The set $\mathcal{G} \cap S_{E_{0}} \cap\left(\mathcal{L}_{X_{L}}(h \circ \pi)\right)^{-1}(0)$ satisfies the condition containing Equation (4).

Then the restricted system

$$
H_{E_{0}}=\left(\mathcal{X}_{E_{0}}, \mathcal{F}_{E_{0}}, \mathcal{G}_{E_{0}}, \varphi_{E_{0}}, r_{E_{0}}\right):=\left(\mathcal{X} \cap S_{E_{0}}, \mathcal{F} \cap S_{E_{0}}, \mathcal{G} \cap S_{E_{0}}, \varphi_{E_{0}},\left.r\right|_{\mathcal{G} \cap S_{E_{0}}}\right)
$$

(where $\varphi_{E_{0}}$ is the restriction of $\varphi$ to $\operatorname{dom}(\varphi) \cap\left([0, \infty) \times S_{E_{0}}\right)$ ) is well-defined and satisfies Assumptions 1, 2, 3 , and 4 . Hence $H_{E_{0}}$ admits a complete Lyapunov function and $R\left(H_{E_{0}}\right)$ admits a Conley decomposition.

Proof. Conservation of energy [MR94, Prop. 7.3.1] implies that $E^{-1}\left(\left(-\infty, E_{0}\right]\right)$ is forward invariant under the flow of $X_{L}$, and so the first bulleted condition implies that $S_{E_{0}}$ is forward invariant for $H$; hence we may apply Prop. 3 to the restricted system $H_{E_{0}}$.

\section{EXAMPLES}

We first show in Ex. 4 that, even if an MHS satisfies all hypotheses of Theorems 1 and 2 except the trapping guard condition, the conclusions of both theorems can fail. We then proceed to give two examples motivated by toy models in classical mechanics. The first (Ex. 5, a special case of Cor. 1) illustrates a system to which Theorems 1 and 2 apply. The second (Ex. 6) illustrates a system to which they do not.

Example 4 (Failure in the absence of trapping guards). This example shows that the conclusions of both Theorems 1 and 2 can fail if Assumption 3 (the trapping guard hypothesis) is violated, even if Assumptions 1, 2, and 4 are satisfied. Define the MHS $H=(\mathcal{X}, \mathcal{F}, \mathcal{G}, \varphi, r)$ as follows. Let

$$
\mathcal{X}:=[-1,0] \cup[1,3] \subseteq \mathbb{R}, \quad \mathcal{G}:=\{0,2,3\}, \quad \mathcal{F}:=\mathcal{X} \backslash \mathcal{G} .
$$

We let $\varphi$ be generated by the vector field $-t \frac{\partial}{\partial t}$ on $[-1,0]$ and by the constant vector field $\frac{\partial}{\partial t}$ on $[1,3]$. We define $r: \mathcal{G} \rightarrow \mathcal{X}$ by $r(0)=1$ and $r(2)=r(3)=-1$. Clearly $H$ is deterministic and nonblocking.

It is easy to check that $R(H)=[-1,0] \cup[1,2]$, that $R(H)$ consists of a single chain equivalence class, and that every subset $J \subseteq \mathcal{X}$ satisfies $\omega(J)=\{0\}$. But $\{0\}$ is not forward invariant, so there are no nontrivial attracting-repelling pairs. It follows that the conclusions of Theorem 1 are violated.

\footnotetext{
${ }^{9}$ As opposed (presumably) to [AZGS06, Def. 1], we do not require $Q$ to be a manifold of fixed dimension, and we do not require that $h^{-1}(0)$ be a smooth manifold. We could have also required less smoothness of $L$ and $h$, but we do not bother with this here; the interested reader can refer to Prop. 3 for more refined smoothness assumptions.

${ }^{10}$ In [AZGS06], the reset map $r$ is given a rather specific definition, which generalizes the reset map of Ex. 5, but we do not require the use of this specific definition here.
} 


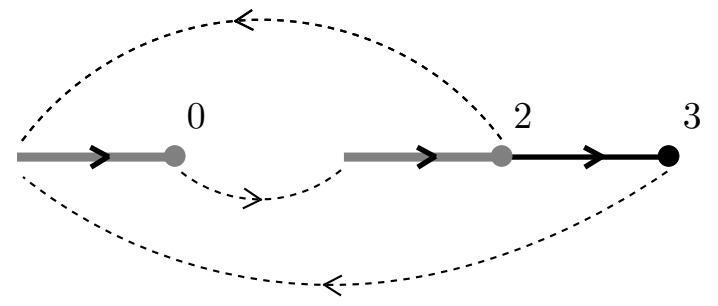

Figure 4. Depicted here is the MHS $H=(\mathcal{X}, \mathcal{F}, \mathcal{G}, \varphi, r)$ of Ex. 4. The chain recurrent set is the union of the two thick gray line segments and gray dots. For this example $\mathcal{X}=[-1,0] \cup[1,3] \subseteq \mathbb{R}, \mathcal{G}=\{0,2,3\}$, and $\mathcal{F}=\mathcal{X} \backslash \mathcal{G}$. The local semiflow $\varphi$ is generated by the vector field $-t \frac{\partial}{\partial t}$ on $[-1,0]$ and by the constant vector field $\frac{\partial}{\partial t}$ on $[1,3] . r$ is defined by $r(0)=1$ and $r(2)=r(3)=-1$. The MHS $H$ satisfies all hypotheses of Theorems 1 and 2 except for the trapping guard condition, and $H$ violates the conclusions of both of these theorems.
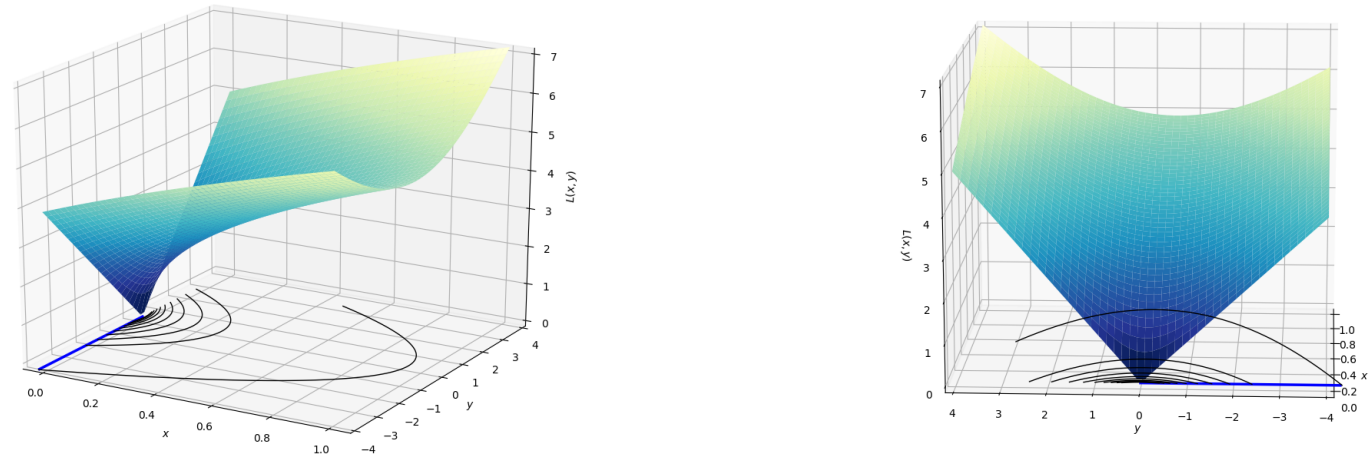

FiguRE 5. Depicted here are objects associated with the "bouncing ball" MHS $H=$ $(\mathcal{X}, \mathcal{F}, \mathcal{G}, \varphi, r)$ of Ex. 5 with coefficient of restitution $d=\frac{4}{5}$. $\mathcal{X}$ is the closed half plane $\{x \geq 0\}, \mathcal{F}$ is the interior $\{x>0\}$ of $\mathcal{X}$, and $\mathcal{G}$ is the ray $\{x=0, y \leq 0\}$ shown in blue. A portion of the unique execution in the hybrid system of Ex. 5 with initial condition $(x, y)=\left(\frac{1}{2}, 3\right)$ is shown in black; since $d<1$, this execution is Zeno. Shown above is the graph of the complete Lyapunov function $L$ given by Equation (6) with choice of constants $b=\frac{7}{5}, a=\frac{999}{1000} \cdot \frac{(1-d) g}{2 \sqrt{2} d} b$.

We further claim that no complete Lyapunov function $L: \mathcal{X} \rightarrow \mathbb{R}$ for $H$ exists, so that the conclusion of Theorem 2 is also violated. Indeed, suppose such an $L$ exists. By the continuity of $L$ and Condition 12.1 of Def. 12, we have $L(2)>L(3)$. By Condition 12.2, we have $L(3)>L(-1)$. Finally, Condition 12.3 implies that $L(-1)=L(2)$. This implies that $L(2)>L(3)>L(-1)=L(2)$, a contradiction.

We emphasize that $H$ satisfies all hypotheses of Theorems 1 and 2 except the trapping guard condition.

Example 5 (Bouncing ball). Let $g>0$ and $X$ be the complete analytic vector field on $\mathbb{R}^{2}$ given by

$$
X(x, y)=y \frac{\partial}{\partial x}-g \frac{\partial}{\partial y} .
$$

If we think of $x$ as the vertical position of a point particle with unit mass moving under the influence of gravity, and think of $y$ as its velocity, then the dynamics determined by $X$ are equivalent to those 
determined by Newton's second law of motion, $\ddot{x}=-g$. Letting $\Phi: \mathbb{R} \times \mathbb{R}^{2} \rightarrow \mathbb{R}^{2}$ be the analytic flow generated by $X$, we now construct an MHS which represents a "bouncing ball," and we show that this MHS satisfies all hypotheses of Theorems 1 and 2 after restriction to a compact forward invariant set.

Let $\mathcal{X}$ be the closed half plane $\mathcal{X}=\{x \geq 0\}$; we endow $\mathcal{X}$ with the metric induced by the Euclidean distance. Let $\mathcal{F}$ be the interior of $\mathcal{X}$, and define $\mathcal{G}:=\{(0, y) \mid y \leq 0\}$. Let $\varphi$ be the continuous semiflow given by the restriction of $\Phi$ to $(\Phi)^{-1}(\mathcal{F}) \cap(\mathcal{F} \times[0, \infty))$, and let the reset $r: \mathcal{G} \rightarrow \mathcal{X}$ be given by

$$
r(0, y):=(0,-d y),
$$

where $d \in[0,1]$ is the coefficient of restitution (which is related to the energy lost by the ball at impact). Clearly the MHS $H=(\mathcal{X}, \mathcal{F}, \mathcal{G}, \varphi, r)$ is deterministic (Assumption 1).

We now verify that $H$ satisfies the trapping guard condition (Assumption 3). We define the neighborhood $U$ of Def. 11 to be $U:=\mathcal{X}$. Integrating the vector field $X$ analytically yields

$$
\Phi^{t}(x, y)=\left(x+y t-\frac{g}{2} t^{2}, y-g t\right) .
$$

Setting the first component on the right hand side equal to 0 and solving the resulting quadratic equation, we find that the maximum flow time $\mu: \mathcal{X} \rightarrow[0,+\infty]$ (Equation 1) is given by

$$
\mu(x, y)=\frac{y+\sqrt{y^{2}+2 x g}}{g},
$$

which is clearly continuous. We define the continuous flow-induced retraction $\rho: \mathcal{X} \rightarrow \mathcal{G}$ by

$$
\rho(x, y):=\Phi^{\mu(x, y)}(x, y)=\left(0,-\sqrt{y^{2}+2 x g}\right) .
$$

Hence $\widehat{\varphi}:=\left.\Phi\right|_{\operatorname{cl}(\operatorname{dom}(\varphi)) \cap[0, \infty) \times \mathcal{X}}$ is a continuous extension of $\varphi$ to the closure of $\operatorname{dom}(\varphi)$ in $[0, \infty) \times \mathcal{X}$, as required in Def. 11 , and this extension is unique since $\mathbb{R}^{2}$ is Hausdorff.

The above shows that $H$ satisfies the trapping guard condition. To investigate Zeno executions, we compute the total time elapsed during the execution initialized at $(x, y)$ :

$$
\sum_{n=0}^{\infty} \mu \circ(r \circ \rho)^{\circ n}(x, y)=\sum_{n=0}^{\infty} \mu\left(0, d^{n} \sqrt{y^{2}+2 x g}\right)=\frac{2 \sqrt{y^{2}+2 x g}}{g} \sum_{n=0}^{\infty} d^{n} .
$$

Since the last sum is finite if and only if $d \in[0,1)$, it follows that (i) every maximal execution in $H$ initialized in $\mathcal{X} \backslash\{\mathbf{0}\}$ is Zeno if $0 \leq d<1$, and (ii) every maximal execution in $H$ initialized in $\mathcal{X} \backslash\{\mathbf{0}\}$ is infinite if $d=1$. (For any value of $d \in[0,1]$, the execution initialized at $\mathbf{0}$ is Zeno.) Hence $H$ satisfies Assumption 2.

The preceding shows that $H$ satisfies Assumptions 1, 2, and 3 but not the compactness Assumption 4 . However, for any finite $E_{0}>0$, the "energy" sublevel set $S_{E_{0}}:=\left\{(x, y) \in \mathcal{X} \mid \frac{1}{2} y^{2}+g x \leq E_{0}\right\}$ is compact and forward invariant for $H$. Therefore, the restricted system

$$
H_{E_{0}}=\left(\mathcal{X}_{E_{0}}, \mathcal{F}_{E_{0}}, \mathcal{G}_{E_{0}}, \varphi_{E_{0}}, r_{E_{0}}\right)=\left(\mathcal{X} \cap S_{E_{0}}, \mathcal{F} \cap S_{E_{0}}, \mathcal{G} \cap S_{E_{0}}, \varphi_{E_{0}},\left.r\right|_{\mathcal{G} \cap S_{E_{0}}}\right)
$$

(where $\varphi_{E_{0}}$ is the restriction of $\varphi$ to $\operatorname{dom}(\varphi) \cap\left([0, \infty) \times S_{E_{0}}\right)$ ) satisfies Assumptions 1, 2, 3, and 4 and hence all hypotheses of Theorems 1 and 2 (cf. Remark 9).

When the coefficient of restitution $d=1$, it is easy to see that the only attracting-repelling pairs for $H_{E_{0}}$ are the trivial pairs $\left(A, A^{*}\right)=\left(\mathcal{X}_{E_{0}}, \varnothing\right)$ and $\left(A, A^{*}\right)=\left(\varnothing, \mathcal{X}_{E_{0}}\right)$. According to Theorem 1 , the chain recurrent set $R\left(H_{E_{0}}\right)$ is therefore given by $R\left(H_{E_{0}}\right)=\mathcal{X}_{E_{0}}$. According to Theorem 2, $H_{E_{0}}$ has a complete Lyapunov function; since $R\left(H_{E_{0}}\right)=\mathcal{X}_{E_{0}}$ and since $\mathcal{X}_{E_{0}}$ is connected, the complete Lyapunov functions are precisely the constant real-valued functions on $\mathcal{X}_{E_{0}}$.

When the coefficient of restitution $d \in[0,1)$, it is easy to see that $H_{E_{0}}$ has a unique nontrivial attractingrepelling pair $\left(A, A^{*}\right)$ : the attracting set $A$ is the origin $\mathbf{0}$, and the dual repelling set $A^{*}$ is the empty set. According to Theorem 1, the chain recurrent set is therefore given by $R\left(H_{E_{0}}\right)=\{\mathbf{0}\}$. According to Theorem $2, H_{E_{0}}$ has a complete Lyapunov function. One family of such complete Lyapunov functions are given by a linear combination of the maximum flow time and the square root of the total energy,

$$
L(x, y)=a \mu(x, y)+b \sqrt{\frac{1}{2} y^{2}+g x}
$$


where $a, b>0$ are constants satisfying $\frac{1-d}{\sqrt{2}} b>\frac{2 d}{g} a$. The first term on the right strictly decreases along the continuous-time dynamics, while the second term is constant. On $\mathcal{G} \backslash\{\mathbf{0}\}$, the first term on the right strictly decreases upon applying the reset map, while the second term strictly increases; however, the inequality $\frac{1-d}{\sqrt{2}} b>\frac{2 d}{g} a$ ensures that

$$
\begin{aligned}
L(r(0, y))-L(0, y) & =a \cdot\left(\frac{2 d|y|}{g}-0\right)+b \cdot\left(\sqrt{\frac{1}{2} d^{2} y^{2}}-\sqrt{\frac{1}{2} y^{2}}\right) \\
& =\left(a \frac{2 d}{g}-b\left(\frac{1-d}{\sqrt{2}}\right)\right)|y|<0,
\end{aligned}
$$

as desired. Because $L$ does not depend on $E_{0}, L$ is also a complete Lyapunov function for the full system $H$.

In closing, we remark that this example is a special case of the hybrid Lagrangian systems considered in Cor. 1 with (in the notation of the corollary) $Q:=\mathbb{R}, L(x, y):=\frac{1}{2} y^{2}-g x, h(x):=x$, and $r(0, y):=(0,-d y)$. Since the restriction of $E(x, y)=\frac{1}{2} y^{2}+g x$ to $\mathcal{X}$ is a proper function, $S_{E_{0}}$ is compact for every $E_{0}>0$. In this example, $\mathcal{G} \cap S_{E_{0}} \cap\left(\mathcal{L}_{X} \psi\right)^{-1}(0)=\{\mathbf{0}\}$ is just the origin, and (4) is satisfied since $\mathcal{L}_{X_{L}}^{2}(h \circ \pi)=\ddot{x}=-g<0$ on all of $\mathcal{X}$. Thus, Cor. 1 directly implies that $H_{E_{0}}$ satisfies all hypotheses of Theorems 1 and 2, although the above "hands-on" verification seems instructive.

Example 6. In this example we consider a system which, while superficially similar to the bouncing ball of Ex. 5, does not satisfy the trapping guard condition (Assumption 3), so the hypotheses of Theorems 1 and 2 are not satisfied. Nevertheless, as we show below, this system does satisfy the conclusions of these two theorems. Thus, Theorems 1 and 2 could potentially be sharpened in future work.

We define the deterministic (Assumption 1) MHS $H=(\mathcal{X}, \mathcal{F}, \mathcal{G}, \varphi, r)$ to be given exactly as in Ex. 5 , except we replace the vector field $X$ of Ex. 5 with

$$
X(x, y)=y \frac{\partial}{\partial x}-x \frac{\partial}{\partial y} .
$$

Intuitively, $X$ is the vector field obtained from Newton's second law of motion where the gravitational force of Ex. 5 is replaced by a Hookean spring with unit stiffness and rest position $x=0$ (so $\ddot{x}=-x$ ).

A computation as in Ex. 5 verifies Assumption 2 since, in this example, every maximal execution initialized in $\mathcal{X} \backslash\{\mathbf{0}\}$ is infinite, while the execution initialized at $\mathbf{0}$ is Zeno. On $\mathbb{R}^{2} \backslash\{\mathbf{0}\}, X$ is given in polar coordinates $x=\rho \cos \theta, y=\rho \sin \theta$ as

$$
X(\rho, \theta)=-\frac{\partial}{\partial \theta} .
$$

Hence the maximum flow time $\mu: \mathcal{X} \rightarrow[0,+\infty]$ (Equation 1 ) is given by

$$
\left\{\begin{array}{ll}
\mu(\rho, \theta)=\theta+\frac{\pi}{2}, & \theta \in\left[-\frac{\pi}{2}, \frac{\pi}{2}\right] \text { and } \rho \neq 0 \\
\mu(\rho, \theta)=0, & \theta \in\left[-\frac{\pi}{2}, \frac{\pi}{2}\right] \text { and } \rho=0
\end{array},\right.
$$

which is discontinuous at the origin $\mathbf{0} \in \mathcal{G} \subseteq \mathcal{X}$, so $H$ does not satisfy the trapping guard condition (Assumption 3). Since also 0 belongs to every energy sublevel set $S_{E_{0}}:=\left\{(x, y) \mid \frac{1}{2} \rho^{2} \leq E_{0}\right\}$ for which $S_{E_{0}} \cap \mathcal{X} \neq \varnothing$, the restricted system

$$
H_{E_{0}}=\left(\mathcal{X}_{E_{0}}, \mathcal{F}_{E_{0}}, \mathcal{G}_{E_{0}}, \varphi_{E_{0}}, r_{E_{0}}\right)=\left(\mathcal{X} \cap S_{E_{0}}, \mathcal{F} \cap S_{E_{0}}, \mathcal{G} \cap S_{E_{0}}, \varphi_{E_{0}},\left.r\right|_{\mathcal{G} \cap S_{E_{0}}}\right)
$$

defined as in Ex. 5 does not satisfy the hypotheses of Theorems 1 and 2 for any value of $E_{0}$ such that $S_{E_{0}} \cap \mathcal{X} \neq \varnothing$.

However, in this example it is nevertheless easy to check that the conclusions of Theorem 1 and 2 still hold (for $H$, not merely $H_{E_{0}}$ ). When the coefficient of restitution $d=1, H$ has only the trivial attracting-repelling pairs, $R(H)=\mathcal{X}$, and any constant function on $\mathcal{X}$ is a complete Lyapunov function.

When $d \in[0,1)$, the only nontrivial attracting-repelling pair for $H$ is $(\{\mathbf{0}\}, \varnothing), R(H)=\{\mathbf{0}\}$, and one family of complete Lyapunov functions on $\mathcal{X}$ is given in polar coordinates by

$$
L(\rho, \theta)=a \rho \mu(\rho, \theta)+b \rho,
$$




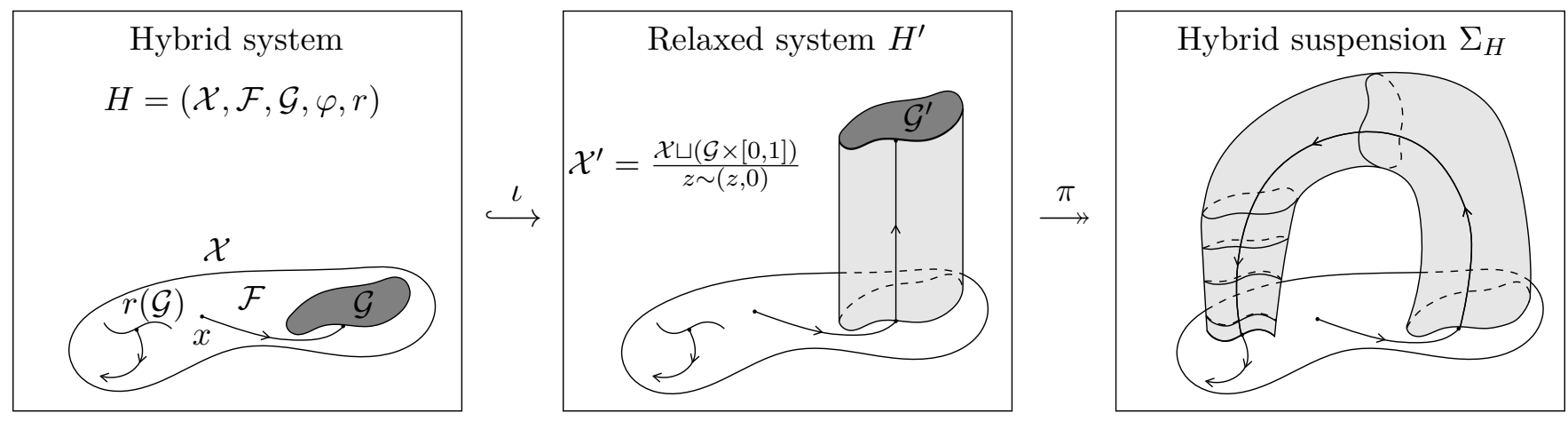

FiguRE 6. Illustration of the constructions in $\S 6.1$. Left: a THS $H=(\mathcal{X}, \mathcal{F}, \mathcal{G}, \varphi, r)$. Middle: its relaxed version $H^{\prime}=\left(\mathcal{X}^{\prime}, \mathcal{F}^{\prime}, \mathcal{G}^{\prime}, \varphi^{\prime}, r^{\prime}\right)$; see Def. 13. Right: the hybrid suspension $\left(\Sigma_{H}, \Phi_{H}\right)$ of $H$; see Def. 14 . We remark that $\left(\Sigma_{H}, \Phi_{H}\right)$ strictly generalizes the classical suspension of a discrete-time dynamical system [Sma67, BS02, p. 797, pp. 21-22]; indeed, if $\mathcal{X}=\mathcal{G}$ our construction reduces to the classical one (see Ex. 1, and see SM §A.4 and SM $\S \mathrm{B}$ for more details).

where $a, b>0$ satisfy $a<\frac{(1-d) b}{d \pi}$. To see that $L$ is indeed a complete Lyapunov function, note that $\rho$ is constant along $\varphi$ trajectories while $\mu$ strictly decreases, and $L$ decreases along resets from $\mathcal{G} \backslash\{\mathbf{0}\}$ since

$$
L(d \rho, \pi / 2)-L(\rho,-\pi / 2)=(a d \pi+b(d-1)) \rho<0 .
$$

\section{Proofs of the MAin Results}

This section culminates (in §6.3) with the proofs of Theorems 1 and 2. The earlier subsections develop the necessary tools.

Our development of these tools relies heavily on topologically "gluing" multiple topological spaces together and to themselves. The motivation for doing so - inspired by the "hybrifold" technique [SJSL00, SJLS05] - is to somehow reduce the study of a hybrid dynamical system to that of an associated "classical" continuous-time dynamical system for which comparatively well-developed theory already exists. "Gluing" is made precise using the concepts of quotient maps and quotient spaces from elementary point-set topology. A quotient map $\pi: X \rightarrow Y$ between topological spaces $X$ and $Y$ is a continuous surjective map having the property that $U \subseteq Y$ is open in $Y$ if and only if $\pi^{-1}(U)$ is open in $X$. Given an equivalence relation $\sim$ on a topological space $X$, we employ the standard notation $X / \sim$ for the topological quotient space of $X$ by $\sim$. Viewed as a set, $X / \sim$ is the set of all equivalence classes, but $X / \sim$ is also given a topology. If we denote by $\pi: X \rightarrow X / \sim$ the map sending points to their equivalence classes, the topology on $X / \sim$ is uniquely defined by requiring that $\pi$ be a quotient map. One thinks of $X / \sim$ as obtained from $X$ by "gluing" points $x, y \in X$ together if $x \sim y$. We refer readers wanting more details to the standard introductory textbook [Mun00, p. 137, p. 139]).

6.1. Suspension of a hybrid system. Given a THS $H=(\mathcal{X}, \mathcal{F}, \mathcal{G}, \varphi, r)$ satisfying Assumptions 1 , 2, and 3 , in this section we will construct two new systems: (i) a THS $H^{\prime}=\left(\mathcal{X}^{\prime}, \mathcal{F}^{\prime}, \mathcal{G}^{\prime}, \varphi^{\prime}, r^{\prime}\right)$ with $\mathcal{X}^{\prime}$ containing $\mathcal{X}$ as a proper subset which we term the relaxed hybrid system, and (ii) a continuous semiflow on a quotient $\Sigma_{H}$ of $H^{\prime}$ which we term the hybrid suspension of $H$. The basic ideas are contained in Fig. 6 . For the interested reader, SM $\S \mathrm{A} .4$ contains a comparison of $H^{\prime}$ and $\Sigma_{H}$ to related constructions appearing in the literature.

The relaxed system $H^{\prime}$ formalizes the idea of requiring that $H$ executions "wait" one time unit after impacting the guard before resetting. For us, the relaxed system is primarily a means to an end; it is an intermediate step in constructing the hybrid suspension, and it is also useful in analyzing some of the latter system's properties. We construct the relaxed system after the following two lemmas. The statement of Lemma 1 involves the maximum flow time $\mu: \mathcal{X} \rightarrow[0,+\infty]$ defined in (1); we defer the proof of Lemma 1 to SM $\S \mathrm{D}$. 
Lemma 1. Let $H=(\mathcal{X}, \mathcal{F}, \mathcal{G}, \varphi, r)$ be a THS satisfying Assumptions 1,2 , and 3 . Then $\mu: \mathcal{X} \rightarrow[0,+\infty]$ is continuous, the closure $\operatorname{cl}(\operatorname{dom}(\varphi))$ of $\operatorname{dom}(\varphi)$ in $[0, \infty) \times \mathcal{X}$ satisfies

$$
\operatorname{cl}(\operatorname{dom}(\varphi))=\{(t, x) \in[0, \infty) \times \operatorname{cl}(\mathcal{F}) \mid t \leq \mu(x)\},
$$

and $\varphi$ has a unique continuous extension $\widetilde{\varphi}$ defined on $\operatorname{cl}(\operatorname{dom}(\varphi))$ satisfying $\widetilde{\varphi}^{\mu(x)}(x) \in \mathcal{G}$ for all $x \in$ $\operatorname{cl}(\mathcal{F}) \cap \mu^{-1}([0, \infty))$.

Furthermore, $\widetilde{\varphi}$ satisfies the following conditions, with $t, s \in[0, \infty):(\mathrm{i}) \widetilde{\varphi}^{0}=\mathrm{id}_{\mathrm{cl}(\mathcal{F})}$, (ii) $(t+s, x) \in$ $\operatorname{cl}(\operatorname{dom}(\varphi)) \Longleftrightarrow \operatorname{both}(s, x) \in \operatorname{cl}(\operatorname{dom}(\varphi))$ and $\left(t, \widetilde{\varphi}^{s}(x)\right) \in \operatorname{cl}(\operatorname{dom}(\varphi))$, and (iii) for all $(t+s, x) \in$ $\operatorname{cl}(\operatorname{dom}(\varphi)), \widetilde{\varphi}^{t+s}(x)=\widetilde{\varphi}^{t}\left(\widetilde{\varphi}^{s}(x)\right)$.

Lemma 2. Let $X$ be a metrizable space and $A \subseteq X$ a closed subset. Let $f: A \rightarrow X$ be a continuous and closed map satisfying $f(A) \cap A=\varnothing$. Define an equivalence relation $\sim$ on $X$ by identifying each $a \in A$ with $f(a) \in X$. Then the quotient map $\pi: X \rightarrow X / \sim$ is closed. If additionally $f$ has compact fibers (i.e., $f^{-1}(x)$ is compact for all $\left.x \in X\right)$, then the quotient space $X / \sim$ is metrizable.

Remark 11. If $A$ is compact and $f$ is continuous, then $f$ is automatically closed and proper; hence also $f^{-1}(x)$ is compact for all $x \in X$.

Proof. We first show that $\pi$ is closed. Letting $C \subseteq X$ be any closed subset, we compute

$$
\pi^{-1}(\pi(C))=C \cup f^{-1}(C) \cup f(C \cap A)
$$

since $f(A) \cap A=\varnothing$. The first set on the right is closed by definition, and the second term is closed since $A$ is closed and $f$ is continuous. The third term is closed since $f$ is a closed map. Hence $\pi^{-1}(\pi(C))$ is closed, and this in turn implies that $\pi(C)$ is closed by the definition of the quotient topology. Hence $\pi$ is closed.

We now prove that $X / \sim$ is metrizable under the additional assumption that $f$ has compact fibers. A theorem of Stone [Sto56, Thm 1] implies that, if $\pi$ is closed, then $X / \sim$ is metrizable if and only if $\pi^{-1}(\pi(x))$ has compact boundary for all $x \in X$. Therefore, it suffices to show that $\pi^{-1}(\pi(x))$ has compact boundary for all $x$.

Fix $x \in X$ and substitute $C=\{x\}$ in (8) to obtain

$$
\pi^{-1}(\pi(x))=\{x\} \cup f^{-1}(x) \cup f(\{x\} \cap A) .
$$

The first and third terms on the right are compact because they are either singletons or empty. The second term on the right is compact by our assumption that $f$ has compact fibers. Hence $\pi^{-1}(\pi(x))$ is compact, and this in turn implies that $\pi^{-1}(\pi(x))$ has compact boundary. This completes the proof.

The following definition uses the maximum flow time $\mu: \mathcal{X} \rightarrow[0,+\infty]$ defined in (1). As mentioned in $\S 1.1$ and 1.2, a version of the following definition appears in [JELS99]; see §A.4.2 for more details.

Definition 13 (Relaxed hybrid system). Let $H=(\mathcal{X}, \mathcal{F}, \mathcal{G}, \varphi, r)$ be a THS satisfying Assumptions 1, 2, and 3. We define the relaxed hybrid system $H^{\prime}=\left(\mathcal{X}^{\prime}, \mathcal{F}^{\prime}, \mathcal{G}^{\prime}, \varphi^{\prime}, r^{\prime}\right)$ associated to $H$ as follows. Let $\asymp$ be the equivalence relation on $Y:=\mathcal{X} \sqcup(\mathcal{G} \times[0,1])$ which identifies $\mathcal{G}$ with $\mathcal{G} \times\{0\}, \pi_{0}: Y \rightarrow Y / \asymp$ be the corresponding quotient map, and $\pi_{1}:[0, \infty) \times Y \rightarrow[0, \infty) \times(Y / \asymp)$ be the quotient map $\pi_{1}:=\operatorname{id}_{[0, \infty)} \times \pi_{0} \cdot{ }^{11}$ Let $U \supseteq \mathcal{G}$ be the domain of a flow-induced retraction as in Def. 11, and let $\widetilde{\varphi}$ be the unique continuous extension of $\varphi$ to the closure $\operatorname{cl}(\operatorname{dom}(\varphi))$ of $\operatorname{dom}(\varphi)$ in $\mathcal{X}$ ensured by Lemma 1 . We define the spaces

$$
\begin{aligned}
\mathcal{X}^{\prime} & :=\pi_{0}(\mathcal{X} \sqcup(\mathcal{G} \times[0,1])) \quad \mathcal{G}^{\prime}:=\pi_{0}(\mathcal{G} \times\{1\}) \quad \mathcal{F}^{\prime}:=\pi_{0}(\mathcal{X} \sqcup(\mathcal{G} \times[0,1))) \\
\operatorname{dom}\left(\varphi^{\prime}\right) & :=\pi_{1}\left(\{(t, x) \mid 0 \leq t<\mu(x)+1\} \sqcup \bigcup_{t \in[0,1)}\{t\} \times(\mathcal{G} \times[0,1-t))\right),
\end{aligned}
$$

\footnotetext{
${ }^{11}$ That $\pi_{1}$ is a quotient map (where $[0, \infty) \times Y$ has the product topology) follows since (i) $\pi_{0}$ is a quotient map, (ii) [0, $\left.\infty\right)$ is a locally compact Hausdorff space, and (iii) the product of a quotient map and the identity map of a locally compact Hausdorff space is always a quotient map [Lee10, Lem 4.72].
} 
where $\mathcal{X}^{\prime}$ has the quotient topology, and maps $r^{\prime}: \mathcal{G}^{\prime} \rightarrow \mathcal{X}^{\prime}, \varphi^{\prime}: \operatorname{dom}\left(\varphi^{\prime}\right) \rightarrow \mathcal{F}^{\prime}$ via

$$
\begin{aligned}
r^{\prime}\left(\pi_{0}(z, 1)\right) & :=\pi_{0}(r(z)), \quad z \in \mathcal{G} \\
\varphi^{\prime}\left(t, \pi_{0}(x)\right) & = \begin{cases}\pi_{0}\left(\widetilde{\varphi}^{t}(x)\right), & (t, x) \in \operatorname{cl}(\operatorname{dom}(\varphi)) \\
\pi_{0}\left(\widetilde{\varphi}^{\mu(x)}(x), t-\mu(x)\right), & x \in \operatorname{cl}(\mathcal{F}), t \in[\mu(x), \mu(x)+1) . \\
\pi_{0}(z, s+t), & x=(z, s) \in \mathcal{G} \times[0,1-t)\end{cases}
\end{aligned}
$$

Proposition 4. Let $H=(\mathcal{X}, \mathcal{F}, \mathcal{G}, \varphi, r)$ be a THS satisfying Assumptions 1,2 , and 3 . Then the relaxed hybrid system $H^{\prime}=\left(\mathcal{X}^{\prime}, \mathcal{F}^{\prime}, \mathcal{G}^{\prime}, \varphi^{\prime}, r^{\prime}\right)$ associated to $H$ is also a THS satisfying Assumptions 1,2 , and 3. Furthermore, $H^{\prime}$ is nonblocking.

If $H$ satisfies Assumption 4, then so does $H^{\prime}$. If $\mathcal{X}$ is metrizable and $r$ is a closed map with compact fibers, then $\mathcal{X}^{\prime}$ is metrizable. In particular, if $\mathcal{X}$ is metrizable and compact, then so is $\mathcal{X}^{\prime}$.

Remark 12 (Relaxation converts Zeno executions to infinite executions). The nonblocking statement of Prop. 4 shows that the original motivation for relaxation - elimination of Zeno behavior [JELS99] — still holds in our setting.

Proof. By the definition of the disjoint union and quotient topologies, $\mathcal{G}^{\prime}$ is closed in $\mathcal{X}^{\prime}, \mathcal{F}^{\prime}$ is open in $\mathcal{X}^{\prime}$, and $\operatorname{dom}\left(\varphi^{\prime}\right)$ is open in $[0, \infty) \times \mathcal{X}^{\prime}$. Since $r^{\prime}$ can be written as a composition of continuous functions, $r^{\prime}$ is continuous. Lemma 1 implies that the three functions defining $\varphi^{\prime}$ agree on the intersections of their domains; since each such domain is closed in $\operatorname{dom}\left(\varphi^{\prime}\right)$, the pasting lemma of point-set topology [Mun00, Thm 18.3] implies that $\varphi^{\prime}$ is continuous. From the properties of $\widetilde{\varphi}$ established in Lemma 1, it is clear that $\varphi^{\prime}$ is also a local semiflow. Hence $H^{\prime}$ is a THS, and $H^{\prime}$ is deterministic (Assumption 1) since $\mathcal{G}^{\prime} \cap \mathcal{F}^{\prime}=\varnothing$. Furthermore, $\mathcal{G}^{\prime}$ is clearly a trapping guard (Assumption 3). Since $H$ satisfies Assumption 2 and since every maximal $H^{\prime}$ execution contains every arc of some maximal $H$ execution, $H^{\prime}$ also satisfies Assumption 2. However, $H^{\prime}$ has no Zeno executions because all but possibly one resets of a maximal $H^{\prime}$ execution are preceded by an arc defined on an interval of length greater than one.

If $\mathcal{X}$ is compact (Assumption 4), then so is $\mathcal{G}$ and hence also $\mathcal{G} \times[0,1]$, so $\mathcal{X} \sqcup(\mathcal{G} \times[0,1]$ ) and (by continuity of $\left.\pi_{0}\right) \mathcal{X}^{\prime}$ are therefore compact. If $r$ is a closed map with compact fibers, then the same is true of $r^{\prime}$, and $r^{\prime}\left(\mathcal{G}^{\prime}\right) \cap \mathcal{G}^{\prime}=\varnothing$ by construction; thus if $\mathcal{X}$ is also metrizable, Lemma 2 implies that $\mathcal{X}^{\prime}$ is metrizable. By Remark 11, these conditions on $r$ are automatically satisfied if $\mathcal{X}$ is compact. This completes the proof.

For later use we record the following result which implies [Lee10, Ex. 2.29] that the "obvious embedding" $\iota: \mathcal{X} \rightarrow \mathcal{X}^{\prime}$ defined by $\iota:=\pi_{0} \mid \mathcal{X}$ is indeed a topological embedding (a homeomorphism onto its image).

Lemma 3. The quotient map $\pi_{0}: \mathcal{X} \sqcup(\mathcal{G} \times[0,1]) \rightarrow \mathcal{X}^{\prime}$ is closed.

Proof. Let $j: \mathcal{G} \rightarrow \mathcal{G} \times\{0\}$ be the obvious identification and $C \subseteq \mathcal{X} \sqcup(\mathcal{G} \times[0,1])$ be closed. Then, since $\mathcal{G}$ is closed in $\mathcal{X}$ and $j$ is a homeomorphism,

$$
\pi_{0}^{-1}\left(\pi_{0}(C)\right)=C \cup j^{-1}(C) \cup j(C \cap \mathcal{G})
$$

is closed by the definition of the disjoint union topology. By the definition of the quotient topology, $\pi_{0}(C)$ is therefore closed.

Using the relaxed hybrid system associated to $H=(\mathcal{X}, \mathcal{F}, \mathcal{G}, \varphi, r)$, we now define the hybrid suspension $\left(\Sigma_{H}, \Phi_{H}\right)$ of $H$. As mentioned in $\S 1.1$ and 1.2 , versions of the hybrid suspension have appeared in $\left[\mathrm{AS05}, \mathrm{BGV}^{+} 15\right]$ under different names; see $\S \mathrm{A} .4 .3$ for more details. We choose to call this space a "suspension" because it generalizes the classical suspension of a discrete-time dynamical system [Sma67, BS02, p. 797, pp. 21-22]; indeed, if $\mathcal{X}=\mathcal{G}$ our construction reduces to the classical one (see SM $\S \mathrm{B}$ for details). We will later see that the dynamics of $\left(\Sigma_{H}, \Phi_{H}\right)$ are particularly compatible with those of $H$ (Prop. 6, 7, 8). (Contrast this with the (generalized) hybrifold semiflow appearing in the literature and discussed in detail in SM §A.4.1; see also Remarks 15 and 17.)

Definition 14 (Hybrid suspension). Let $H=(\mathcal{X}, \mathcal{F}, \mathcal{G}, \varphi, r)$ be a THS satisfying Assumptions 1, 2 , and 3. Let $H^{\prime}=\left(\mathcal{X}^{\prime}, \mathcal{F}^{\prime}, \mathcal{G}^{\prime}, \varphi^{\prime}, r^{\prime}\right)$ be the relaxed hybrid system of Def. 13 . Let $\sim$ be the equivalence relation on 
$\mathcal{X}^{\prime}$ which identifies each $z^{\prime} \in \mathcal{G}^{\prime} \subseteq \mathcal{X}^{\prime}$ with $r^{\prime}\left(z^{\prime}\right) \in \mathcal{X}^{\prime}$. We define the topological space $\Sigma_{H}:=\mathcal{X}^{\prime} / \sim$ and let $\pi: \mathcal{X}^{\prime} \rightarrow \Sigma_{H}$ be the quotient map. Since $H^{\prime}$ is nonblocking, we may define $\Phi_{H}:[0, \infty) \times \Sigma_{H} \rightarrow \Sigma_{H}$ by declaring $\Phi_{H}^{t}\left(\pi\left(x^{\prime}\right)\right)$ to be the unique element of the singleton $\pi\left(\chi_{x^{\prime}}(t)\right)$ for $x^{\prime} \in \mathcal{X}^{\prime}$ and $t \geq 0$. We define $\left(\Sigma_{H}, \Phi_{H}\right)$ to be the hybrid suspension and suspension semiflow of $H$. For brevity, we sometimes simply refer to the pair $\left(\Sigma_{H}, \Phi_{H}\right)$ as the hybrid suspension.

The following proposition justifies the suspension semiflow nomenclature.

Proposition 5. Let $H=(\mathcal{X}, \mathcal{F}, \mathcal{G}, \varphi, r)$ be a THS satisfying Assumptions 1, 2, and 3. Let $\left(\Sigma_{H}, \Phi_{H}\right)$ be the hybrid suspension. Then $\Phi_{H}:[0, \infty) \times \Sigma_{H} \rightarrow \Sigma_{H}$ is a continuous semiflow.

If $H$ satisfies Assumption 4, then $\Sigma_{H}$ is compact. If $\mathcal{X}$ is metrizable and $r$ is a closed map with compact fibers, then $\Sigma_{H}$ is metrizable. In particular, if $\mathcal{X}$ is metrizable and compact, then so is $\Sigma_{H}$.

Proof. Letting $\chi_{x}$ denote the unique $H^{\prime}$ execution through $x \in I^{\prime}$ and using the notation $\chi_{x}(t)$ of Def. 2, we may view $(t, x) \mapsto \chi_{x}(t)$ as a set-valued map (SVM) $\chi$. Since Prop. 4 implies that $H^{\prime}$ is nonblocking, the SVM $\chi:[0, \infty) \times \mathcal{X}^{\prime} \rightarrow 2^{\mathcal{X}^{\prime}}$ has only nonempty values. Prop. 4 also implies that $H^{\prime}=\left(\mathcal{X}^{\prime}, \mathcal{F}^{\prime}, \mathcal{G}^{\prime}, \varphi^{\prime}, r^{\prime}\right)$ satisfies Assumptions 1, 2, and 3, so Lemma 1 implies that $\varphi^{\prime}$ has a unique continuous extension $\widetilde{\varphi}^{\prime}$ defined on $\operatorname{cl}\left(\operatorname{dom}\left(\varphi^{\prime}\right)\right)$. This and continuity of $r^{\prime}$ in turn imply that $\chi$ is upper semicontinuous (USC) [AC84, p. 40, Def. 1]. Since (i) $\pi$ is continuous, (ii) the composition of USC SVMs is USC [AC84, p. 41, Prop. 1], and (iii) singleton-valued USC SVMs are equivalent to continuous (single-valued) maps, $\pi \circ \chi$ may be identified with a continuous map $[0, \infty) \times \mathcal{X}^{\prime} \rightarrow \Sigma_{H}$. Since $\operatorname{id}_{[0, \infty)} \times \pi$ is a quotient map (cf. Footnote 11) and $\Phi_{H} \circ\left(\operatorname{id}_{[0, \infty)} \times \pi\right)=\pi \circ \chi$, the universal property of the quotient topology [Lee10, Thm 3.70] implies that $\Phi_{H}$ is continuous. Since $\varphi^{\prime}$ is a local semiflow, it is clear from the definition of $\chi$ that $\chi_{x}(t+s)=\chi_{y}(t)$ for any $y \in \chi_{x}(s)$, so $\Phi_{H}^{t+s}(\pi(x))=\pi\left(\chi_{y}(t)\right)=\Phi_{H}^{t}(\pi(y))=\Phi_{H}^{t}\left(\Phi_{H}^{s}(\pi(x))\right)$ and hence $\Phi_{H}$ is a semiflow.

If $\mathcal{X}$ is compact ( $H$ satisfies Assumption 4 ), then so is $\mathcal{X}^{\prime}$ by Prop. 4, and therefore $\Sigma_{H}=\pi\left(\mathcal{X}^{\prime}\right)$ is compact. if $r$ is a closed map with compact fibers, then the same is true of $r^{\prime}$, and since also $r^{\prime}\left(\mathcal{G}^{\prime}\right) \cap \mathcal{G}^{\prime}=\varnothing$, two applications of Lemma 2 imply that $\mathcal{X}^{\prime}$ and $\Sigma_{H}$ are metrizable. By Remark 11, these conditions on $r$ are automatically satisfied if the guard if $\mathcal{X}$ is compact. This completes the proof.

Remark 13 (Further motivation for the trapping guard condition.). Let $H=(\mathcal{X}, \mathcal{F}, \mathcal{G}, \varphi, r)$ be a THS satisfying Assumptions 1 and 2. Under the additional assumption that $H$ satisfies the trapping guard condition (Assumption 3), in Def. 13 and 14 we defined the spaces $\mathcal{X}^{\prime}, \Sigma_{H}$ and maps $\pi_{0}, \iota$, and $\pi$. We also constructed the suspension semiflow $\Phi_{H}:[0, \infty) \times \Sigma_{H} \rightarrow \Sigma_{H}$ and showed that $\Phi_{H}$ is continuous. It is immediate from the definitions that $\Phi_{H}$ satisfies the following two properties.

1. $\Phi_{H}^{t}\left(\pi \circ \pi_{0}(z, s)\right)=\pi \circ \pi_{0}(z, t+s)$ for all $z \in \mathcal{G}, s \in[0,1]$, and $t \in[0,1-s]$.

2. For all $(t, x) \in \operatorname{dom}(\varphi), \pi \circ \iota\left(\varphi^{t}(x)\right)=\Phi_{H}^{t}(\pi \circ \iota(x))$.

While for convenience of exposition we only defined the quantities $\mathcal{X}^{\prime}, \Sigma_{H}, \pi_{0}, \iota$, and $\pi$ under Assumptions 1, 2, and 3 (in particular, under Assumption 3), their definitions make sense (verbatim) for any THS. Thus, for an arbitrary THS $H$, it makes sense to ask the following question: under what circumstances does there exist a well-defined "suspension semiflow" $\Phi$ on $\Sigma_{H}$ for $H$ in the sense that $\Phi$ satisfies Conditions 1 and 2 (stated above for $\Phi_{H}$ )?

In $\mathrm{SM} \S \mathrm{C}$ we prove (Cor. 5) that, if $H=(\mathcal{X}, \mathcal{F}, \mathcal{G}, \varphi, r)$ is a THS satisfying Assumptions 1 and 2 with Hausdorff state space $\mathcal{X}$, then there exists a continuous suspension semiflow $\Phi:[0, \infty) \times \Sigma_{H} \rightarrow \Sigma_{H}$ in the above sense if and only if $H$ satisfies the trapping guard condition (Assumption 3). This further motivates the trapping guard condition.

Prop. 6 below relates hybrid $\omega$-limit sets (Def. 8) to those of the hybrid suspension semiflow, and can be viewed as motivation for the definition of hybrid $\omega$-limit sets.

Proposition 6. Let $H=(\mathcal{X}, \mathcal{F}, \mathcal{G}, \varphi, r)$ be a THS satisfying Assumptions 1, 2, and 3 and with $r: \mathcal{G} \rightarrow \mathcal{X}$ a closed map. Let $H^{\prime}=\left(\mathcal{X}^{\prime}, \mathcal{F}^{\prime}, \mathcal{G}^{\prime}, \varphi^{\prime}, r^{\prime}\right)$ be the relaxed hybrid system of Def. $13, \pi_{0}: \mathcal{X} \sqcup(\mathcal{G} \times[0,1]) \rightarrow \mathcal{X}^{\prime}$ be the quotient of Def. 13, $\iota: \mathcal{X} \rightarrow \mathcal{X}^{\prime}$ be the embedding $\left.\pi_{0}\right|_{\mathcal{X}}$, and $\left(\Sigma_{H}, \Phi_{H}\right)$ be the hybrid suspension of Def. 14 with quotient $\pi: \mathcal{X}^{\prime} \rightarrow \Sigma_{H}$. Let $\rho_{0}: \pi_{0}(\mathcal{G} \times[0,1]) \rightarrow \mathcal{G}$ be the composition of the straight-line retraction $\pi_{0}(\mathcal{G} \times[0,1]) \rightarrow \iota(\mathcal{G})$ with the identification $\iota(\mathcal{G}) \approx \mathcal{G}$. For all $B \subseteq \Sigma_{H}$, define

$$
\mathcal{G}_{B}:=\rho_{0}\left(\pi^{-1}(B) \cap \pi_{0}(\mathcal{G} \times[0,1])\right) .
$$


Then for all $B \subseteq \Sigma_{H}$ :

$$
(\pi \circ \iota)^{-1}(\omega(B))=\omega\left((\pi \circ \iota)^{-1}(B)\right) \cup \omega\left(\mathcal{G}_{B}\right)
$$

In particular, for all $A \subseteq \mathcal{X}$ :

$$
\omega(A)=(\pi \circ \iota)^{-1}(\omega(\pi \circ \iota(A))) .
$$

Remark 14. If $\mathcal{G}$ is compact and $\mathcal{X}$ is Hausdorff, then $r$ is automatically a closed map. In particular, the proposition holds if $H$ satisfies the hypotheses of Theorems 1 and 2 (i.e., if $H$ is an MHS satisfying Assumptions 1, 2, 3, and 4.)

Remark 15. The statement analogous to Prop. 6 obtained by replacing the hybrid suspension with the semiflow on the (generalized) hybrifold $M_{H}$ (defined in SM §A.4.1) is false. For example, consider a THS $H$ with $\mathcal{X}=\mathcal{G}=\{0,1\}, r(\mathcal{G})=\{0\}$, and $\mathcal{F}=\varnothing$ (cf. Ex. 1). Then $M_{H}$ is a singleton $\{*\}$. Letting $\pi_{M_{H}}: \mathcal{X} \rightarrow M_{H}$ be the quotient defined in SM $\S$ A.4.1 and $j \in\{0,1\}$, we compute

$$
\omega(j)=\{0\} \neq\{0,1\}=\pi_{M_{H}}^{-1}(\{*\})=\pi_{M_{H}}^{-1}\left(\omega\left(\pi_{M_{H}}(j)\right)\right),
$$

so the analogue of (10) is false for the (generalized) hybrifold semiflow. The same example shows that the statement analogous to Prop. 7 below for the (generalized) hybrifold semiflow is also false.

Proof of Prop. 6. For purposes of readability, for this proof we define $f:=\pi \circ \iota$ and $g:=\left.\left(\pi \circ \pi_{0}\right)\right|_{\mathcal{G} \times[0,1]}$, and we introduce the following notation for $T>0$ and subsets $A \subseteq \mathcal{X}, B \subseteq \Sigma_{H}$ :

$$
R_{A, T}:=\bigcup_{x \in A} \bigcup_{(N, \tau, \gamma) \in \mathcal{E}_{H}(x)}\left\{\gamma_{j}(t) \mid j+t \geq T\right\}, \quad S_{B, T}:=\Phi_{H}^{[T, \infty)}(B) .
$$

In the definition of $R_{A, T}$, note that each $\mathcal{E}_{H}(x)$ contains only a single maximal execution since we assume that $H$ is deterministic. For later use we note that $f$ is a closed map since it is the composition of (i) $\pi$, which is a closed map by Lemma 2, and (ii) $\iota$, which is a closed map since, for any closed $C \subseteq \mathcal{X}$, $\pi_{0}^{-1}(\iota(C))=C \sqcup((C \cap \mathcal{G}) \times\{0\})$ is closed in $(\mathcal{X} \sqcup \mathcal{G} \times[0,1])$. Furthermore, $g$ is a closed map by composition and restriction since (i) $\pi$ is closed, (ii) $\pi_{0}$ is closed by Lemma 3 , and (iii) $\mathcal{G} \times[0,1]$ is closed in $\mathcal{X} \sqcup(\mathcal{G} \times[0,1])$.

In order to prove the proposition, we first need to establish two facts: for any $B \subseteq \Sigma_{H}$,

$$
\omega(B)=(\omega(B \cap f(\mathcal{X}))) \cup \omega\left(f\left(\mathcal{G}_{B}\right)\right),
$$

and

$$
f(\mathcal{X}) \cap \bigcap_{T>0} \operatorname{cl}\left(S_{B, T}\right)=\bigcap_{T>0} \operatorname{cl}\left(f(\mathcal{X}) \cap S_{B, T}\right) .
$$

We begin by establishing (11). First note that, for all $T \geq 0$, the definitions of $\mathcal{G}_{B}$ and $\Phi_{H}$ immediately imply that

$$
\Phi_{H}^{[1+T, \infty)}\left(f\left(\mathcal{G}_{B}\right)\right) \subseteq \Phi_{H}^{[T, \infty)}(B \backslash f(\mathcal{X})) \subseteq \Phi_{H}^{[T, \infty)}\left(f\left(\mathcal{G}_{B}\right)\right)
$$

Equation (13) and the definition of $\omega$-limit sets imply that $\omega\left(f\left(\mathcal{G}_{B}\right)\right)=\omega(B \backslash f(\mathcal{X}))$. Since the $\omega$-limit set of a finite union is equal to the union of the $\omega$-limit $\operatorname{sets}^{12}, \omega(B)=\omega(B \cap f(\mathcal{X})) \cup \omega(B \backslash f(\mathcal{X}))$, from which (11) now follows.

We now establish (12) for fixed $B \subseteq \Sigma_{H}$; for readability, we define $C_{B}$ to be the set on the left side of $(12)$ and $D_{B}$ to be the set on the right. Since $f$ is a closed map, $f(\mathcal{X})$ is closed in $\Sigma_{H}$, so it is immediate from general topology that $D_{B} \subseteq C_{B}$. Hence we need only prove that $C_{B} \subseteq D_{B}$. To obtain a contradiction, suppose that this is not the case, so that there exists $x \in C_{B} \backslash D_{B}$. Clearly we must have $x \notin \operatorname{int}(f(\mathcal{X}))$, so $x$ belongs to the boundary $g(\mathcal{G} \times\{0,1\})$ of $f(\mathcal{X})$. Since $x \notin D_{B}$, there exists $T_{0}>0$ and a neighborhood $V_{x}$ of $x$ such that $V_{x} \cap f(\mathcal{X}) \cap S_{B, T_{0}}=\varnothing$. Since the family $\left(S_{B, T}\right)_{T>0}$ decreases in $T$, this implies that

$$
V_{x} \cap f(\mathcal{X}) \cap S_{B, T}=\varnothing \text { for all } T \geq T_{0} .
$$

\footnotetext{
${ }^{12}$ Proof: since the closure of a finite union is the union of the closures, we compute $\omega(U \cup V)=\bigcap_{T>0} \operatorname{cl}\left(\Phi_{H}^{[T, \infty)}(U \cup V)\right)=$ $\bigcap_{T>0} \operatorname{cl}\left(\Phi_{H}^{[T, \infty)}(U)\right) \cup \operatorname{cl}\left(\Phi_{H}^{[T, \infty)}(V)\right)=\omega(U) \cup \omega(V)$ for any sets $U, V$. (This result is stated in [Con78, II.4.1.C] for the special case of a flow.) Repeating the same proof mutatis mutandis shows that hybrid $\omega$-limit sets also possess this finite-union property.
} 
Let $Y:=\mathcal{G} \times([0,1 / 4) \cup(3 / 4,1])$ and $\tilde{\nu}: Y \rightarrow \mathcal{G} \times\{0,1\}$ be the straight-line retraction. Since $g$ is a closed map, $g$ is a quotient map onto its image. Furthermore, since $Y$ is an open $g$-saturated ${ }^{13}$ subset of $\mathcal{G} \times[0,1]$, it follows that (i) $g(Y)$ is open relative to $g(\mathcal{G} \times[0,1])$ and (ii) $\left.g\right|_{Y}: Y \rightarrow g(Y)$ is also a quotient map [Lee10, Prop. 3.62.d]. We also note that, if $g(z, t)=g\left(z^{\prime}, t^{\prime}\right)$ for some distinct points $(z, t),\left(z^{\prime}, t^{\prime}\right) \in Y$, then necessarily $(z, t),\left(z^{\prime}, t^{\prime}\right) \in \mathcal{G} \times\{0,1\}$ and hence $(g \circ \tilde{\nu})(z, t)=g(z, t)=g\left(z^{\prime}, t^{\prime}\right)=(g \circ \tilde{\nu})\left(z^{\prime}, t^{\prime}\right)$ since $\left.\tilde{\nu}\right|_{\mathcal{G} \times\{0,1\}}=\operatorname{id}_{\mathcal{G} \times\{0,1\}}$. Thus, by the universal property of the quotient topology [Lee10, Thm 3.70], the map $g \circ \tilde{\nu}$ descends to a continuous trajectory-preserving retraction $\nu: g(U) \rightarrow g(\mathcal{G} \times\{0,1\})$. We now define a new set $U_{x}:=V_{x} \cap \nu^{-1}\left(V_{x} \cap g(\mathcal{G} \times\{0,1\})\right)$. Since $U_{x}$ is open relative to $g(Y)$ which is in turn open relative to $g(\mathcal{G} \times[0,1]), U_{x}$ is also open relative to $g(\mathcal{G} \times[0,1])$ and hence the set $U_{x} \cup f(\mathcal{X})$ is a neighborhood of $x$. Thus, since $x \in C_{B}$, for every $T \geq T_{0}$ there exists $y_{T} \in S_{B, T} \cap\left(U_{x} \cup f(\mathcal{X})\right) \cap V_{x}=S_{B, T} \cap U_{x}$. Thus, by the definitions of the suspension semiflow, $\nu$, and $U_{x}$, we have $\nu\left(y_{T}\right) \in V_{x} \cap f(\mathcal{X}) \cap S_{B, T-\frac{1}{4}}$ for any $T \geq T_{0}+\frac{1}{4}$, contradicting (14). This establishes (12).

Armed with (11) and (12), we now proceed to prove the proposition. Since $B \cap f(\mathcal{X})$ and $f\left(\mathcal{G}_{B}\right)$ are subsets of $f(\mathcal{X})$, it is immediate from the definitions that, for all $T \geq 0$,

$$
f(\mathcal{X}) \cap S_{B \cap f(\mathcal{X}), T}=f\left(R_{f^{-1}(B), T}\right), \quad f(\mathcal{X}) \cap S_{f\left(\mathcal{G}_{B}\right), T}=f\left(R_{\mathcal{G}_{B}, T}\right) .
$$

The following computation, to be justified after, proves (9).

$$
\begin{aligned}
f^{-1}(\omega(B)) & =f^{-1}\left(\omega(B \cap f(\mathcal{X})) \cup \omega\left(f\left(\mathcal{G}_{B}\right)\right)\right) \\
& =f^{-1}\left(\bigcap_{T>0} \operatorname{cl}\left(S_{B \cap f(\mathcal{X}), T}\right) \cup \bigcap_{T>0} \operatorname{cl}\left(S_{f\left(\mathcal{G}_{B}\right), T}\right)\right) \\
& =f^{-1}\left(\bigcap_{T>0} f(\mathcal{X}) \cap \operatorname{cl}\left(S_{B \cap f(\mathcal{X}), T}\right) \cup \bigcap_{T>0} f(\mathcal{X}) \cap \operatorname{cl}\left(S_{f\left(\mathcal{G}_{B}\right), T}\right)\right) \\
& =f^{-1}\left(\bigcap_{T>0} \operatorname{cl}\left(f(\mathcal{X}) \cap S_{B \cap f(\mathcal{X}), T}\right) \cup \bigcap_{T>0} \operatorname{cl}\left(f(\mathcal{X}) \cap S_{f\left(\mathcal{G}_{B}\right), T}\right)\right) \\
& =\bigcap_{T>0} f^{-1}\left(\operatorname{cl}\left(f\left(R_{f-1}(B), T\right)\right) \cup \bigcap_{T>0} f^{-1}\left(\operatorname{cl}\left(f\left(R_{\mathcal{G}_{B}, T}\right)\right)\right)\right. \\
& =\bigcap_{T>0} \operatorname{cl}\left(R_{f}-1(B), T\right) \cup \bigcap_{T>0} \operatorname{cl}\left(R_{\mathcal{G}_{B}, T}\right) \\
& =: \omega\left(f^{-1}(B)\right) \cup \omega\left(\mathcal{G}_{B}\right),
\end{aligned}
$$

The first equality follows from taking the preimage of both sides of (11). The second equality follows from the definition of $\omega$-limit set. The third equality follows since intersecting a set with $f(\mathcal{X})$ does not change its $f$-preimage. The fourth equality follows from (12). The fifth equality follows from (15) and the distributivity of preimages over intersections and unions. The sixth equality is justified by the facts that (i) $f$ is a continuous and closed map, so taking $f$-images commutes with taking closures [Lee10, Prop. 2.30], and (ii) $f$ is injective, so $f^{-1}(f(X))=X$ for any $X \subseteq \Sigma_{H}$.

To complete the proof, it remains only to verify (10). Fix $A \subseteq \mathcal{X}$. We have $\mathcal{G}_{f(A)} \subseteq A \cup r^{-1}(A)$ by the definition of $\mathcal{G}_{f(A)}$. Since $\omega\left(r^{-1}(A)\right) \subseteq \omega(A)$ by the definition of $\omega$-limit sets, the finite-union property of $\omega$-limit sets (footnote 12) implies that $\omega\left(\mathcal{G}_{f(A)}\right) \subseteq \omega(A) \cup \omega\left(r^{-1}(A)\right) \subseteq \omega(A)$. Taking $B=f(A)$ in (9), we thus obtain

$$
f^{-1}(\omega(f(A)))=\omega\left(f^{-1}(f(A))\right) \cup \omega\left(\mathcal{G}_{f(A)}\right) .
$$

Since $f$ is injective, $A=f^{-1}(f(A))$; substituting this into the first term on the right above and using $\omega\left(\mathcal{G}_{f(A)}\right) \subseteq \omega(A)$ yields $(10)$. This completes the proof.

Corollary 2. Let $H=(\mathcal{X}, \mathcal{F}, \mathcal{G}, \varphi, r)$ be a THS satisfying Assumptions 1,2 , and 3 with $r: \mathcal{G} \rightarrow \mathcal{X}$ a closed map. Then for any $U \subseteq \mathcal{X}, \omega(U)$ is forward invariant.

\footnotetext{
${ }^{13}$ Proof: $((r(\mathcal{G}) \cap \mathcal{G}) \times\{0\}) \cup\left(r^{-1}(\mathcal{G}) \times\{1\}\right) \subseteq \mathcal{G} \times\{0,1\} \subseteq Y$, so $g^{-1}(g(Y))=Y \cup((r(\mathcal{G}) \cap \mathcal{G}) \times\{0\}) \cup\left(r^{-1}(\mathcal{G}) \times\{1\}\right)=Y$.
} 
Proof. Let all notation be as in Prop. 6 above, let $U \subseteq \mathcal{X}$ be an arbitrary subset, and define $X:=\pi \circ \iota(U)$. Since $\pi \circ \iota$ is injective, it follows that $U=(\pi \circ \iota)^{-1}(X)$. Hence Equation 10 implies that

$$
\omega(U)=(\pi \circ \iota)^{-1}(\omega(X)) .
$$

It is well-known from classical dynamical systems theory that $\omega$-limit sets of continuous semiflows are forward invariant (this is also easy to prove directly), so $\omega(X)$ is forward invariant for $\Phi_{H}$. Since the collection of maximal $H$ executions is precisely the collection of $(\pi \circ \iota)$-preimages of $\Phi_{H}$ trajectories, Equation (16) implies that $\omega(U)$ is forward invariant.

Proposition 7. Let $H=(\mathcal{X}, \mathcal{F}, \mathcal{G}, \varphi, r)$ be a THS satisfying satisfying Assumptions 1,2 , 3, and 4 with $r: \mathcal{G} \rightarrow \mathcal{X}$ a closed map. Let $H^{\prime}=\left(\mathcal{X}^{\prime}, \mathcal{F}^{\prime}, \mathcal{G}^{\prime}, \varphi^{\prime}, r^{\prime}\right)$ be the relaxed hybrid system of Def. 13, $\iota: \mathcal{X} \rightarrow \mathcal{X}^{\prime}$ be the obvious embedding, and let $\left(\Sigma_{H}, \Phi_{H}\right)$ be the hybrid suspension of Def. 14 with quotient $\pi: \mathcal{X}^{\prime} \rightarrow \Sigma_{H}$.

Then $\left(A, A^{*}\right)$ is an attracting-repelling pair for $H$ if and only if $\left(A, A^{*}\right)=\left((\pi \circ \iota)^{-1}(B),(\pi \circ \iota)^{-1}\left(B^{*}\right)\right)$ for some attracting-repelling pair $\left(B, B^{*}\right)$ for $\Phi_{H}$.

Proof. For purposes of readability, for this proof we define $f:=\pi \circ \iota$. We begin by noting that, if $W \subseteq \Sigma_{H}$ is any forward invariant set, then it follows from the definition of $\Phi_{H}$ that

$$
\Phi^{[1+T, \infty)}(W \cap f(\mathcal{X})) \subseteq \Phi^{[1+T, \infty)}(W) \subseteq \Phi^{[T, \infty)}(W \cap f(\mathcal{X}))
$$

for any $T \geq 0$. By the definition of $\omega$-limit set, this implies that

$$
\omega(W)=\omega(W \cap f(\mathcal{X})) .
$$

Now let $U:=f^{-1}(W)$. Since $r$ is a closed map, (17) together with Equation (10) of Prop. 6 yield

$$
f^{-1}(\omega(W))=f^{-1}(\omega(W \cap f(\mathcal{X})))=f^{-1}(\omega(f(U)))=\omega(U) .
$$

Now let $\left(B, B^{*}\right)$ be an attracting-repelling pair for $\Phi_{H}$ and let $W$ be an open trapping neighborhood for $B$. Then $U:=f^{-1}(W)$ is open by continuity of $f$, and $\operatorname{cl}(U) \subseteq f^{-1}(\operatorname{cl}(W))$ by continuity of $f$. Hence clearly $U$ satisfies the conditions of Def. 10 defining a trapping neighborhood, so $U$ determines an attracting set $A:=\omega(U)$. Since $W$ is forward invariant, (18) implies that $A=f^{-1}(\omega(W))=f^{-1}(B)$.

We now show that $A^{*}=f^{-1}\left(B^{*}\right)$. Equation (10) of Prop. 6 implies that $\omega(x)=f^{-1}(\omega(f(x)))$ for all $x \in \mathcal{X}$. Since also $A=f^{-1}(B)$, it follows that, for all $x \in \mathcal{X}$,

$$
[\omega(x) \cap A=\varnothing] \Longleftrightarrow\left[f^{-1}(\omega(f(x))) \cap f^{-1}(B)=\varnothing\right] \Longleftrightarrow[\omega(f(x)) \cap B \cap f(\mathcal{X})=\varnothing] .
$$

The latter in turn holds if and only if $\omega(f(x)) \cap B=\varnothing$, since $\omega(f(x)) \cap B$ is forward invariant and $\Sigma_{H} \backslash f(\mathcal{X})$ contains no nonempty forward invariant subset. Thus, the repelling set $A^{*}$ dual to $A$ is given by $A^{*}=f^{-1}\left(B^{*}\right)$. We have now shown that, for every attracting-repelling pair $\left(B, B^{*}\right)$ for $\Phi_{H}$, $\left(f^{-1}(B), f^{-1}\left(B^{*}\right)\right)$ is an attracting-repelling pair for $H$.

To prove the converse, let $\left(A, A^{*}\right)$ be an attracting-repelling pair for $H$ and let $U \supseteq A$ be an open trapping neighborhood as in Def. 10. Then by the definition of the disjoint union and quotient topologies, $U_{0}^{\prime}:=\pi_{0}(U \sqcup((U \cap \mathcal{G}) \times[0,1]))$ is an open subset of $\mathcal{X}^{\prime}$, where $\pi_{0}: \mathcal{X} \sqcup(\mathcal{G} \times[0,1]) \rightarrow \mathcal{X}^{\prime}$ is the quotient of Def. 13. Now define the set $U^{\prime}:=U_{0}^{\prime} \cup \pi_{0}\left(r^{-1}(U) \times(0,1]\right) \subseteq \mathcal{X}^{\prime}$, which is also open (for similar reasons). $U^{\prime}$ is saturated with respect to $\pi$ since

$$
\pi^{-1}\left(\pi\left(U^{\prime}\right)\right)=r^{\prime}\left(U^{\prime} \cap \mathcal{G}^{\prime}\right) \cup U^{\prime} \cup\left(r^{\prime}\right)^{-1}\left(U^{\prime}\right)=U^{\prime} \cup\left(r^{\prime}\right)^{-1}\left(U^{\prime}\right)=U^{\prime} .
$$

The first equality follows from the definition of $\pi$ and the fact that $r^{\prime}\left(\mathcal{G}^{\prime}\right) \cap \mathcal{G}^{\prime}=\varnothing$. The second equality follows using the definition of $U^{\prime}$ and the fact that $U$ is forward invariant: $r^{\prime}\left(U^{\prime} \cap \mathcal{G}^{\prime}\right)=$ $\iota\left(r(U \cap \mathcal{G}) \cup r\left(r^{-1}(U)\right)\right) \subseteq \iota(U) \subseteq U^{\prime}$, so $r^{\prime}\left(U^{\prime} \cap \mathcal{G}^{\prime}\right) \cup U^{\prime}=U^{\prime}$. The third equality follows since $r^{\prime}\left(\mathcal{G}^{\prime}\right) \subseteq \iota(\mathcal{X})$ and since $U^{\prime} \cap \iota(\mathcal{X})=\iota(U)$, so $\left(r^{\prime}\right)^{-1}\left(U^{\prime}\right)=\left(r^{\prime}\right)^{-1}(\iota(U))=\pi_{0}\left(r^{-1}(U) \times\{1\}\right) \subseteq U^{\prime}$.

Since $U^{\prime}$ is open and saturated, $W:=\pi\left(U^{\prime}\right) \subseteq \Sigma_{H}$ is open, and $\operatorname{cl}(W)=\pi\left(\operatorname{cl}\left(U^{\prime}\right)\right)$ since, by Lemma 2, $\pi$ is a closed map [Lee10, Prop. 2.30]. These facts, together with the definitions of $\Phi_{H}$ and $U^{\prime}$ and the fact that $U^{\prime}$ is a trapping neighborhood, imply that $W$ is a trapping neighborhood for some attracting set $B:=\omega(W)$. Using the definition of $U^{\prime}$ and the fact that $U^{\prime}$ is saturated, it follows that $f^{-1}(W)=U$. Since $W$ is forward invariant, (18) therefore implies that $A=\omega(U)=f^{-1}(\omega(W))=f^{-1}(B)$. Finally, repeating the argument from the first part of the proof verbatim shows that $A^{*}=f^{-1}\left(B^{*}\right)$. Hence every attracting 


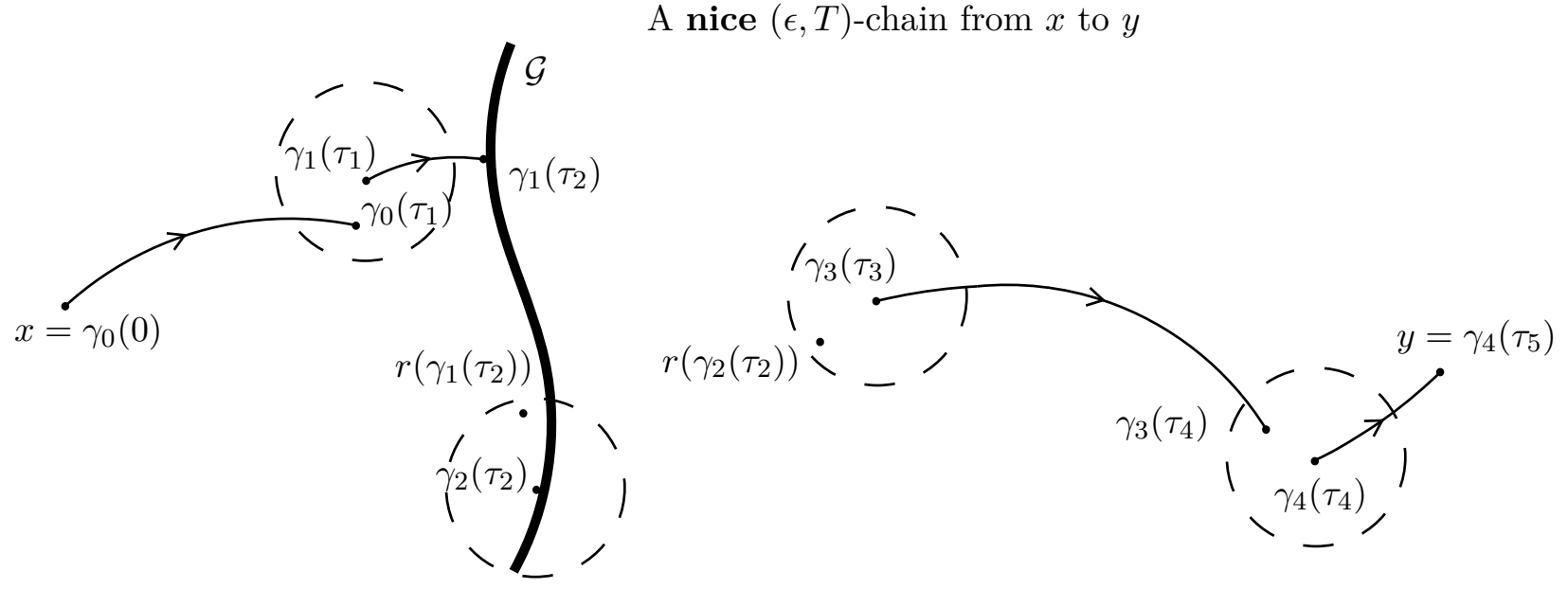

Figure 7. A nice $(\epsilon, T)$-chain from $x \in \mathcal{X}$ to $y \in \mathcal{X}$. Notice that, unlike in the $(\epsilon, T)$-chain of Fig. 2, no "double jumps" are allowed.

repelling-pair $\left(A, A^{*}\right)$ for $H$ is of the form $\left(f^{-1}(B), f^{-1}\left(B^{*}\right)\right)$ for some attracting-repelling pair $\left(B, B^{*}\right)$ for $\Phi_{H}$. This completes the proof.

6.2. Chain equivalence in the hybrid suspension. To prove our main results, we will relate the chain recurrent set of an MHS with the chain recurrent set of its hybrid suspension semiflow. Prop. 8 below establishes this relationship. We prove Prop. 8 using the following three technical lemmas, the proofs of which are deferred to SM $\S \mathrm{D}$.

The first technical lemma (Lemma 4) shows that - under Assumptions 1, 2, 3, and 4-the Conley relation is unaffected by restricting the set of hybrid $(\epsilon, T)$-chains to a "nice" subset for which there must be timeseparation between any reset jump and a subsequent continuous-time jump. In other words, such nice chains are not allowed to have "double jumps" like the one shown in Fig. 2. We make this notion precise in Def. 15; a nice chain is shown in Fig. 7.

Definition 15. Let $H$ be an MHS. We say that a chain $\chi=(N, \tau, \eta, \gamma) \in \mathcal{C} h_{H}^{\epsilon, T}$ is nice if $\tau_{\eta_{k}}-\tau_{\left(\eta_{k}-1\right)} \geq T$ for all $k \geq 1$. We denote the set of nice $(\epsilon, T)$-chains in $H$ by $\widehat{\mathcal{C} h_{H}^{\epsilon, T}}$. We let $\widehat{\mathcal{C} h_{H}}$ denote the Conley relation with respect to the set of nice chains, i.e.,

$$
(x, y) \in \widehat{\mathcal{C} h_{H}} \Longleftrightarrow \text { for all } \epsilon, T>0: \widehat{\mathcal{C} h_{H}^{\epsilon, T}}(x, y) \neq \varnothing .
$$

As in Def. 5 , we sometimes use the more intuitive notation $\widehat{\mathcal{C} h_{H}}(x, y)$ in place of $(x, y) \in \widehat{\mathcal{C} h_{H}}$.

Lemma 4. Let $H=(\mathcal{X}, \mathcal{F}, \mathcal{G}, \varphi, r)$ be a MHS satisfying Assumptions $1,2,3$, and 4. Then

$$
\mathcal{C} h_{H}=\widehat{\mathcal{C} h_{H}} \text {. }
$$

In particular, two points of $\mathcal{X}$ are chain equivalent if and only if they are chain equivalent with respect to nice chains only.

The following lemma shows that-under Assumptions 1, 2, 3, and 4-the Conley relation for $H$ is unaffected by allowing additional jumps to occur within the relaxed hybrid system $H^{\prime}$.

Lemma 5. Let $H=(\mathcal{X}, \mathcal{F}, \mathcal{G}, \varphi, r)$ be an MHS satisfying Assumptions 1, 2, 3, and 4 . Let $H^{\prime}=$ $\left(\mathcal{X}^{\prime}, \mathcal{F}^{\prime}, \mathcal{G}^{\prime}, \varphi^{\prime}, r^{\prime}\right)$ be the associated relaxed hybrid system equipped with any compatible extended metric making $H^{\prime}$ an MHS. Let $\iota: \mathcal{X} \rightarrow \mathcal{X}^{\prime}$ be the obvious embedding. Then for all $x, y \in \mathcal{X}$, we have

$$
\mathcal{C} h_{H}(x, y) \Longleftrightarrow \mathcal{C} h_{H^{\prime}}(\iota(x), \iota(y))
$$

The following lemma is a minor adaptation of [CK00, Prop. B.2.19]. 
Lemma 6. Let $X$ be a compact metric space and $\Phi:[0, \infty) \times X \rightarrow X$ be a continuous semiflow. Consider $x, y \in X$ and fix $T>0$. If for every $\epsilon>0$ there exist $(\epsilon, T)$-chains from (i) $x$ to $y$ and (ii) $y$ to $x$, then $x$ and $y$ are chain equivalent.

Remark 16. Formally speaking, Lemma 6 is discussing $(\epsilon, T)$-chains and chain equivalence as defined in Def. 4 and 7, as opposed to the standard definitions for semiflows (see Ex. 2) to which [CK00, Prop. B.2.19] applies. However, the proofs are similar for either definition. Furthermore, our proof actually directly shows that $x$ and $y$ are also chain equivalent according to the standard definition, but this also follows from the stated conclusion and Ex. 2.

We now come to the main result of this section.

Proposition 8 (The chain equivalence classes of a compact hybrid system and its suspension). Let $H=$ $(\mathcal{X}, \mathcal{F}, \mathcal{G}, \varphi, r)$ be an MHS satisfying Assumptions 1, 2, 3, and 4. Let $H^{\prime}=\left(\mathcal{X}^{\prime}, \mathcal{F}^{\prime}, \mathcal{G}^{\prime}, \varphi^{\prime}, r^{\prime}\right)$ be the relaxed hybrid system of Def. 13, let $\iota: \mathcal{X} \rightarrow \mathcal{X}^{\prime}$ be the obvious embedding, and let $\left(\Sigma_{H}, \Phi_{H}\right)$ be the hybrid suspension of Def. 14 with quotient $\pi: \mathcal{X}^{\prime} \rightarrow \Sigma_{H}$.

Then for any choice of compatible extended metric on $\Sigma_{H}, x, y \in \mathcal{X}$ are chain equivalent for $H$ if and only if $\pi \circ \iota(x), \pi \circ \iota(y) \in \Sigma_{H}$ are chain equivalent for $\Phi_{H}$. In particular, it follows that $R(H)=(\pi \circ \iota)^{-1}\left(R\left(\Phi_{H}\right)\right)$, where $R\left(\Phi_{H}\right)$ is the chain recurrent set for $\Phi_{H}$.

Remark 17. Prop. 8 would become false if the hybrid suspension $\Sigma_{H}$ was replaced by the (generalized) hybrifold $M_{H}$ of $H$ defined in SM §A.4.1. For example, consider a discrete-time dynamical system, i.e., a hybrid system $H$ with $\mathcal{X}=\mathcal{G}$ and $\mathcal{F}=\varnothing$ (cf. Ex. 1). In general $M_{H}$ may not be metrizable (even if $\mathcal{X}$ is a compact metric space), as illustrated by the example of a discrete-time dynamical system given by iterating an irrational rotation of the circle. However, even if $M_{H}$ happens to be metrizable, every point of $M_{H}$ is a stationary point for the (generalized) hybrifold semiflow, and therefore every point of $M_{H}$ is chain recurrent. On the other hand, the chain recurrent set of $H$ is arbitrary.

The following immediate corollary of Prop. 8 concerns the classical suspension of a discrete-time dynamical system and is probably well-known, although we could not find a reference in the literature. See SM $\S \mathrm{B}$ or [Sma67, BS02, p. 797, pp. 21-22] for a primer on the classical suspension semiflow.

Corollary 3. Consider the discrete-time dynamical system defined by a continuous map $f: X \rightarrow X$ of a compact metric space. Let $\Sigma_{f}:=\frac{X \times[0,1]}{(x, 1) \sim(0, f(x))}$ be the classical suspension (mapping torus) of $f$, $\Phi:[0, \infty) \times \Sigma_{f} \rightarrow \Sigma_{f}$ be the suspension semiflow, $\iota: X \rightarrow X \times[0,1]$ be the embedding $X \hookrightarrow X \times\{0\}$, and $\pi: X \times[0,1] \rightarrow \Sigma_{f}$ be the quotient map.

Then for any choice of compatible extended metric on $\Sigma_{f}, x, y \in X$ are chain equivalent for $f$ if and only if $\pi \circ \iota(x), \pi \circ \iota(y)$ are chain equivalent for $\Phi$. In particular, $R(f)=(\pi \circ \iota)^{-1}(R(\Phi))$.

The classical analogue of the following corollary of Prop. 8 is well-known.

Corollary 4. Let $H=(\mathcal{X}, \mathcal{F}, \mathcal{G}, \varphi, r)$ be an MHS satisfying Assumptions 1, 2, 3, and 4. Then the chain equivalence classes of $H$ are closed and forward invariant. Similarly, $R(H)$ is closed and forward invariant. Additionally, $\omega(x) \subseteq R(H)$ for any $x \in \mathcal{X}$.

Proof of Cor. 4. For the standard, classical definition of the Conley relation for the semiflow $\Phi_{H}$ (and for any choice of compatible metric on $\Sigma_{H}$ ), it is well known that all chain equivalence classes for $\Phi_{H}$, as well as the chain recurrent set for $\Phi_{H}$, are closed and forward invariant (this is also easy to prove directly). By Ex. 2, the same is true if our definition of the Conley relation (Def. 5) for $\Phi_{H}$ is used instead. It follows that the same is true for $H$ since (i) Prop. 8 implies that the chain equivalence classes for $H$ (and hence also $R(H)$ ) are ( $\pi \circ \iota$ )-preimages of the chain equivalence classes for $\Phi_{H}$, (ii) $\pi \circ \iota$ is continuous, and (iii) the collection of maximal $H$ executions is precisely the collection of $(\pi \circ \iota)$-preimages of $\Phi_{H}$ trajectories.

Fix $x \in \mathcal{X}$ and define $[x]:=\pi \circ \iota(x)$. The final assertion follows from Prop. 8 and Equation (10) of Prop. 6 (which applies by Remark 14):

$$
\omega(x)=(\pi \circ \iota)^{-1}(\omega([x])) \subseteq(\pi \circ \iota)^{-1}\left(R\left(\Phi_{H}\right)\right)=R(H) .
$$

The set inclusion follows from the well-known fact that, for a semiflow, the chain recurrent set contains the $\omega$-limit set of any point (cf. [Con78, II.6.3.C]). 
Proof of Prop. 8. In the following, we let $d_{\mathcal{X}}$ be the given extended metric on $\mathcal{X}$ and $d_{\mathcal{X}^{\prime}}, d_{\Sigma_{H}}$ be any metrics on $\mathcal{X}^{\prime}, \Sigma_{H}$ which are compatible with their respective topologies, and we use the notation $[x]:=\pi(x)$ for $x \in \mathcal{X}^{\prime}$. Through a mild abuse of notation, we also use the notation $[x]:=\pi \circ \iota(x)$ for $x \in \mathcal{X}$ and $[(z, t)]:=\pi \circ \pi_{0}(z, t)$, where $\pi_{0}: \mathcal{X} \sqcup(\mathcal{G} \times[0,1]) \rightarrow \mathcal{X}^{\prime}$ is the quotient of Def. 13 .

We first show that, if $x, y \in \mathcal{X}$ are chain equivalent, then $[x]$ and $[y]$ are chain equivalent for $\Phi_{H} \cdot{ }^{14}$ Since $\mathcal{X}$ is compact it follows that the map $\pi \circ \iota: \mathcal{X} \rightarrow \Sigma_{H}$ is uniformly continuous with respect to $d_{\mathcal{X}}, d_{\Sigma_{H}}$. This implies that, for any $\epsilon>0$, there exists $\delta>0$ such that every nice $(\delta, T+1)$-chain for $H$ maps to an $(\epsilon, 1)$-chain for $\Phi_{H}$ under $\pi \circ \iota$. Lemma 4 implies that, for every $\delta, T>0$, there are nice $(\delta, T+1)$-chains from (i) $x$ to $y$ and (ii) $y$ to $x$, so it follows that there are $(\epsilon, 1)$-chains from (i) $[x]$ to $[y]$ and (ii) $[y]$ to $[x]$ for every $\epsilon>0$. Hence Lemma 6 implies that $[x]$ and $[y]$ are chain equivalent for $\Phi_{H}$.

To complete the proof we now show that, if $x, y \in R(H)$ are such that $[x]$ and $[y]$ are chain equivalent for $\Phi_{H}$, then $x$ and $y$ are chain equivalent. It suffices to prove the stronger claim that $[x]$ being Conley related to $[y]$ for $\Phi_{H}$ implies $\mathcal{C} h_{H}(x, y)$ for arbitrary $x, y \in \mathcal{X}$. And in order to prove this, by Lemma 5 it suffices to prove that $[x]$ being Conley related to $[y]$ for $\Phi_{H}$ implies $\mathcal{C} h_{H^{\prime}}(\iota(x), \iota(y))$. We prove the latter claim below in two steps which we first briefly describe in the following paragraph. To improve readability in the remainder of the proof, we henceforth use the notation $[S]:=\pi \circ \iota(S) \subseteq \Sigma_{H}$ if $S \subseteq \mathcal{X},[S]:=\pi(S) \subseteq \Sigma_{H}$ if $S \subseteq \mathcal{X}^{\prime}$, and $[S]:=\pi \circ \pi_{0}(S)$ if $S \subseteq \mathcal{X} \sqcup(\mathcal{G} \times[0,1])$

In Step 1 below, we will show that $(\epsilon, T)$-chains for arbitrary $\epsilon, T>0$ can be constructed between $[x],[y] \in \pi \circ \iota(\mathcal{X})$ with the property that no jump points in the chain belong to $\left[\mathcal{G} \times\left(\frac{1}{2}, 1\right)\right]$. We refer to such chains as $(\epsilon, T)$-special chains. In Step 2 we will use Step 1 as a tool to prove that, for any $\epsilon, T>0$, there exists an $(\epsilon, T)$-chain from $x$ to $y$ for the relaxed system $H^{\prime}$ if $[x],[y]$ are Conley related for $\Phi_{H}$. This will show that $\mathcal{C} h_{H^{\prime}}(x, y)$, and hence $\mathcal{C} h_{H}(x, y)$ by Lemma 5 , as desired.

Step 1: Define $U^{\prime}:=\pi_{0}\left(\mathcal{G} \times\left[\frac{1}{4}, 1\right]\right) \subseteq \mathcal{X}^{\prime}, V^{\prime}:=\pi_{0}\left(\mathcal{G} \times\left(\frac{1}{2}, 1\right)\right) \subseteq U^{\prime}$, and the continuous maps $\rho^{\prime}: U^{\prime} \rightarrow \mathcal{G}^{\prime}$ via $\rho^{\prime}\left(\pi_{0}(z, t)\right):=\pi_{0}(z, 1)$ and $\mu^{\prime}: U^{\prime} \rightarrow\left[0, \frac{3}{4}\right]$ via $\mu^{\prime}\left(\pi_{0}(z, t)\right):=1-t$. Since $U^{\prime}$ and $\left[U^{\prime}\right]$ are compact metrizable spaces, $\left.\pi\right|_{U^{\prime}}: U^{\prime} \rightarrow\left[U^{\prime}\right]$ is a quotient map since it is a continuous, closed, and surjective map. The maps $\left.\pi\right|_{U^{\prime}} \circ \rho^{\prime}$ and $\mu^{\prime}$ are both constant on fibers of $\left.\pi\right|_{U^{\prime}}$ since the restriction of $\pi$ to $\pi_{0}\left(\mathcal{G} \times\left[\frac{1}{4}, 1\right)\right)$ is injective, $\left.\mu^{\prime}\right|_{\mathcal{G}^{\prime}} \equiv 0$, and $\left.\left(\left.\pi\right|_{U^{\prime}} \circ \rho^{\prime}\right)\right|_{\mathcal{G}^{\prime}}=\left.\pi\right|_{\mathcal{G}^{\prime}}$ since $\left.\rho^{\prime}\right|_{\mathcal{G}^{\prime}}=\mathrm{id}_{\mathcal{G}^{\prime}}$. Thus, by the universal property of the quotient topology [Lee10, Thm 3.70], $\left.\pi\right|_{U^{\prime}} \circ \rho^{\prime}$ and $\mu^{\prime}$ descend to a continuous retraction $\nu:\left[U^{\prime}\right] \rightarrow\left[\mathcal{G}^{\prime}\right]$ and a continuous map $\alpha:\left[U^{\prime}\right] \rightarrow\left[0, \frac{3}{4}\right]$, respectively. Note that $\nu$ preserves trajectories, and that $\alpha$ is the "time-to-impact- $\left[\mathcal{G}^{\prime}\right]$ map" for points in $\left[U^{\prime}\right]$.

Now fix $\epsilon, T>0$ and let $[x],[y] \in[\mathcal{X}]$ be Conley related for $\Phi_{H}$. Since $\left[U^{\prime}\right], \Sigma_{H}$, and $\operatorname{cl}\left(\left[V^{\prime}\right]\right)$ are compact, there exists $\delta \in(0, \epsilon)$ such that

$$
\begin{aligned}
{[x],[y] \in\left[U^{\prime}\right], d_{\Sigma_{H}}([x],[y])<\delta } & \Longrightarrow d_{\Sigma_{H}}(\nu([x]), \nu([y]))<\epsilon \\
{[x] \in\left[V^{\prime}\right],[y] \in \Sigma_{H} \backslash \operatorname{int}\left(\left[U^{\prime}\right]\right), d_{\Sigma_{H}}([x],[y])<\delta } & \Longrightarrow d_{\Sigma_{H}}(\nu([x]),[y])<\epsilon .
\end{aligned}
$$

Indeed, suppose there did not exist $\delta>0$ such that (20) held. Then there exist sequences $\left(v_{n}\right)_{n \in \mathbb{N}} \subseteq\left[V^{\prime}\right]$ and $\left(w_{n}\right)_{n \in \mathbb{N}} \subseteq \Sigma_{H} \backslash \operatorname{int}\left(\left[U^{\prime}\right]\right)$ with $d_{\Sigma_{H}}\left(v_{n}, w_{n}\right) \rightarrow 0$ and $d_{\Sigma_{H}}\left(\nu\left(v_{n}\right), w_{n}\right) \geq \epsilon$ for all $n$. By passing to subsequences, we may assume $v_{n} \rightarrow v \in \operatorname{cl}\left(\left[V^{\prime}\right]\right)$ and $w_{n} \rightarrow w \in \Sigma_{H} \backslash \operatorname{int}\left(\left[U^{\prime}\right]\right)$ with $d_{\Sigma_{H}}(\nu(v), w) \geq \epsilon$. Since $d_{\Sigma_{H}}\left(v_{n}, w_{n}\right) \rightarrow 0$, we have $v=w$. Thus, $v \in \operatorname{cl}\left(\left[V^{\prime}\right]\right) \cap\left(\Sigma_{H} \backslash \operatorname{int}\left(\left[U^{\prime}\right]\right)\right)=\left[\mathcal{G}^{\prime}\right]$ and $d_{\Sigma_{H}}(\nu(v), v) \geq \epsilon$, a contradiction since $\left.\nu\right|_{\left[\mathcal{G}^{\prime}\right]}=\mathrm{id}_{\left[\mathcal{G}^{\prime}\right]}$.

Let $^{15} \chi^{(0)}=\left(N, \tau^{(0)}, \eta^{(0)}, \gamma^{(0)}\right) \in \mathcal{C} h_{\Phi_{H}}^{\delta, T+3 / 4}([x],[y])$; recall from Def. 4 that $N \geq 1$. We will now modify the chain $\chi^{(0)}$ inductively. Fix $i \in\{0, \ldots, N-1\}$ and assume that, if $i \geq 1,{ }^{16}$ we have modified the first $(i+1) \operatorname{arcs}\left(\gamma_{0}^{(0)}, \ldots, \gamma_{i}^{(0)}\right)$ to obtain a chain $\chi^{(i)}=\left(N, \tau^{(i)}, \eta^{(i)}, \gamma^{(i)}\right) \in \mathcal{C} h_{\Phi_{H}}^{\epsilon, T}([x],[y])$ such that (a) the

\footnotetext{
${ }^{14}$ In this proof we are using Def. 4 and 5 for the definition of the Conley relation for $\Phi_{H}$, although Ex. 2 shows that this is equivalent to the classical definition.

${ }^{15}$ The semiflow $\Phi_{H}$ defines an MHS $\widetilde{H}$ with guard $\varnothing$ (cf. Ex. 2). To avoid introducing extra notation, here we conflate $\Phi_{H}$ with $\widetilde{H}$ by writing, e.g., $\mathcal{C} h_{\Phi_{H}}^{\delta, T+3 / 4}([x],[y])$ instead of $\mathcal{C} h_{\widetilde{H}}^{\delta, T+3 / 4}([x],[y])$. We additionally remark that, since the guard for $\widetilde{H}$ is $\varnothing$, chains can only have continuous-time jumps, so every chain $\chi=(N, \tau, \eta, \gamma)$ for $\Phi_{H}$ satisfies $\eta=(0,1,2, \ldots, N)$.

${ }^{16}$ If $i=0$ we assume nothing, so that the base case of the induction argument is included in this one.
} 
sub-chain obtained by throwing away the first $(i+1) \operatorname{arcs}$ of $\chi^{(i)}$ is either a single arc (if $i=N-1$ ) or a $(\delta, T+3 / 4)$-chain; and (b) for all $j \in\{1, \ldots, i\}: \gamma_{j-1}^{(i)}\left(\tau_{j}^{(i)}\right), \gamma_{j}^{(i)}\left(\tau_{j}^{(i)}\right) \notin\left[V^{\prime}\right]$.

- If both $\gamma_{i}^{(i)}\left(\tau_{i+1}^{(i)}\right), \gamma_{i+1}^{(i)}\left(\tau_{i+1}^{(i)}\right) \in\left[U^{\prime}\right]$, then we replace $\gamma_{i}^{(i)}$ with the arc

$$
\left[\tau_{i}^{(i)}, \tau_{i+1}^{(i)}+\alpha\left(\gamma_{i}^{(i)}\left(\tau_{i+1}^{(i)}\right)\right)\right] \ni t \mapsto \Phi_{H}^{t-\tau_{i}^{(i)}}\left(\gamma_{i}^{(i)}\left(\tau_{i}^{(i)}\right)\right)
$$

and $\gamma_{i+1}^{(i)}$ with the arc

$$
\left[\tau_{i+1}^{(i)}+\alpha\left(\gamma_{i}^{(i)}\left(\tau_{i+1}^{(i)}\right)\right), \tau_{i+2}^{(i)}\right] \ni t \mapsto \Phi_{H}^{t+\alpha\left(\gamma_{i+1}^{(i)}\left(\tau_{i+1}^{(i)}\right)\right)-\tau_{i+1}^{(i)}-\alpha\left(\gamma_{i}^{(i)}\left(\tau_{i+1}^{(i)}\right)\right)}\left(\gamma_{i+1}^{(i)}\left(\tau_{i+1}^{(i)}\right)\right) .
$$

- If $\gamma_{i}^{(i)}\left(\tau_{i+1}^{(i)}\right) \in\left[V^{\prime}\right]$ and $\gamma_{i+1}^{(i)}\left(\tau_{i+1}^{(i)}\right) \in \Sigma_{H} \backslash \operatorname{int}\left(\left[U^{\prime}\right]\right)$, then we replace $\gamma_{i}^{(i)}$ with the arc defined by (21), but we do not modify $\gamma_{i+1}^{(i)}$.

- If $\gamma_{i+1}^{(i)}\left(\tau_{i+1}^{(i)}\right) \in\left[V^{\prime}\right]$ and $\gamma_{i}^{(i)}\left(\tau_{i+1}^{(i)}\right) \in \Sigma_{H} \backslash \operatorname{int}\left(\left[U^{\prime}\right]\right)$, then we replace $\gamma_{i+1}^{(i)}$ with the arc defined by (22), but we do not modify $\gamma_{i}^{(i)}$.

The upper bound $\alpha(\cdot) \leq \frac{3}{4}$ and Equations (19), (20) can be used to show that, after redefining the sequences $\eta^{(i)}$ and $\tau^{(i)}$ accordingly, the result is a chain $\chi^{(i+1)}=\left(N, \tau^{(i+1)}, \eta^{(i+1)}, \gamma^{(i+1)}\right) \in \mathcal{C} h_{\Phi_{H}}^{\epsilon, T}([x],[y])$ such that (a) the sub-chain obtained by throwing away the first $(i+2)$ arcs of $\chi^{(i+1)}$ is either empty (if $i=N-1$ ), a single arc (if $i=N-2$ ), or a $(\delta, T+3 / 4)$-chain; and (b) for all $j \in\{1, \ldots, i+1\}$ : $\gamma_{j-1}^{(i+1)}\left(\tau_{j}^{(i+1)}\right), \gamma_{j}^{(i+1)}\left(\tau_{j}^{(i+1)}\right) \notin\left[V^{\prime}\right]$.

Hence by induction we obtain a chain $\chi \in \mathcal{C} h_{\Phi_{H}}^{\epsilon, T}([x],[y])$ satisfying $\gamma_{i}\left(\tau_{i}\right), \gamma_{i}\left(\tau_{i+1}\right) \notin\left[V^{\prime}\right]$ for every arc of $\chi$. This shows that there exists an $(\epsilon, T)$-special chain from $[x]$ to $[y]$ and completes the proof of Step 1.

Step 2: Fix $\epsilon>0$ and define $W^{\prime}:=\iota(\mathcal{X}) \cup \pi_{0}\left(\mathcal{G} \times\left[0, \frac{1}{2}\right]\right) \subseteq \mathcal{X}^{\prime}$. Since $W^{\prime}$ is compact and $\left.\pi\right|_{W^{\prime}}$ is injective, $\left.\pi\right|_{W^{\prime}}: W^{\prime} \rightarrow\left[W^{\prime}\right]=\Sigma_{H} \backslash\left[V^{\prime}\right]$ is a homeomorphism of compact metric spaces [Lee10, Lem 4.50.d]. It follows that the inverse homeomorphism $\left(\left.\pi\right|_{W^{\prime}}\right)^{-1}: \Sigma_{H} \backslash\left[V^{\prime}\right] \rightarrow W^{\prime}$ is uniformly continuous, so there exists $\delta>0$ such that $d_{\Sigma_{H}}([z],[w])<\delta$ implies that $d_{\mathcal{X}^{\prime}}(z, w)<\epsilon$ for all $z, w \in W^{\prime}$.

Now fix $\epsilon, T>0$, let $\delta>0$ as in the above paragraph, and let $x, y \in \mathcal{X}$ be such that $[x],[y] \in[\mathcal{X}] \subseteq\left[W^{\prime}\right]$ are Conley related for $\Phi_{H}$. By Step 1, there exists a $(\delta, T)$-special chain $\chi=(N, \tau, \eta, \gamma)$ from $[x]$ to $[y]$. Since $\left.\pi\right|_{W^{\prime}}$ is a homeomorphism onto its image, we can define a sequence of "lifted and reset-subdivided" continuous $\operatorname{arcs}\left(\tilde{\gamma}_{j}\right)$ in $\mathcal{X}^{\prime}$ by first lifting each component of $\gamma_{i}^{-1}\left(\left[W^{\prime}\right]\right)$ via $\left(\left.\pi\right|_{W^{\prime}}\right)^{-1}$, then extending each lifted component terminating at a point $\pi_{0}\left(z, \frac{1}{2}\right)$ in the boundary of $W^{\prime}$ via concatenation with $\left(t \in\left[0, \frac{1}{2}\right] \mapsto \pi_{0}\left(z, t+\frac{1}{2}\right)\right)$. Since $d_{\Sigma_{H}}\left(\gamma_{i}\left(\tau_{i+1}\right), \gamma_{i+1}\left(\tau_{i+1}\right)\right)<\delta$ for each $i$, it follows from our choice of $\delta$ that the resulting family $\left(\tilde{\gamma}_{j}\right)$ of arcs yields an $(\epsilon, T)$-chain for $H^{\prime} .{ }^{17}$ This shows that $x, y \in \mathcal{X}$ are Conley related for $H^{\prime}$. By Lemma 5, this shows that $x, y \in \mathcal{X}$ are also Conley related for $H$ and completes the proof.

6.3. Proofs of Theorems 1 and 2. We are now in a position to prove our main theorems, which we restate for convenience.

Theorem 1 (Conley's decomposition theorem for MHS). Let $H=(\mathcal{X}, \mathcal{F}, \mathcal{G}, \varphi, r)$ be a metric hybrid system satisfying Assumptions 1, 2, 3, and 4. Then the hybrid chain recurrent set $R(H)$ admits a Conley decomposition:

$$
R(H)=\bigcap\left\{A \cup A^{*} \mid A \text { is an attracting set for } H .\right\} .
$$

Furthermore, $x, y \in \mathcal{X}$ are chain equivalent if and only if either $x, y \in A$ or $x, y \in A^{*}$ for every attractingrepelling pair $\left(A, A^{*}\right)$.

\footnotetext{
${ }^{17}$ For this step of the proof it is crucial that our definition of $(\epsilon, T)$-chains (Def. 4) allows for "double jumps," as illustrated in Fig. 2.
} 
Proof. Let $H^{\prime}=\left(\mathcal{X}^{\prime}, \mathcal{F}^{\prime}, \mathcal{G}^{\prime}, \varphi^{\prime}, r^{\prime}\right)$ and $\left(\Sigma_{H}, \Phi_{H}\right)$ be the relaxed hybrid system and hybrid suspension of Def. 13 and 14 (equipped with any compatible extended metrics), $\iota: \mathcal{X} \rightarrow \mathcal{X}^{\prime}$ be the obvious embedding, and $\pi: \mathcal{X}^{\prime} \rightarrow \Sigma_{H}$ be the quotient map of Def. 14. Letting $R\left(\Phi_{H}\right)$ denote the chain recurrent set for $\Phi_{H}$, the Conley decomposition theorem for semiflows [Hur95, Thm 2] and Ex. 2 imply that

$$
R\left(\Phi_{H}\right)=\bigcap\left\{B \cup B^{*} \mid B \text { is an attracting set for } \Phi_{H} \cdot\right\},
$$

and that $\pi \circ \iota(x)$ is chain equivalent to $\pi \circ \iota(y)$ if and only if either $\pi \circ \iota(x), \pi \circ \iota(y) \in B$ or $\pi \circ \iota(x), \pi \circ \iota(y) \in B^{*}$ for every attracting-repelling pair $\left(B, B^{*}\right)$ for $\Phi_{H}$. Prop. 8 implies that $R(H)=(\pi \circ \iota)^{-1}\left(R\left(\Phi_{H}\right)\right)$ and, furthermore, that the chain equivalence classes of $H$ are precisely the $(\pi \circ \iota)$-preimages of chain equivalence classes for $\Phi_{H}$. Hence to complete the proof it would suffice to show that $\left(A, A^{*}\right)$ is an attracting-repelling pair for $H$ if and only if $\left(A, A^{*}\right)=\left((\pi \circ \iota)^{-1}(B),(\pi \circ \iota)^{-1}\left(B^{*}\right)\right)$ for some attracting-repelling pair $\left(B, B^{*}\right)$ for $\Phi_{H}$, but this is the content of Prop. 7. This completes the proof.

Theorem 2 (Conley's fundamental theorem for MHS). Let $H=(\mathcal{X}, \mathcal{F}, \mathcal{G}, \varphi, r)$ be a metric hybrid system satisfying Assumptions 1, 2, 3, and 4. Then there exists a complete Lyapunov function for $H$.

Proof. Let $H^{\prime}=\left(\mathcal{X}^{\prime}, \mathcal{F}^{\prime}, \mathcal{G}^{\prime}, \varphi^{\prime}, r^{\prime}\right)$ be the relaxed hybrid system of Def. 13 and $\iota: \mathcal{X} \rightarrow \mathcal{X}^{\prime}$ the obvious embedding, and let $\left(\Sigma_{H}, \Phi_{H}\right)$ be the hybrid suspension of Def. 14 with quotient $\pi: \mathcal{X}^{\prime} \rightarrow \Sigma_{H}$.

By Prop. $5, \Sigma_{H}$ is compact and metrizable. Since $\Sigma_{H}$ is compact, the Conley relation is independent of the choice of compatible extended metric on $\Sigma_{H}$ (see Remark 5). Hence (after equipping $\Sigma_{H}$ with any compatible extended metric and appealing to Ex. 2) we may apply the fundamental theorem of dynamical systems for semiflows [Pat11, Thm 1.1] to conclude that there exists a complete Lyapunov function $V: \Sigma_{H} \rightarrow \mathbb{R}$ for $\Phi_{H}$. Letting $R\left(\Phi_{H}\right)$ denote the chain recurrent set for $\Phi_{H}$, this means that $V$ is a continuous function such that (i) $t \mapsto V\left(\Phi_{H}^{t}(\pi(x))\right.$ ) is strictly decreasing for all $\pi(x) \notin R\left(\Phi_{H}\right)$, (ii) $V\left(R\left(\Phi_{H}\right)\right.$ ) is nowhere dense in $\mathbb{R}$, and (iii) for all $\pi(x), \pi(y) \in R\left(\Phi_{H}\right): \pi(x)$ and $\pi(y)$ are chain equivalent if and only if $V(\pi(x))=V(\pi(y))$.

Define $L: \mathcal{X} \rightarrow \mathbb{R}$ via $L:=V \circ \pi \circ \iota ; L$ is continuous since $L$ is a composition of continuous functions. By construction, we have that (i) for every $x \in \mathcal{F} \backslash(\pi \circ \iota)^{-1}\left(R\left(\Phi_{H}\right)\right), t>0$, and $y \in \chi_{x}(t), L(y)<L(x)$; and (ii) if $x \in \mathcal{G} \backslash(\pi \circ \iota)^{-1}\left(R\left(\Phi_{H}\right)\right)$, then $L(r(x))<L(x)$. Prop. 8 implies that $\pi \circ \iota(R(H)) \subseteq R\left(\Phi_{H}\right)$, so $L(R(H))=V \circ \pi \circ \iota(R(H)) \subseteq V\left(R\left(\Phi_{H}\right)\right)$; therefore, $L(R(H))$ is nowhere dense in $\mathbb{R}$. It remains only to show that $x, y \in R(H)$ are chain equivalent if and only if $L(x)=L(y)$. Prop. 8 implies that $x, y \in R(H)$ if and only if $\pi \circ \iota(x), \pi \circ \iota(y) \in R\left(\Phi_{H}\right)$, and that $x, y \in R(H)$ are chain equivalent if and only if $\pi \circ \iota(x), \pi \circ \iota(y) \in R\left(\Phi_{H}\right)$ are chain equivalent. By the previous paragraph, $\pi \circ \iota(x), \pi \circ \iota(y) \in R\left(\Phi_{H}\right)$ are chain equivalent if and only if $V(\pi \circ \iota(x))=V(\pi \circ \iota(y))$. Since $L=V \circ \pi \circ \iota$, we have shown that $x, y \in R(H)$ are chain equivalent if and only if $L(x)=L(y)$.

\section{Conclusion}

Using the language of hybrid systems, we have obtained a simultaneous generalization (Theorem 2) of both the continuous-time and discrete-time versions of Conley's fundamental theorem [Con78, Fra88]. As in the classical setting, our theorem asserts the existence of a globally-defined complete Lyapunov function (Def. 12). We have also proved a result (Theorem 1) generalizing Conley's decomposition theorem, which asserts that the chain recurrent set (Def. 6) is the intersection of all attracting-repelling pairs (Def. 10).

While this unification of the continuous and discrete is pleasingly parsimonious, our motivation is not merely parsimony for its own sake. Our long-term aim, motivated particularly by applications in robotics and biomechanics - bearing not simply on legged locomotion [HFKG06, RK15, SKR ${ }^{+} 17$ ], but, indeed, central to the larger prospects for a physically grounded, sensorimotor-coherent, and theoretically sound disciplinary foundation [Kod21, Sec. 4.1.2] - is to continue advancing the program ${ }^{18}$ of developing hybrid dynamical systems theory to the same footing as its more mathematically mature parents, the theories of continous-time and discrete-time dynamical systems. For example, beyond the constructive applications of Conley's theorems discussed in the introduction, practitioners might well choose to use their appearance

\footnotetext{
${ }^{18}$ This program has contributions from many investigators. We only mention a few: [BGM93, Guc95, YMH98, AHLP00, SJSL00, SJLS01, SSJL02, LJS ${ }^{+}$03, SJLS05, HTP05, BRS15, GST09, Ler16, JBK16, BSKR16, BLC18, BC18, CBC19, CB20, LS20].
} 
as a kind of litmus test against which to judge the relative merits of the many different hybrid systems models that have appeared in the literature. Models that do not possess such a decomposition into chain-recurrent and gradient-like parts might be subject to greater scrutiny - their questionably disorderly behavior only tolerated in consequence of expressing some physical property essential to the phenomena of interest. ${ }^{19}$ As a case in point, the absence of any viable notion of steady state behavior occasioned by the departure from the trapping guard condition (Def. 11) of Ex. 3 (see Fig. 3) begins to suggest the potentially wild incoherence of otherwise seemingly well-formulated THS. Thus, by the same token, we hope that our presentation of sufficient conditions for a Conley theory of hybrid systems may encourage more theorists to help determine which properties are necessary. Indeed, despite the ubiquity of hybrid systems in engineering, mathematicians have mostly avoided them, perhaps due to the lack of a single concise definition. In this light, one contribution of the current paper is a parsimonious definition partially generalizing a physically important [JBK16] class of hybrid systems (Def. 1), which we hope may be more inviting to the mathematically inclined reader.

Norton [Nor95] emphasized that the Fundamental Theorem of Dynamical Systems is not the end of the theory but the beginning. Just as the Fundamental Theorems of Arithmetic, Algebra, and Calculus provide the most basic tools of their respective fields, the Fundamental Theorem of Dynamical Systems indicates that the coarsest building blocks of dynamical systems are the countable components of the steady state (the "chain-recurrent") set and their basins (adding in the components of the "gradient-like" sets that lead to them). The primary results of this paper show that these same building blocks fit together in the same way to describe a broad, physically important class of hybrid dynamical systems.

Acknowledgments. This work is supported in part by the Army Research Office (ARO) under the SLICE Multidisciplinary University Research Initiatives (MURI) Program, award W911NF1810327; in part by UATL 10601110D8Z, a LUCI Fellowship granted by the Basic Research Office of the US Undersecretary of Defense for Research and Engineering; and in part by ONR grant N00014-16-1-2817, a Vannevar Bush Fellowship held by the last author, sponsored by the Basic Research Office of the Assistant Secretary of Defense for Research and Engineering. The authors also gratefully acknowledge helpful conversations with Y. Baryshnikov, S. A. Burden, Z. Cooperband, J. Culbertson, D. Guralnik, A. M. Johnson, E. Lerman, and P. F. Stiller. We owe special gratitude to Culbertson for his careful reading of the manuscript; his generosity has spared the reader several ambiguities and typographical errors. We thank the two anonymous referees for useful suggestions.

\section{REFERENCES}

[AC84] J-P Aubin and A Cellina, Differential inclusions: set-valued maps and viability theory, vol. 264, Springer-Verlag, 1984.

$\left[\mathrm{ACR}^{+}\right.$02] E U Acar, H Choset, A A Rizzi, P N Atkar, and D Hull, Morse decompositions for coverage tasks, The International Journal of Robotics Research 21 (2002), no. 4, 331-344.

[AH15] S Awodey and R Harper, Homotopy type theory: Unified foundations of mathematics and computation, ACM SIGLOG News 2 (2015), no. 1, 37-44.

[AHLP00] R Alur, T A Henzinger, G Lafferriere, and G J Pappas, Discrete abstractions of hybrid systems, Proceedings of the IEEE 88 (2000), no. 7, 971-984.

$\left[\mathrm{AKK}^{+}\right.$09] Z Arai, W Kalies, H Kokubu, K Mischaikow, H Oka, and P Pilarczyk, A database schema for the analysis of global dynamics of multiparameter systems, SIAM Journal on Applied Dynamical Systems 8 (2009), no. 3, $757-789$.

[AN07] J M Alongi and G S Nelson, Recurrence and topology, vol. 85, American Mathematical Soc., 2007.

[AS05] A D Ames and S Sastry, A homology theory for hybrid systems: Hybrid homology, International Workshop on Hybrid Systems: Computation and Control, Springer, 2005, pp. 86-102.

[AZGS06] A D Ames, H Zheng, R D Gregg, and S Sastry, Is there life after Zeno? taking executions past the breaking (Zeno) point, 2006 American control conference, IEEE, 2006, pp. 6-pp.

[BC18] S A Burden and S D Coogan, Generalizing infinitesimal contraction analysis to hybrid systems, arXiv preprint arXiv:1804.04122 (2018).

[BCC17] A Bloch, W Clark, and L Colombo, Quasivelocities and symmetries in simple hybrid systems, 2017 IEEE 56th Annual Conference on Decision and Control (CDC), IEEE, 2017, pp. 1529-1534.

[BDJK15] A L Brill, A De, A M Johnson, and D E Koditschek, Tail-assisted rigid and compliant legged leaping, Intelligent Robots and Systems (IROS), 2015 IEEE/RSJ International Conference on, IEEE, 2015, pp. 6304-6311.

\footnotetext{
${ }^{19}$ See the second paragraph of SM $\S A .1$ for a relevant discussion.
} 
[BGM93] A Back, J Guckenheimer, and M Myers, A dynamical simulation facility for hybrid systems, Hybrid Systems (1993), 255-267.

$\left[\mathrm{BGV}^{+} 15\right] \quad \mathrm{S}$ A Burden, H Gonzalez, R Vasudevan, R Bajcsy, and S S Sastry, Metrization and simulation of controlled hybrid systems, IEEE Transactions on Automatic Control 60 (2015), no. 9, 2307-2320.

[BK88] I U Bronštein and A Ya Kopanskii, Chain recurrence in dynamical systems without uniqueness, Nonlinear Analysis: Theory, Methods \& Applications 12 (1988), no. 2, 147-154.

[BK06] H Ban and W D Kalies, A computational approach to Conley's decomposition theorem, Journal of Computational and Nonlinear Dynamics 1 (2006), 312.

[BLC18] S A Burden, T Libby, and S D Coogan, On contraction analysis for hybrid systems, arXiv preprint arXiv:1811.03956 (2018).

[BPS01] M E Broucke, C C Pugh, and S N Simić, Structural stability of piecewise smooth systems, Computational and applied mathematics 20 (2001), no. 1-2, 51-89.

[BRK95] R R Burridge, A A Rizzi, and D E Koditschek, Toward a systems theory for the composition of dynamically dexterous behaviors, 7th Internatinal Symposium on Robotics Research (1995), 534-555.

[BRS15] S A Burden, S Revzen, and S S Sastry, Model reduction near periodic orbits of hybrid dynamical systems, IEEE Transactions on Automatic Control 60 (2015), no. 10, 2626-2639.

[BS02] M Brin and G Stuck, Introduction to dynamical systems, Cambridge University press, 2002.

[BSKR16] S A Burden, S S Sastry, D E Koditschek, and S Revzen, Event-selected vector field discontinuities yield piecewisedifferentiable flows, SIAM Journal on Applied Dynamical Systems 15 (2016), no. 2, 1227-1267.

[CB20] W Clark and A M Bloch, A Poincaré-Bendixson theorem for hybrid dynamical systems on directed graphs, Mathematics of Control, Signals, and Systems 32 (2020), no. 1, 1.

[CBC19] W Clark, A Bloch, and L Colombo, A Poincaré-Bendixson theorem for hybrid systems, Mathematical Control \& Related Fields 10 (2019), no. 1, 27-45.

[CGKS19] J Culbertson, P Gustafson, D E Koditschek, and P F Stiller, Formal composition of hybrid systems, arXiv preprint arXiv:1911.01267 (2019).

[CK00] F Colonius and W Kliemann, The dynamics of control, Springer Science \& Business Media, 2000.

$\left[\mathrm{CML}^{+} 07\right]$ G Chen, K Mischaikow, R S Laramee, P Pilarczyk, and E Zhang, Vector field editing and periodic orbit extraction using Morse decomposition, IEEE Transactions on Visualization and Computer Graphics 13 (2007), no. 4, $769-785$

[Con78] C C Conley, Isolated invariant sets and the Morse index, no. 38, American Mathematical Society, 1978.

[Cow07] N J Cowan, Navigation functions on cross product spaces, IEEE Transactions on Automatic Control 52 (2007), no. $7,1297$.

[DBK18] A De, S A Burden, and D E Koditschek, A hybrid dynamical extension of averaging and its application to the analysis of legged gait stability: The International Journal of Robotics Research 37 (2018), no. 2-3, 266-286.

[FA18] P Forni and D Angeli, Perturbation theory and singular perturbations for input-to-state multistable systems on manifolds, IEEE Transactions on Automatic Control 64 (2018), no. 9, 3555-3570.

[FM88] R D Franzosa and K Mischaikow, The connection matrix theory for semiflows on (not necessarily locally compact) metric spaces, Journal of Differential Equations 71 (1988), no. 2, 270-287.

[Fra88] J Franks, A variation on the Poincaré-Birkhoff theorem, Contemporary Mathematics 81 (1988), 111-116.

[GAP01] J W Grizzle, G Abba, and F Plestan, Asymptotically stable walking for biped robots: Analysis via systems with impulse effects, IEEE Transactions on automatic control 46 (2001), no. 1, 51-64.

[GH15] P Giesl and S Hafstein, Computation and verification of Lyapunov functions, SIAM Journal on Applied Dynamical Systems 14 (2015), no. 4, 1663-1698.

[GS16] R Goebel and R G Sanfelice, How well-posedness of hybrid systems can extend beyond Zeno times, 2016 IEEE 55th Conference on Decision and Control (CDC), IEEE, 2016, pp. 598-603.

[GST09] R Goebel, R G Sanfelice, and A Teel, Hybrid dynamical systems, Control Systems, IEEE 29 (2009), no. 2, 28-93.

[Guc95] J Guckenheimer, A robust hybrid stabilization strategy for equilibria, IEEE Transactions on Automatic Control 40 (1995), no. 2, 321-326.

[GVdBV03] R W Ghrist, J B Van den Berg, and R C Vandervorst, Morse theory on spaces of braids and Lagrangian dynamics, Inventiones mathematicae 152 (2003), no. 2, 369-432.

[Hat01] A Hatcher, Algebraic topology, 1 ed., Cambridge University Press, 2001.

[HCK11] G C Haynes, F R Cohen, and D E Koditschek, Gait transitions for quasi-static hexapedal locomotion on level ground, p. 105-121, Springer, 2011.

[HFKG06] P Holmes, R J Full, D E Koditschek, and J Guckenheimer, The dynamics of legged locomotion: Models, analyses, and challenges, SIAM Review 48 (2006), no. 2, 207-304.

[Hol90] P Holmes, Poincaré, celestial mechanics, dynamical-systems theory and "chaos", Physics Reports 193 (1990), no. 3, 137-163.

[HRK12] G C Haynes, A A Rizzi, and D E Koditschek, Multistable phase regulation for robust steady and transitional legged gaits, The International Journal of Robotics Research 31 (2012), no. 14, 1712- 1738.

[HS06] M W Hirsch and H Smith, Monotone dynamical systems, Handbook of differential equations: ordinary differential equations, vol. 2, Elsevier, 2006, pp. 239-357. 
[HTP05] E Haghverdi, P Tabuada, and G J Pappas, Bisimulation relations for dynamical, control, and hybrid systems, Theoretical Computer Science 342 (2005), no. 2-3, 229-261.

[Hur95] M Hurley, Chain recurrence, semiflows, and gradients, Journal of Dynamics and Differential Equations 7 (1995), no. $3,437-456$

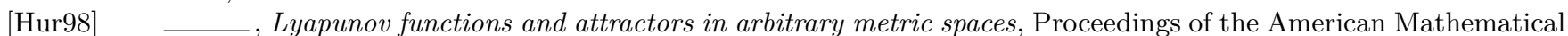
Society 126 (1998), no. 1, 245-256.

[JBK16] A M Johnson, S A Burden, and D E Koditschek, A hybrid systems model for simple manipulation and selfmanipulation systems, The International Journal of Robotics Research 35 (2016), no. 11, 1354-1392.

[JELS99] Karl H Johansson, M Egerstedt, J Lygeros, and S Sastry, On the regularization of Zeno hybrid automata, Systems \& control letters 38 (1999), no. 3, 141-150.

[JK13] A M Johnson and D E Koditschek, Toward a vocabulary of legged leaping, Proceedings of the 2013 IEEE Intl. Conference on Robotics and Automation, May 2013, pp. 2553-2560.

[Kel15] C M Kellett, Classical converse theorems in Lyapunov's second method, Discrete \& Continuous Dynamical Systems-Series B 20 (2015), no. 8, 2333-2360.

[KMV05] W D Kalies, K Mischaikow, and R C A M VanderVorst, An algorithmic approach to chain recurrence, Foundations of Computational Mathematics 5 (2005), no. 4, 409-449.

[Kod21] D E Koditschek, What is robotics? Why do we need it, and how can we get it?, Annual Review of Control, Robotics, and Autonomous Systems 4 (2021), no. (to appear), 1-37.

[KR19] M D Kvalheim and S Revzen, Existence and uniqueness of global Koopman eigenfunctions for stable fixed points and periodic orbits, arXiv preprint arXiv:1911.11996 (2019).

[Lee10] J M Lee, Introduction to topological manifolds, 2 ed., Springer Science \& Business Media, 2010.

[Lee13]

[Ler16]

[Liu05]

[Liu07a] , Introduction to smooth manifolds, 2 ed., Springer-Verlag, 2013.

E Lerman, A category of hybrid systems, arXiv preprint arXiv:1612.01950 (2016).

Z Liu, The random case of Conley's theorem, Nonlinearity 19 (2005), no. 2, 277.

1017.

[Liu07b] The random case of Conley's theorem: III. random semiflow case and Morse decomposition, Nonlinearity 20 (2007), no. 12, 2773.

$\left[\mathrm{LJS}^{+}\right.$03] J Lygeros, K H Johansson, S N Simic, J Zhang, and S S Sastry, Dynamical properties of hybrid automata, IEEE Transactions on automatic control 48 (2003), no. 1, 2-17.

[Lor64] E N Lorenz, The problem of deducing the climate from the governing equations, Tellus 16 (1964), no. 1, 1-11.

$[\mathrm{LS} 20]$

[May76]

[Mil85a]

[Mil85b] E Lerman and J Schmidt, Networks of hybrid open systems, Journal of Geometry and Physics 149 (2020), 103582. R M May, Simple mathematical models with very complicated dynamics, Nature 261 (1976), no. 5560, $459-467$. J Milnor, On the concept of attractor, Communications in Mathematical Physics 99 (1985), no. 2, $177-195$. , On the concept of attractor: Correction and remarks, Communications in Mathematical Physics 102 (1985), no. 3, 517-519.

[Mil06] J W Milnor, Attractor, Scholarpedia 1 (2006), no. 11, 1815.

[MR94] J E Marsden and T S Ratiu, Introduction to mechanics and symmetry, 1 ed., Springer-Verlag, 1994.

[Mun00] J R Munkres, Topology, 2 ed., Prentice Hall, Inc., 2000.

[MW06] R P McGehee and T Wiandt, Conley decomposition for closed relations, Journal of Difference Equations and Applications 12 (2006), no. 1, 1-47.

[Nor95] D E Norton, The fundamental theorem of dynamical systems, Commentationes Mathematicae Universitatis Carolinae 36 (1995), no. 3, 585-597.

[OA10] Yizhar Or and Aaron D Ames, Stability and completion of Zeno equilibria in Lagrangian hybrid systems, IEEE Transactions on Automatic Control 56 (2010), no. 6, 1322-1336.

[Pat11] M Patrão, Existence of complete Lyapunov functions for semiflows on separable metric spaces, Far East Journal of Dynamical Systems 17 (2011), no. 1, 49-54.

[PG09] I Poulakakis and J W Grizzle, The spring loaded inverted pendulum as the hybrid zero dynamics of an asymmetric hopper, IEEE Transactions on Automatic Control 54 (2009), no. 8, 1779-1793.

[PJdM82] J Palis Jr and W de Melo, Geometric theory of dynamical systems: an introduction, Springer-Verlag, 1982.

[PT77] J Palis and F Takens, Topological equivalence of normally hyperbolic dynamical systems, Topology 16 (1977), no. 4, 335-345. MR 0474409

[RBCG17] H Razavi, A M Bloch, C Chevallereau, and J W Grizzle, Symmetry in legged locomotion: a new method for designing stable periodic gaits, Autonomous Robots 41 (2017), no. 5, 1119-1142.

[RK15] S Revzen and M Kvalheim, Data driven models of legged locomotion, Micro-and Nanotechnology Sensors, Systems, and Applications VII, vol. 9467, International Society for Optics and Photonics, 2015, p. 94671V.

[Rob99] C Robinson, Dynamical systems: stability, symbolic dynamics, and chaos, CRC Press, 1999.

[Ryb83] K P Rybakowski, The Morse index, repeller-attractor pairs and the connection index for semiflows on noncompact spaces, Journal of Differential Equations 47 (1983), no. 1, 66-98.

[RZ85] K P Rybakowski and E Zehnder, A Morse equation in Conley's index theory for semiflows on metric spaces, Ergodic Theory and Dynamical Systems 5 (1985), no. 1, 123-143. 
[SJLS01] S N Simić, K H Johansson, J Lygeros, and S Sastry, Structural stability of hybrid systems, 2001 European Control Conference (ECC), IEEE, 2001, pp. 3858-3863.

[SJLS05] _ Towards a geometric theory of hybrid systems, Dynamics of Continuous, Discrete and Impulsive Systems Series B: Applications and Algorithms 12 (2005), no. 5-6, 649-687.

[SJSL00] S N Simić, K H Johansson, S Sastry, and J Lygeros, Towards a geometric theory of hybrid systems, International Workshop on Hybrid Systems: Computation and Control, Springer, 2000, pp. 421-436.

[SKR $\left.{ }^{+} 17\right] \quad J$ Seipel, M Kvalheim, S Revzen, M A Sharbafi, and A Seyfarth, Conceptual models of legged locomotion, Bioinspired Legged Locomotion, Elsevier, 2017, pp. 55-131.

[Sma67] S Smale, Differentiable dynamical systems, Bulletin of the American mathematical Society 73 (1967), no. 6, $747-817$.

[Son89] E D Sontag, Smooth stabilization implies coprime factorization, IEEE Transactions on Automatic Control 34 (1989), no. 4, 435-443.

[SSJL02] S N Simić, S Sastry, K H Johansson, and J Lygeros, Hybrid limit cycles and hybrid Poincaré-Bendixson, IFAC Proceedings Volumes 35 (2002), no. 1, 197-202.

[Sto56] A H Stone, Metrizability of decomposition spaces, Proceedings of the American Mathematical Society 7 (1956), no. 4, 690-700.

[Tu10] L W Tu, Introduction to manifolds, 2 ed., Springer Science \& Business Media, 2010.

[Tu17] L Tu, Can a topological manifold be non-connected and each component with different dimension?, Mathematics Stack Exchange, 2017, URL:https://math.stackexchange.com/q/3208774 (version: 2019-04-30).

[TVDK19] T T Topping, V Vasilopoulos, A De, and D E Koditschek, Composition of templates for transitional pedipulation behaviors, The International Symposium on Robotics Research, 2019.

[WGK03] E R Westervelt, J W Grizzle, and D E Koditschek, Hybrid zero dynamics of planar biped walkers, IEEE transactions on automatic control 48 (2003), no. 1, 42-56.

[Wia08] T Wiandt, Liapunov functions for closed relations, Journal of Difference Equations and Applications 14 (2008), no. $7,705-722$.

[YMH98] H Ye, A N Michel, and L Hou, Stability theory for hybrid dynamical systems, IEEE transactions on automatic control 43 (1998), no. 4, 461-474. 


\section{Supplementary Materials (SM)}

\section{Appendix A. Relationship With SELECTED PRIOR WORK}

A.1. Relationship of Definition 1 to [JBK16]. Our definition of THS strictly generalizes [JBK16, Def. 2], modulo our added regularizing assumption requiring that the union of guard sets be closed. Since any disjoint union of smooth (paracompact) manifolds with corners is metrizable, our definition of MHS similarly strictly generalizes [JBK16, Def. 2], modulo the closed guard assumption and the choice of a compatible extended metric on state space. However, we note that our Theorems 1 and 2 impose two additional conditions on MHS that are not assumed in [JBK16]: our theorems require that (i) state space is compact (Assumption 4), and (ii) the trapping guard condition is satisfied (Assumption 3). (We also add that [JBK16] consider continuations of Zeno executions past the stop time, while we do not; cf. Remark 2.) Regarding (i) we note that, as discussed in Remark 5, the specific choice of compatible extended metric is immaterial for the majority of our purposes since Theorems 1 and 2 require that state space is compact. ${ }^{20}$

Regarding (ii), the hybrid systems model of [JBK16] allows for the possibility that no hybrid suspension semiflow (Def. 14) exists which is continuous-in-state, thereby precluding the trapping guard condition as shown in Appendix $\mathrm{C}$ which, in turn, may compromise the necessity of a Conley decomposition and Lyapunov function (e.g., see Ex. 3 and 4 for one view of the gap between the sufficiency and the necessity of this condition). For other classes of physical models, continuity can fail for different reasons. While our Def. 1 and [JBK16, Def. 2] require reset maps to be continuous, parsimonious hybrid models of certain physical systems may fail even to have this property (though in many applications it might be acceptable to insure continuity - e.g., one might smooth down the model of an exterior wall's outer corner so as to insure that balls bounce off it in a continuous manner). However, the discontinuities of behavior allowed by the [JBK16] and other hybrid systems models may play a key role in other problem settings, such as legged leaping as explored in [BDJK15, Fig. 7, Sec. III.b], [JK13]. Clearly, more theoretical work is needed to understand the prospect for achieving Conley-style results in these settings, while, at the same time, more empirical work is needed to understand how the phenomena of interest should be formally represented and intuitively understood in their absence.

A.2. Relationship of Definition 1 to [AS05]. Our definition of THS is particularly similar to the definition of "classical hybrid system" in [AS05, p. 92]. However, there are some differences. First, we ignore any underlying graph structure of the hybrid system, although Remark 1 explains that this is immaterial. Second, the definition in $[\mathrm{AS} 05$, p. 92] amounts, using our notation, to requiring a flow $\Phi$ be defined on $\mathcal{X}$; in contrast, we only require a semiflow be defined on $\mathcal{F} \subseteq \mathcal{X}$. Finally, we impose the regularizing requirement that the guard $\mathcal{G} \subseteq \mathcal{X}$ be closed; this requirement is not made in [AS05, p. 92].

A.3. Relationship of Definition 4 to [CGKS19]. Our definition of $(\epsilon, T)$-chains (Def. 4$)$ is closest to that of [CGKS19, Def. 2.18]. While our presentations differ, the only mathematical difference is our requirement that an $(\epsilon, T)$-chain contain at least two arcs. If this were not the case, then the Conley relation (as defined in Def. 5) would be reflexive, which would imply that every point is chain recurrent. It is clear that every $(\epsilon, T)$-chain in our sense is also an $(\epsilon, T)$-chain in the sense of [CGKS19, Def. 2.18], but not vice versa.

\section{A.4. Relationship of the relaxed hybrid system and hybrid suspension to prior work.}

A.4.1. Generalized hybrifolds. The hybrifold of a hybrid system was introduced in [SJSL00, SJLS05] for a class of hybrid systems satisfying various smoothness assumptions: e.g., state space is required to be a disjoint union of manifolds with "piecewise-smooth boundary," and reset maps are required to be diffeomorphisms onto their images. Our classes THS and MHS of hybrid systems do not assume any such smoothness nor injectivity properties, but we can still give a definition analogous to that of the hybrifold

\footnotetext{
${ }^{20}$ For the interested reader, we briefly mention that specific metrizations of hybrid systems are discussed in [BGV $\left.{ }^{+} 15\right]$. However, we caution that, e.g., the pseudometric defined in $\left[\mathrm{BGV}^{+} 15\right.$, Sec. III.A] is not generally an extended metric compatible with the topology on state space (under certain assumptions it defines a metric on a certain quotient of state space, the hybrifold discussed in §A.4.1), so it is not generally an admissible extended metric making a metrizable THS into an MHS.
} 
in our setting. We will refer to this analogous, but (formally) more general, construction as the generalized hybrifold. ${ }^{21}$

Let $H:=(\mathcal{X}, \mathcal{F}, \mathcal{G}, \varphi, r)$ be a THS. Using the notation " $M_{H}$ " of [SJSL00, SJLS05], we define the generalized hybrifold $M_{H}$ of $H$ to be the topological space obtained by gluing points $z \in \mathcal{G} \subseteq \mathcal{X}$ to $\mathcal{X}$ along the reset $r$ :

$$
M_{H}:=\mathcal{X} /(z \sim r(z)) .
$$

Assuming there exists a unique semiflow $\Psi_{M_{H}}$ on $M_{H}$ such that the quotient $\pi_{M_{H}}: \mathcal{X} \rightarrow M_{H}$ sends $H$ executions into $\Psi_{M_{H}}$ trajectories while preserving time, we refer to $\Psi_{M_{H}}$ as the generalized hybrifold semiflow. Using Lemma 1 and the universal property of the quotient topology [Lee10, Thm 3.70], it can be shown (by mimicking the proof of Prop. 5) that $\Psi_{M_{H}}$ is a well-defined and continuous semiflow if, e.g., Assumptions 1, 2, and 3 are satisfied and if there are no Zeno executions. ${ }^{22}$ A cartoon depiction of a generalized hybrifold (including a trajectory of $\Psi_{M_{H}}$ ) is shown in the bottom-left panel of Fig. 8 .

In certain situations we can prove that $M_{H}$ (with the quotient topology) is metrizable. In particular, Lemma 2 implies that, if $\mathcal{X}$ is metrizable and the reset $r$ is a closed map with compact fibers satisfying $r(\mathcal{G}) \cap \mathcal{G}=\varnothing$, then $M_{H}$ is metrizable. In particular, if $\mathcal{X}$ is compact and metrizable and $r(\mathcal{G}) \cap \mathcal{G}=\varnothing$, then $M_{H}$ is metrizable.

However, at least if $r(\mathcal{G}) \cap \mathcal{G} \neq \varnothing$, the generalized hybrifold $M_{H}$ of a compact MHS cannot be used to prove Theorems 1 and 2 for multiple reasons even if $M_{H}$ happened to be metrizable. For example (as pointed out in Remarks 15 and 17), $\omega$-limit sets, attracting-repelling pairs, and chain recurrence for $\left(M_{H}, \Psi_{H}\right)$ are not generally compatible with the corresponding notions for $H$ if $r(\mathcal{G}) \cap \mathcal{G} \neq \varnothing$.

Furthermore, even if these compatibility issues were not present for a specific MHS $H$, a complete Lyapunov function for $\Psi_{M_{H}}$ will not generally pull back to a complete Lyapunov function for $H$. More explicitly, if $V: M_{H} \rightarrow \mathbb{R}$ is a complete Lyapunov function for $\Psi_{M_{H}}$, then the function $L:=V \circ \pi_{M_{H}}$ will not generally be a complete Lyapunov function for $H$, because it will not satisfy the second condition of Def. 12 ( $L$ will not decrease across resets). Thus, the technique used in the proof of Theorem 2 would still fail if the hybrifold was used instead of the hybrid suspension.

A.4.2. Relaxed hybrid system. As mentioned in $§ 6.1$, the relaxed hybrid system $H^{\prime}$ (Def. 13) formalizes the idea of requiring that executions of the hybrid system $H$ "wait" one time unit after impacting the guard before resetting. A cartoon depicting the relaxed system is shown in the top-right panel of Fig. 8. The relaxed system is essentially an example of a "temporal relaxation" in the sense of [JELS99], where it was used to regularize Zeno executions, although we give the definition for THS which are (formally speaking) more general ${ }^{23}$ than the specific examples considered in [JELS99, Sec. 3-4] (e.g., the local semiflows for THS are not assumed to be generated by vector fields and, furthermore, the state space of a THS is a general topological space rather than any sort of manifold). While we recover this Zeno regularization in our setting (Remark 12), our primary motivation for the relaxed system is to use it as an intermediate step in constructing the hybrid suspension (Def. 14), which has better properties than those of the generalized hybrifold discussed in §A.4.1.

\footnotetext{
${ }^{21}$ The terminology hybrifold is unfortunately no longer appropriate since no manifolds are involved. As one possible alternative, this generalization has also been referred to as a "colimit" in [AS05, p. 94].

${ }^{22}$ We emphasize that these conditions - in particular, the trapping guard condition - are only sufficient to ensure that a well-defined and continuous generalized hybrifold semiflow exists. The simple example of a THS $H=(\mathcal{X}, \mathcal{F}, \mathcal{G}, \varphi, r)$ with $\mathcal{X}=[0,1], \mathcal{F}=(0,1], \mathcal{G}=\{0\}, r(0)=1$, and $\varphi$ generated by the vector field $-x(1-x) \frac{\partial}{\partial x}$ shows that the trapping guard is not necessary for the generalized hybrifold semiflow $\Psi_{M_{H}}$ to be well-defined and continuous. The reader may wish to contrast this with the converse statement of Cor. 5 in Appendix C for the hybrid suspension semiflow $\Phi_{H}$.

${ }^{23}$ Engineers might be unimpressed by the apparently slight formal gain of generality. Applications generally present models with smooth manifolds carrying (at least piecewise) smooth vector fields. In contrast, classical Conley theory is rooted in the tools of topological dynamics whose framework we have thus found it natural to adopt here. Furthermore, we hope that the imperative to eliminate smoothness assumptions from the spaces carrying these dynamics may be intuitively apparent when considering the (pinched and creased non-manifold) topological spaces that inevitably arise as depicted, for example, in the hybrid suspension $\Sigma_{H}$ of Fig. 6 .
} 

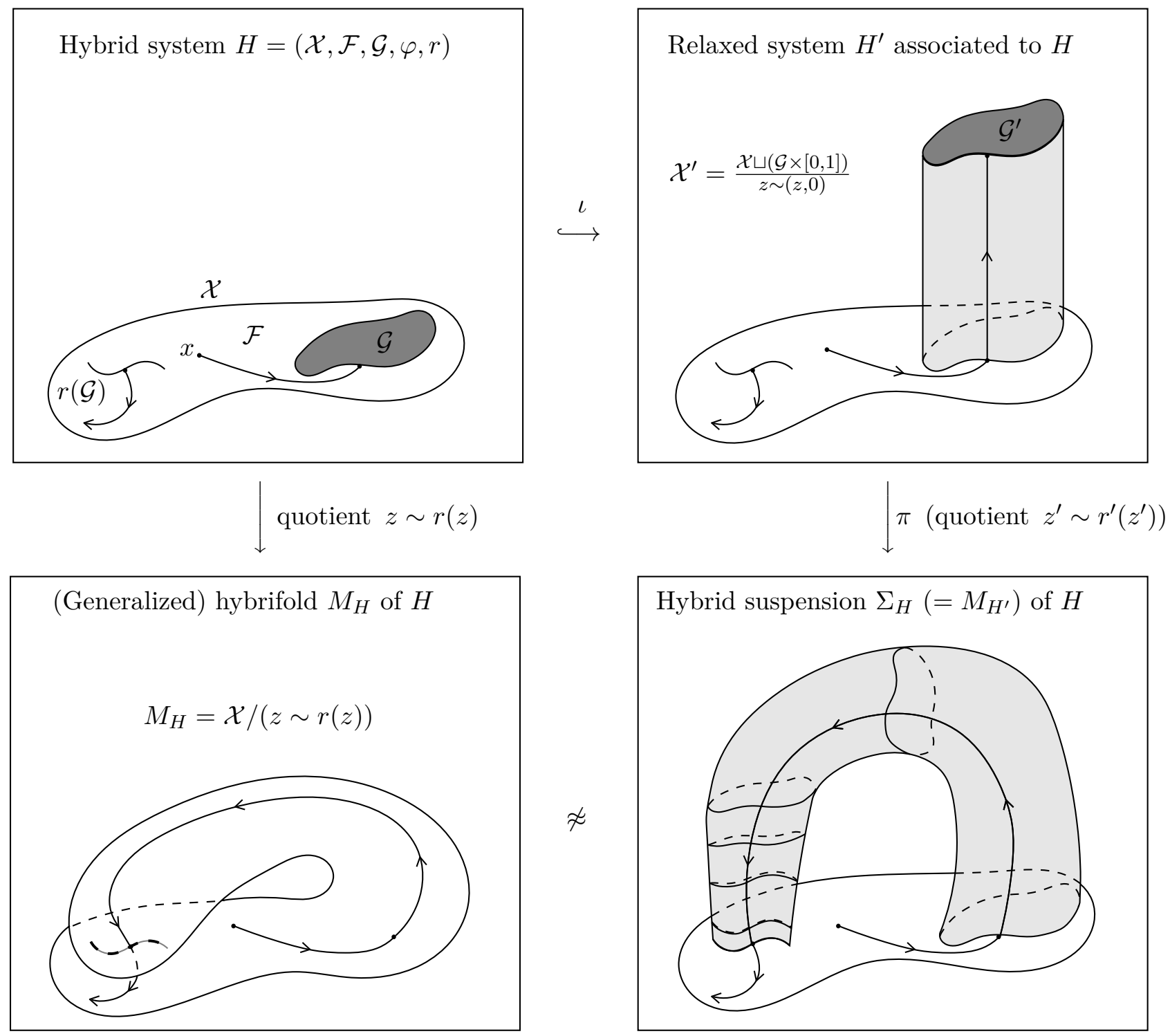

Figure 8. Comparison of the constructions from $\S 6.1$ depicted in Fig. 6 with the generalized hybrifold $M_{H}$ of $H$ discussed in $\S$ A.4.1. Top left: a THS $H=(\mathcal{X}, \mathcal{F}, \mathcal{G}, \varphi, r)$. Top right: its relaxed version $H^{\prime}=\left(\mathcal{X}^{\prime}, \mathcal{F}^{\prime}, \mathcal{G}^{\prime}, \varphi^{\prime}, r^{\prime}\right)$; see Def. 13. Bottom right: hybrid suspension $\left(\Sigma_{H}, \Phi_{H}\right)$ of $H$; see Def. 14. Bottom left: the generalized hybrifold $M_{H}$ of $H ; M_{H}$ is formed by gluing $\mathcal{G}$ directly to $r(\mathcal{G})$ along $r$, without first embedding $\mathcal{X}$ in a larger space (unlike $\left.\Sigma_{H}=M_{H^{\prime}}\right)$. We mention $M_{H}$ only for purposes of comparison, i.e., we do not use $M_{H}$ in this paper. We remark that $\Sigma_{H}$ coincides with the generalized hybrifold $M_{H^{\prime}}$ of $H^{\prime}$ (but not with $\left.M_{H}\right)$. Additionally, we emphasize that $\left(\Sigma_{H}, \Phi_{H}\right)$ strictly generalizes the classical suspension of a discrete-time dynamical system discussed in Appendix B; indeed, if $\mathcal{X}=\mathcal{G}$ (cf. Ex. 1) our construction reduces to the classical one.

A.4.3. Prior work related to the hybrid suspension. The technique we used to prove Theorems 1 and 2 involves showing that a THS satisfying the trapping guard condition and certain other assumptions is, in a certain sense (Prop.s 6, 7, and 8), no different from a certain continuous-time (semi-)dynamical system. As discussed in §A.4.1 and Remarks 15 and 17, this continuous-time system is not the generalized hybrifold (local) semiflow; it is the hybrid suspension semiflow constructed in Def. 14 . We choose to use the terminology "suspension" because the hybrid suspension strictly generalizes the classical suspension 
[Sma67, BS02, p. 797, pp. 21-22] of a discrete-time dynamical system; indeed, if $\mathcal{X}=\mathcal{G}$ our construction reduces to the classical one (as explained in Appendix B). We give a brief primer on the classical suspension in Appendix B.

In writing this paper we have become aware that versions of the hybrid suspension have previously appeared in the literature under different names, although (to the best of our knowledge) only for classes of hybrid systems which are not as general as THS. As mentioned in $\S 1.2$, the hybrid suspension $\Sigma_{H}$ of a THS $H$ could be called a "1-relaxed hybrid quotient space" in the terminology of $\left[\mathrm{BGV}^{+} 15\right]$ or a "homotopy colimit" in the terminology of [AS05].

We finally note that, in the terminology introduced in $\S$ A.4.1, the hybrid suspension $\Sigma_{H}$ of a THS $H$ coincides with the generalized hybrifold $M_{H^{\prime}}$ (where $H^{\prime}$ is the relaxed hybrid system) but not with the hybrifold $M_{H}$. See Fig. 8 .

\section{Appendix B. Classical suspension of a Discrete-time dynamical System}

The purpose of this appendix is to explain, in a self-contained way, (i) the classical suspension of a discrete-time dynamical system and (ii) how the hybrid suspension (Def. 14) strictly generalizes the classical notion.

The classical suspension is often considered in the context of a $C^{r \geq 1}$ diffeomorphism of a $C^{r}$ manifold [Sma67, PT77, PJdM82, Rob99, p. 797, pp. 343-345, p. 111, p. 173]. However, relevant for us is the more general context of a discrete-time (semi-)dynamical system defined by a continuous map of a topological space; we now describe the classical suspension in this context, following roughly [BS02, pp. 21-22].

Let $X$ be a topological space, $f: X \rightarrow X$ be a continuous map defining a discrete-time (semi-)dynamical system $(n, x) \mapsto f^{\circ n}(x)$, and $c: X \rightarrow(0, \infty)$ be a continuous function bounded away from zero. Consider the quotient space

$$
X_{c}:=\{(x, t) \in X \times[0, \infty): 0 \leq t \leq c(x)\} / \sim,
$$

where $\sim$ is the equivalence relation $(x, c(x)) \sim(f(x), 0) . X_{c}$ is called the suspension with ceiling (or roof) function $c$. Letting $[(x, s)] \in X_{c}$ denote the equivalence class of $(x, s)$, the suspension semiflow (with ceiling function $c$ ) is the semiflow $\phi_{c}:[0, \infty) \times X_{c} \rightarrow X_{c}$ given by $\phi^{t}([x, s])=\left[\left(f^{\circ n}(x), s^{\prime}\right)\right]$, where $n \in \mathbb{N}$ and $s^{\prime} \geq 0$ satisfy

$$
\sum_{i=0}^{n-1} c\left(f^{\circ i}(x)\right)+s^{\prime}=t+s, \quad 0 \leq s^{\prime} \leq c\left(f^{\circ n}(x)\right) .
$$

It is common to simply take the ceiling function $c$ to be $c(x) \equiv 1$ [Sma67, Rob99, PT77, PJdM82, Rob99, p. 797 , pp. $343-345$, p. 111 , p. 173], and in this case it is common to simply refer to $\left(X_{1}, \phi_{1}\right)$ as "the" suspension of (the discrete-time semi-dynamical system defined by) $f{ }^{24}$ Our hybrid suspension defined in Def. 14 strictly generalizes "the" suspension of $f$, and this can be seen as follows. Define a THS $H=$ $(\mathcal{X}, \mathcal{F}, \mathcal{G}, \varphi, r)$ by setting $\mathcal{X}=\mathcal{G}=X, \mathcal{F}=\varnothing, r=f$, and (viewed set-theoretically) $\varphi=\varnothing$ (cf. Ex. 1). Then the hybrid suspension $\left(\Sigma_{H}, \Phi_{H}\right)$ coincides precisely with $\left(X_{1}, \phi_{1}\right)$.

Appendix C. Continuous hybrid Suspension SEMiflow implies the trapping Guard CONDition

Let $H=(\mathcal{X}, \mathcal{F}, \mathcal{G}, \varphi, r)$ be a THS satisfying Assumptions 1, 2, and 3. In $\S 6.1$ (Def. 13 and 14) we defined

$$
\begin{gathered}
\mathcal{X}^{\prime}:=\frac{\mathcal{X} \sqcup(\mathcal{G} \times[0,1])}{z \sim(z, 0)} \quad \underbrace{\pi_{0}: \mathcal{X} \sqcup(\mathcal{G} \times[0,1]) \rightarrow \mathcal{X}^{\prime}}_{\text {quotient map }} \quad \underbrace{\iota: \mathcal{X} \rightarrow \mathcal{X}^{\prime}}_{\left.\pi_{0}\right|_{\mathcal{X}}} \\
\Sigma_{H}:=\frac{\mathcal{X}^{\prime}}{\pi_{0}(z, 1) \sim \pi_{0}(r(z))} \quad \underbrace{\pi: \mathcal{X}^{\prime} \rightarrow \Sigma_{H}}_{\text {quotient map }}
\end{gathered}
$$

and the suspension semiflow $\Phi_{H}:[0, \infty) \times \Sigma_{H} \rightarrow \Sigma_{H}$, and we showed that $\Phi_{H}$ is continuous (Prop. 5). It is immediate from the definitions that $\Phi_{H}$ satisfies the following two properties.

\footnotetext{
${ }^{24}$ We note that $X_{1}$ coincides with what topologists call the mapping torus of $f$ [Hat01, p. 53, p. 151] (but, confusingly, not with what topologists call the suspension of a topological space [Hat01, p. 8]).
} 
1. $\Phi_{H}^{t}\left(\pi \circ \pi_{0}(z, s)\right)=\pi \circ \pi_{0}(z, t+s)$ for all $z \in \mathcal{G}, s \in[0,1]$, and $t \in[0,1-s]$.

2. For all $(t, x) \in \operatorname{dom}(\varphi), \pi \circ \iota\left(\varphi^{t}(x)\right)=\Phi_{H}^{t}(\pi \circ \iota(x))$.

While for convenience of exposition we only defined the quantities in (24) under Assumptions 1, 2, and 3 (in particular, under Assumption 3), the definitions in (24) make sense verbatim for any THS. Thus, for an arbitrary THS $H$, it makes sense to ask the following question: under what circumstances does there exist a well-defined "suspension semiflow" $\Phi$ on $\Sigma_{H}$ for $H$ in the sense that $\Phi$ satisfies Conditions 1 and 2 (stated above for $\left.\Phi_{H}\right)$ ?

In this appendix we prove a result (Prop. 9) which implies (Cor. 5) that, if $H=(\mathcal{X}, \mathcal{F}, \mathcal{G}, \varphi, r)$ is a THS satisfying Assumptions 1 and 2 with Hausdorff $\mathcal{X}$, then there exists a continuous suspension semiflow $\Phi:[0, \infty) \times \Sigma_{H} \rightarrow \Sigma_{H}$ in the above sense if and only if $H$ satisfies the trapping guard condition (Assumption 3).

We state Prop. 9 after first establishing the following preliminary result.

Lemma 7. Let $H=(\mathcal{X}, \mathcal{F}, \mathcal{G}, \varphi, r)$ be a THS. Define $\mathcal{X}^{\prime}, \Sigma_{H}, \pi_{0}, \iota$, and $\pi$ as in (24). Then

$$
\left.\pi \circ \pi_{0}\right|_{\mathcal{G} \times\left[0, \frac{1}{2}\right]}: \mathcal{G} \times\left[0, \frac{1}{2}\right] \rightarrow \Sigma_{H} \quad \text { and } \quad \pi \circ \iota: \mathcal{X} \rightarrow \Sigma_{H}
$$

are homeomorphisms onto their images.

Proof. We first show that $\left.\pi\right|_{\iota(\mathcal{X}) \cup \pi_{0}\left(\mathcal{G} \times\left[0, \frac{1}{2}\right]\right)}$ is a closed map. Define $\mathcal{G}^{\prime}:=\pi_{0}(\mathcal{G} \times\{1\})$ and $r^{\prime}: \mathcal{G}^{\prime} \rightarrow \mathcal{X}^{\prime}$ via $r^{\prime}\left(\pi_{0}(z, 1)\right):=\pi_{0}(r(z))$, and let $C \subseteq \iota(\mathcal{X}) \cup \pi_{0}\left(\mathcal{G} \times\left[0, \frac{1}{2}\right]\right)$ be an arbitrary closed set. We compute

$$
\pi^{-1}(\pi(C))=C \cup\left(r^{\prime}\right)^{-1}(C) \cup r^{\prime}(\underbrace{C \cap \mathcal{G}^{\prime}}_{=\varnothing})
$$

since $r^{\prime}\left(\mathcal{G}^{\prime}\right) \cap \mathcal{G}^{\prime}=\varnothing$. Since $r^{\prime}$ is continuous, the right side of (25) is the union of three closed sets (the third is empty since $\iota(\mathcal{X}) \cup \pi_{0}\left(\mathcal{G} \times\left[0, \frac{1}{2}\right]\right)$ is disjoint from $\left.\mathcal{G}^{\prime}\right)$. By the definition of the quotient topology, it follows that $\pi(C)$ is closed in $\Sigma_{H}$, so $\left.\pi\right|_{\iota(\mathcal{X}) \cup \pi_{0}\left(\mathcal{G} \times\left[0, \frac{1}{2}\right]\right)}$ is indeed a closed map.

Since $\left.\pi\right|_{\iota(\mathcal{X}) \cup \pi_{0}\left(\mathcal{G} \times\left[0, \frac{1}{2}\right]\right)}$ is a closed map,

$$
\left.\pi \circ \pi_{0}\right|_{\mathcal{G} \times\left[0, \frac{1}{2}\right]}=\left.\left.\pi\right|_{\iota(\mathcal{X}) \cup \pi_{0}\left(\mathcal{G} \times\left[0, \frac{1}{2}\right]\right)} \circ \pi_{0}\right|_{\mathcal{G} \times\left[0, \frac{1}{2}\right]} \quad \text { and } \quad \pi \circ \iota=\left.\pi\right|_{\iota(\mathcal{X}) \cup \pi_{0}\left(\mathcal{G} \times\left[0, \frac{1}{2}\right]\right)} \circ \iota
$$

are closed maps by composition, since $\pi_{0}$ and $\iota$ are closed maps. That $\pi_{0}$ is closed follows by repeating the proof of Lemma 3 verbatim, and $\iota$ is closed since $\pi_{0}^{-1}(\iota(D))=D \sqcup((D \cap \mathcal{G}) \times\{0\})$ is closed in $\mathcal{X} \sqcup(\mathcal{G} \times[0,1])$ for any closed set $D \subseteq \mathcal{X}$.

It is immediate from the definitions that both maps in the statement of the lemma are continuous and injective. Since we have shown that they are also closed, it follows that they are homeomorphisms onto their images [Lee10, Ex. 2.29].

Proposition 9. Let $H=(\mathcal{X}, \mathcal{F}, \mathcal{G}, \varphi, r)$ be a THS satisfying Assumption 1 with Hausdorff $\mathcal{X}$ and with $\mathcal{X}=\mathcal{F} \cup \mathcal{G}$. Define $\mathcal{X}^{\prime}, \Sigma_{H}, \pi_{0}, \iota$, and $\pi$ as in (24), and suppose there exists a continuous semiflow $\Phi:[0, \infty) \times \Sigma_{H} \rightarrow \Sigma_{H}$ satisfying the following conditions.

9.1. $\Phi^{t}\left(\pi \circ \pi_{0}(z, s)\right)=\pi \circ \pi_{0}(z, t+s)$ for all $z \in \mathcal{G}, s \in[0,1]$, and $t \in[0,1-s]$.

9.2. For all $(t, x) \in \operatorname{dom}(\varphi), \pi \circ \iota\left(\varphi^{t}(x)\right)=\Phi^{t}(\pi \circ \iota(x))$.

Then $H$ satisfies the trapping guard condition (Assumption 3).

Corollary 5. Let $H=(\mathcal{X}, \mathcal{F}, \mathcal{G}, \varphi, r)$ be a THS satisfying Assumptions 1 and 2 with Hausdorff $\mathcal{X}$. Define $\mathcal{X}^{\prime}, \Sigma_{H}, \pi_{0}, \iota$, and $\pi$ as in (24). Then there exists a continuous "suspension semiflow" $\Phi:[0, \infty) \times \Sigma_{H} \rightarrow \Sigma_{H}$ for $H$-in the sense that $\Phi$ satisfies conditions 9.1 and 9.2 of Prop. 9-if and only if $H$ satisfies the trapping guard condition (Assumption 3).

Proof of Cor. 5. If $H$ satisfies the trapping guard condition, then by Prop. 5 the map $\Phi_{H}$ of Def. 14 is such a continuous semiflow.

Conversely, assume that a continuous semiflow $\Phi$ satisfying Conditions 9.1 and 9.2 exists. The assumption that all maximal executions are infinite or Zeno (Assumption 2) implies that $\mathcal{X}=\mathcal{F} \cup \mathcal{G}$ (by Remark 3), so the hypotheses of Prop. 9 are satisfied. By the conclusion of Prop. 9, $H$ satisfies the trapping guard condition. 
Proof of Prop. 9. For purposes of readability, we define $f:=\pi \circ \iota: \mathcal{X} \rightarrow \pi \circ \iota(\mathcal{X}), B:=\mathcal{G} \times\left[0, \frac{1}{2}\right]$, and $g:=\left.\left(\pi \circ \pi_{0}\right)\right|_{B}: B \rightarrow \pi \circ \pi_{0}(B)$. By Lemma $7, f$ and $g$ are homeomorphisms.

Letting $\mu: \mathcal{X} \rightarrow[0,+\infty]$ be the maximum flow time defined in (1), we first show that, for any $x \in$ $\mathcal{F} \cap \mu^{-1}([0, \infty))$,

$$
\ell(x):=\lim _{t \rightarrow \mu(x)^{-}} \varphi^{t}(x) \in \mathcal{G} .
$$

That the limit $\ell(x)$ exists follows from continuity of $f, f^{-1}$, and $\Phi$ since Condition 9.2 implies that $\varphi^{t}(x)=f^{-1}\left(\Phi^{t}(f(x))\right)$ for all $t \in\{t \mid(t, x) \in \operatorname{dom}(\varphi)\}$, and the properties of a local semiflow imply that $\{t \mid(t, x) \in \operatorname{dom}(\varphi)\}=[0, \mu(x))$ for any $x \in \mathcal{F}$ [HS06, Sec. 1.3]. Furthermore, it cannot be the case that $\ell(x) \in \mathcal{F}$, because the trajectory image $\varphi^{[0, \mu(x))}(x)$ would then have compact closure in $\mathcal{F}$, and this in turn would imply that $\mu(x)$ is infinite [HS06, Sec. 1.3], a contradiction. Since we have also assumed that $\mathcal{X}=\mathcal{F} \cup \mathcal{G}$, it follows that $\ell(x) \in \mathcal{G}$.

Next, define $\widetilde{U}:=\Phi^{-\frac{1}{2}}(g(B)), U:=f^{-1}(\widetilde{U})$, and the continuous maps $h: g(B) \rightarrow\left[0, \frac{1}{2}\right]$ and $\nu: U \rightarrow\left[0, \frac{1}{2}\right]$ via $h(g(z, t)):=t$ and $\nu:=\frac{1}{2}-\left.h \circ \Phi^{\frac{1}{2}} \circ f\right|_{U}$. By the definition of $\nu$ and Condition 9.1 it follows that $\nu^{-1}(0)=\mathcal{G}$ and $\Phi^{\nu(x)}(f(x)) \in f(\mathcal{G})$ for all $x \in U$. We will now show that $\left.\mu\right|_{U}=\nu$. Since $\varphi$ is $\mathcal{F}$-valued but $\Phi^{\nu(x)}(f(x)) \in f(\mathcal{G})$, it follows from Condition 9.2 and the fact that $\mathcal{F} \cap \mathcal{G}=\varnothing$ (since $H$ is deterministic by Assumption 1) that $(\nu(x), x) \notin \operatorname{dom}(\varphi)$ for any $x \in U$. Since $\{t \mid(t, x) \in \operatorname{dom}(\varphi)\}=[0, \mu(x))$ for any $x \in \mathcal{F}$ [HS06, Sec. 1.3], it follows that $\left.\mu\right|_{U \cap \mathcal{F}} \leq\left.\nu\right|_{U \cap \mathcal{F}}$, and therefore $\left.\mu\right|_{U} \leq \nu$ since $\left.\mu\right|_{\mathcal{G}}=\left.\nu\right|_{\mathcal{G}}=0$. We now show the reverse inequality. It follows from 9.1 that, if $q \in f(\mathcal{G})$, then $\Phi^{t}(q) \notin f(\mathcal{G})$ for all $t \in(0,1)$. Since $\nu \leq \frac{1}{2}$ and $\Phi^{\nu(x)}(f(x)) \in \mathcal{G}$ for all $x \in U$, it therefore follows that $\Phi^{t}(f(x)) \notin f(\mathcal{G})$ for all $t \in[0, \nu(x))$. Therefore, 9.2 implies that $\lim _{s \rightarrow t^{-}} \varphi^{s}(x)=f^{-1}\left(\Phi^{t}(f(x))\right) \notin \mathcal{G}$ for any $x \in U$ and $t \in[0, \nu(x))$. From this and (26) it follows that $\left.\mu\right|_{U} \geq \nu$. Since we have already shown that $\left.\mu\right|_{U} \leq \nu$, this establishes that $\left.\mu\right|_{U}=\nu$.

Since $\widetilde{U} \cap f(\mathcal{X})=\Phi^{-\frac{1}{2}}(g(B)) \cap f(\mathcal{X})=\Phi^{-\frac{1}{2}}(g(\mathcal{G} \times[0,1])) \cap f(\mathcal{X})$ is a neighborhood of $f(\mathcal{G})$ in $f(\mathcal{X})$, $U=f^{-1}(\widetilde{U})$ is a neighborhood of $\mathcal{G}$ in $\mathcal{X}$. We now define $\widehat{\varphi}: \operatorname{cl}(\operatorname{dom}(\varphi)) \cap([0, \infty) \times U) \rightarrow \mathcal{X}$ and $\rho: U \rightarrow \mathcal{G}$ via

$$
\widehat{\varphi}^{t}(x):=f^{-1} \circ \Phi^{t} \circ f(x), \quad \rho(x):=\widehat{\varphi}^{\nu(x)}(x) .
$$

Condition 9.2 implies that $\widehat{\varphi}$ is a continuous extension of $\left.\varphi\right|_{\operatorname{dom}(\varphi) \cap([0, \infty) \times U)}$ which satisfies Equation (2) of Def. 11 since $\nu=\left.\mu\right|_{U}$, and this extension is automatically unique since $\mathcal{X}$ is Hausdorff. The map $\rho$ is a continuous retraction by (27) and the fact that $\Phi^{\nu(x)}(f(x)) \in f(\mathcal{G})$ for all $x \in U$ (as noted in the previous paragraph). Since $\left.\mu\right|_{U}=\nu$ is continuous, it follows that all conditions of Def. 11 are satisfied. Hence $H$ satisfies the trapping guard condition.

\section{Appendix D. Proofs of Lemmas 1, 4, 5, And 6}

In this appendix we prove Lemmas 1, 4, 5, and 6; we also restate these lemmas for convenience.

Lemma 1. Let $H=(\mathcal{X}, \mathcal{F}, \mathcal{G}, \varphi, r)$ be a THS satisfying Assumptions 1,2 , and 3. Then $\mu: \mathcal{X} \rightarrow[0,+\infty]$ is continuous, the closure $\operatorname{cl}(\operatorname{dom}(\varphi))$ of $\operatorname{dom}(\varphi)$ in $[0, \infty) \times \mathcal{X}$ satisfies

$$
\operatorname{cl}(\operatorname{dom}(\varphi))=\{(t, x) \in[0, \infty) \times \operatorname{cl}(\mathcal{F}) \mid t \leq \mu(x)\}
$$

and $\varphi$ has a unique continuous extension $\widetilde{\varphi}$ defined on $\operatorname{cl}(\operatorname{dom}(\varphi))$ satisfying $\widetilde{\varphi}^{\mu(x)}(x) \in \mathcal{G}$ for all $x \in$ $\operatorname{cl}(\mathcal{F}) \cap \mu^{-1}([0, \infty))$.

Furthermore, $\widetilde{\varphi}$ satisfies the following conditions, with $t, s \in[0, \infty):$ (i) $\widetilde{\varphi}^{0}=\operatorname{id}_{\mathrm{cl}(\mathcal{F})}$, (ii) $(t+s, x) \in$ $\operatorname{cl}(\operatorname{dom}(\varphi)) \Longleftrightarrow \operatorname{both}(s, x) \in \operatorname{cl}(\operatorname{dom}(\varphi))$ and $\left(t, \widetilde{\varphi}^{s}(x)\right) \in \operatorname{cl}(\operatorname{dom}(\varphi))$, and (iii) for all $(t+s, x) \in$ $\operatorname{cl}(\operatorname{dom}(\varphi)), \widetilde{\varphi}^{t+s}(x)=\widetilde{\varphi}^{t}\left(\widetilde{\varphi}^{s}(x)\right)$.

Proof. We first show that $\mu$ is continuous. Letting $U \supseteq \mathcal{G}$ be the domain of a flow-induced retraction, Assumption 3 and the definition of the trapping guard condition (Def. 11) imply that $\left.\mu\right|_{U}$ is continuous. Since there is an infinite or Zeno execution starting at every $x \in \mathcal{X}$ (by Assumption 2$), \mu^{-1}([0, \infty))=$ 
$U \cup \bigcup_{t \geq 0}\left(\varphi^{t}\right)^{-1}(U)$ is a union of open subsets of $\mathcal{X} .{ }^{25}$ Since the restrictions $\left.\mu\right|_{U}$ and $\left.\mu\right|_{\left(\varphi^{t}\right)^{-1}(U)}=t+\left.\mu\right|_{U} \circ$ $\left.\varphi^{t}\right|_{\left(\varphi^{t}\right)^{-1}(U)}$ are all continuous, $\mu$ is continuous on $\mu^{-1}([0, \infty))$. Since $\operatorname{dom}(\varphi)$ is open $\operatorname{in}^{26}[0, \infty) \times \mathcal{X}$ it follows that, for every $x \in \mu^{-1}(+\infty)$ and $T>0$, there exists a neighborhood $V \ni x$ with $\mu(V) \subseteq[T,+\infty]$. Hence $\mu$ is also continuous at every point of $\mu^{-1}(+\infty)$, so $\mu: \mathcal{X} \rightarrow[0,+\infty]$ is continuous.

We now show that $\mathrm{cl}(\operatorname{dom}(\varphi))$ is given by $(7)$. Clearly $\operatorname{dom}(\varphi)$ is contained in the sets on both sides of (7). If $(t, x) \notin \operatorname{dom}(\varphi)$ belongs to the set on the right of $(7)$, then $t=\mu(x)$ since $\left.\mu\right|_{\mathcal{X} \backslash \mathcal{F}} \equiv 0$ and the properties of a local semiflow imply that $\{t \mid(t, x) \in \operatorname{dom}(\varphi)\}=[0, \mu(x))$ for all $x \in \mathcal{F}$ [HS06, Sec. 1.3]. If $\mu(x)=0$, then $(t, x) \in\{0\} \times \operatorname{cl}(\mathcal{F}) \subseteq \operatorname{cl}(\operatorname{dom}(\varphi))$ since $\{0\} \times \mathcal{F} \subseteq \operatorname{dom}(\varphi)$. If $\mu(x)>0$, then $x \in \mathcal{F}$ and $(\mu(x), x) \in \operatorname{cl}([0, \mu(x))) \times\{x\} \subseteq \operatorname{cl}(\operatorname{dom}(\varphi))$ since $[0, \mu(x)) \times\{x\} \subseteq \operatorname{dom}(\varphi)$. Hence the set on the right of (7) is contained in the set on the left. On the other hand, if $(t, x)$ does not belong to the set on the right of (7), then either (i) $t>\mu(x)$ or (ii) $x \notin \operatorname{cl}(\mathcal{F})$. In case (i), continuity of $\mu$ implies that there are neighborhoods $V \ni x$ and $J \ni t$ such that $s>\mu(y)$ for all $(s, y) \in J \times V$, so $(J \times V) \cap \operatorname{dom}(\varphi)=\varnothing$, and therefore $(t, x) \notin \operatorname{cl}(\operatorname{dom}(\varphi))$. In case (ii), $[0, \infty) \times(\mathcal{X} \backslash \operatorname{cl}(\mathcal{F}))$ is a neighborhood of $(t, x)$ disjoint from $\operatorname{dom}(\varphi)$, so again $(t, x) \notin \operatorname{cl}(\operatorname{dom}(\varphi))$. Hence the set on the left of $(7)$ is also contained in the set on the right.

From Remark 3 we have $\mathcal{X}=\mathcal{F} \cup \mathcal{G}$, and this implies that $\mu^{-1}(0)=\mathcal{G}$. Let $\widehat{\varphi}$ be the unique continuous extension of $\left.\varphi\right|_{\operatorname{dom}(\varphi) \cap([0, \infty) \times U)}$ to $\operatorname{cl}(\operatorname{dom}(\varphi)) \cap([0, \infty) \times U)$ ensured by Def. 11 . We now define $\widetilde{\varphi}: \operatorname{cl}(\operatorname{dom}(\varphi)) \rightarrow \mathcal{X}$ via

$$
\widetilde{\varphi}^{t}(x)=\left\{\begin{array}{ll}
\varphi^{t}(x), & (t, x) \in \operatorname{dom}(\varphi), \\
\widehat{\varphi}^{s}\left(\varphi^{t-s}(x)\right), & (t-s, x) \in \varphi^{-1}(U) \\
\hat{\varphi}^{t}(x), & x \in U,(t, x) \in \operatorname{cl}(\operatorname{dom}(\varphi))
\end{array},\right.
$$

where $s \in[0, t]$ ranges over all admissible values. It is clear that $\widetilde{\varphi}$ is well-defined by the definition of $\widehat{\varphi}$ and the fact that $\varphi$ satisfies the properties of a local semiflow. Since $\widetilde{\varphi}$ is defined by a family of continuous functions defined on open subsets of $\operatorname{cl}(\operatorname{dom}(\varphi))$, it follows that $\widetilde{\varphi}$ is continuous, so $\widetilde{\varphi}$ is indeed a continuous extension of $\varphi$ to $\operatorname{cl}(\operatorname{dom}(\varphi))$. Uniqueness of $\widetilde{\varphi}$ follows from uniqueness of $\widehat{\varphi}$ and the local semiflow properties of $\varphi$.

If $x \in \operatorname{cl}(\mathcal{F}) \cap \mathcal{G}$, then $\widetilde{\varphi}^{\mu(x)}(x)=x \in \mathcal{G}$ since $\mu^{-1}(0)=\mathcal{G}$. If instead $x \in \mathcal{F} \cap \mu^{-1}([0, \infty))$, then $y:=\varphi^{\mu(x)-s}(x) \in U$ for some $s \in[0, \mu(x))$, so (28) and Def. 11 imply that $\widetilde{\varphi}^{\mu(x)}(x)=\widehat{\varphi}^{s}\left(\varphi^{\mu(x)-s}(x)\right)=$ $\widehat{\varphi}^{\mu(y)}(y) \in \mathcal{G}$ since $s=\mu(y)$.

It remains only to verify the claimed properties (i-iii). (i) is immediate from (28), the definition of $\widehat{\varphi}$, and the fact that $\varphi^{0}=\mathrm{id} \mathcal{F}$. To prove (ii) first notice that, since $\left.\tilde{\varphi}\right|_{\operatorname{dom}(\varphi)}=\varphi$, the analogous property satisfied by $\operatorname{dom}(\varphi)$ is equivalent to

$$
\mu(x)=s+\mu\left(\widetilde{\varphi}^{s}(x)\right)
$$

for all $x \in \mathcal{F}$ and $s \in[0, \mu(x))$. Taking the limit as $s \rightarrow \mu(x)$ and using continuity of $\mu$ implies that (29) also holds for $x \in \mathcal{F}$ and $s \in[0, \mu(x)]$. On the other hand, (29) trivially holds for all $x \in \operatorname{cl}(\mathcal{F}) \backslash \mathcal{F}$ and $s \in[0, \mu(x)]$ since then $\mu(x)=0$ and $\widetilde{\varphi}^{0}(x)=x$. Hence (29) holds for all $x \in \operatorname{cl}(\mathcal{F})$ and $s \in[0, \mu(x)]$, and this is equivalent to the claimed property (ii). Finally, the property (iii) is trivially verified for $x \in \operatorname{cl}(\mathcal{F}) \backslash \mathcal{F}$, and is verified for $x \in \mathcal{F}$ by taking sequences $t_{n} \nearrow t, s_{n} \nearrow s$, using continuity of $\widetilde{\varphi}$, and using the analogous property satisfied by $\varphi$.

Lemma 4. Let $H=(\mathcal{X}, \mathcal{F}, \mathcal{G}, \varphi, r)$ be a MHS satisfying Assumptions $1,2,3$, and 4. Then

$$
\mathcal{C} h_{H}=\widehat{\mathcal{C} h_{H}} \text {. }
$$

In particular, two points of $\mathcal{X}$ are chain equivalent if and only if they are chain equivalent with respect to nice chains only.

\footnotetext{
${ }^{25}$ This follows since the domain of $\varphi^{t}$ is open in $\mathcal{F}$ (since $\operatorname{dom}(\varphi)$ is open in $[0, \infty) \times \mathcal{F}$, by the definition of local semiflow), and $\mathcal{F}$ is open in $\mathcal{X}$ by Def. 1 .

${ }^{26}$ This follows $\operatorname{since} \operatorname{dom}(\varphi)$ is open in $[0, \infty) \times \mathcal{F}$, and $[0, \infty) \times \mathcal{F}$ is open in $[0, \infty) \times \mathcal{X}($ since $\mathcal{F}$ is open in $\mathcal{X}$, by Def. 1$)$.
} 
Proof. It follows immediately from the definitions that $\widehat{\mathcal{C h}} \subseteq \mathcal{C} h_{H}$.

For the reverse inclusion, suppose that $(x, y) \in \mathcal{C} h_{H}$. Fix $\epsilon, T>0$, and let $U$ be a retraction domain (by Assumption 3 and Def. 11) for $\mathcal{G}$ with flow-induced retraction $\rho: U \rightarrow \mathcal{G}$. Shrinking $U$ if necessary, by Assumption 4 we may assume that $U$ is compact and that $\left.\mu\right|_{U}$ is strictly bounded above by $T$, where the maximum flow time $\mu: \mathcal{X} \rightarrow[0,+\infty]$ is defined in (1). By the uniform continuity of $r \circ \rho$, there exists $0<\delta<\epsilon$ such that $\operatorname{dist}\left(r \circ \rho(p), r \circ \rho\left(p^{\prime}\right)\right)<\epsilon / 2$ whenever $\operatorname{dist}\left(p, p^{\prime}\right)<\delta$. If $\mathcal{X} \backslash U$ is nonempty, we may further assume that $\delta<\operatorname{dist}(\mathcal{G}, \mathcal{X} \backslash U)$. By Assumptions 1, 2, and 3, Lemma 1 yields a continuous extension $\widetilde{\varphi}$ of $\varphi$ defined on the closure $\operatorname{cl}(\operatorname{dom}(\varphi))$ of $\operatorname{dom}(\varphi)$ in $[0, \infty) \times \mathcal{X}$. By compactness of $\mathcal{X}$ (Assumption 4), it follows that the restriction of $\widetilde{\varphi}$ to $\operatorname{cl}(\operatorname{dom}(\varphi)) \cap([0,2 T] \times \mathcal{X})$ is uniformly continuous. Pick $0<\beta<\epsilon / 2$ such that $\operatorname{dist}\left(\widetilde{\varphi}^{t}(p), \widetilde{\varphi}^{t^{\prime}}\left(p^{\prime}\right)\right)<\delta$ whenever $\operatorname{dist}\left(p, p^{\prime}\right)<\beta,\left|t-t^{\prime}\right|<\beta$, and $t, t^{\prime} \in[0,2 T]$. Let $\chi=(N, \tau, \eta, \gamma) \in \mathcal{C} h_{H}^{\beta, 2 T}(x, y)$ be a $(\beta, 2 T)$-chain from $x$ to $y$. Without loss of generality, we may assume that $\tau_{N+1}-\tau_{N} \leq 2 T$ by adding a trivial continuous-time jump at $\max \left(\tau_{N}+2 T, \tau_{N+1}-2 T\right)$ if necessary.

We would like to modify $\chi$ to get a nice $(\epsilon, T)$-chain. For each $k$ such that $0<\eta_{k}<N$ and $\tau_{\eta_{k}}-\tau_{\left(\eta_{k}-1\right)}<$ $T$, we will remove the continuous-time jump at $\tau_{\eta_{k}}$, continue on the execution prior to that jump, and return to a later point on the image of $\chi$ via a new jump. Let $u=\gamma_{\left(\eta_{k}-1\right)}\left(\tau_{\eta_{k}}\right)$ and $v=\gamma_{\eta_{k}}\left(\tau_{\eta_{k}}\right)$. Let $t=\tau_{\left(\eta_{k}+1\right)}-\tau_{\eta_{k}}$. One of two cases occurs: either (i) $t<2 T$ and $\tau_{\left(\eta_{k}+1\right)}$ is a reset jump, or (ii) $t \geq 2 T$. In the former case, we have two subcases based on whether $\widetilde{\varphi}^{t}(u)$ is either (a) defined or (b) undefined.

Case (i)(a): By the uniform continuity of the restriction of $\widetilde{\varphi} \operatorname{discussed}$ above, we have $\operatorname{dist}\left(\widetilde{\varphi}^{t}(u), \widetilde{\varphi}^{t}(v)\right)<$ $\delta$ since $\operatorname{dist}(u, v)<\beta$. Since $\tau_{\left(\eta_{k}+1\right)}$ is a reset jump, it follows that $\widetilde{\varphi}^{t}(v) \in \mathcal{G}$. Since $\widetilde{\varphi}^{t}(u)$ is defined, it follows that $\widetilde{\varphi}^{t}(u) \in U$ since $\delta<\operatorname{dist}(\mathcal{G}, \mathcal{X} \backslash U)$. Thus, $\operatorname{dist}\left(r \circ \rho\left(\widetilde{\varphi}^{t}(u)\right), r\left(\widetilde{\varphi}^{t}(v)\right)\right)<\epsilon / 2$. By the definition of $\chi$, we have $\operatorname{dist}\left(r\left(\widetilde{\varphi}^{t}(v)\right), \gamma_{\left(\eta_{k}+1\right)}\left(\tau_{\eta_{k}+1}\right)\right)<\beta<\epsilon / 2$. Thus, by the triangle inequality we have

$$
\operatorname{dist}\left(r \circ \rho\left(\widetilde{\varphi}^{t}(u)\right), \gamma_{\left(\eta_{k}+1\right)}\left(\tau_{\eta_{k}+1}\right)\right)<\epsilon,
$$

so we can replace the jump at $\tau_{\eta_{k}}$ by a modified jump at $\tau_{\left(\eta_{k}+1\right)}$ by extending the domain of $\gamma_{\left(\eta_{k}-1\right)}$ to $\left[\tau_{\left(\eta_{k}-1\right)}, \tau_{\left(\eta_{k}+1\right)}\right]$, i.e., by replacing $\gamma_{\left(\eta_{k}-1\right)}$ with $\left(\left[\tau_{\left(\eta_{k}-1\right)}, \tau_{\left(\eta_{k}+1\right)}\right] \ni s \mapsto \widetilde{\varphi}^{s-\tau_{\left(\eta_{k}-1\right)}}\left(\gamma_{\left(\eta_{k}-1\right)}\left(\tau_{\left(\eta_{k}-1\right)}\right)\right)\right)$. We obtain a modified chain after deleting $\tau_{\eta_{k}}$ from the sequence $\left(\tau_{j}\right)_{j=0}^{N}$, deleting $\eta_{k}$ from the sequence $\left(\eta_{j}\right)_{j=0}^{M}$, and reindexing the sequences accordingly.

Case (i)(b): Since there exists a Zeno or infinite execution starting at $u$ and since $\mathcal{G}$ is closed, there exists a unique "first impact time" $t_{0}<t<2 T$ such that $\widetilde{\varphi}^{t_{0}}(u) \in \mathcal{G}$. By our uniform continuity considerations, we have $\operatorname{dist}\left(\widetilde{\varphi}^{t_{0}}(u), \widetilde{\varphi}^{t_{0}}(v)\right)<\delta$. Since $\delta<\operatorname{dist}(\mathcal{G}, \mathcal{X} \backslash U)$, we have $\widetilde{\varphi}^{t_{0}}(v) \in U$. Thus,

$$
\operatorname{dist}\left(r\left(\widetilde{\varphi}^{t_{0}}(u)\right), r\left(\gamma_{\eta_{k}}\left(\tau_{\left(\eta_{k}+1\right)}\right)\right)\right)=\operatorname{dist}\left(r\left(\widetilde{\varphi}^{t_{0}}(u)\right), r \circ \rho\left(\widetilde{\varphi}^{t_{0}}(v)\right)\right)<\epsilon / 2 .
$$

Moreover, $\operatorname{dist}\left(r\left(\gamma_{\eta_{k}}\left(\tau_{\left(\eta_{k}+1\right)}\right)\right), \gamma_{\left(\eta_{k}+1\right)}\left(\tau_{\eta_{k}+1}\right)\right)<\beta<\epsilon / 2$. Thus, by the triangle inequality we have

$$
\operatorname{dist}\left(r\left(\widetilde{\varphi}^{t_{0}}(u)\right), \gamma_{\left(\eta_{k}+1\right)}\left(\tau_{\eta_{k}+1}\right)\right)<\epsilon
$$

so we can replace the jump at $\tau_{\eta_{k}}$ by a jump at $t_{0}+\tau_{\eta_{k}}$ by extending the domain of $\gamma_{\left(\eta_{k}-1\right)}$ to $\left[\tau_{\left(\eta_{k}-1\right)}, t_{0}+\tau_{\eta_{k}}\right]$, i.e., by replacing $\gamma_{\left(\eta_{k}-1\right)}$ with $\left(\left[\tau_{\left(\eta_{k}-1\right)}, t_{0}+\tau_{\eta_{k}}\right] \ni s \mapsto \widetilde{\varphi}^{s-\tau_{\left(\eta_{k}-1\right)}}\left(\gamma_{\left(\eta_{k}-1\right)}\left(\tau_{\left(\eta_{k}-1\right)}\right)\right)\right)$. We obtain a modified chain after replacing $\tau_{\left(\eta_{k}+1\right)}$ with $\left(t_{0}+\tau_{\eta_{k}}\right)$, deleting $\tau_{\eta_{k}}$ from the sequence $\left(\tau_{j}\right)_{j=0}^{N}$, deleting $\eta_{k}$ from the sequence $\left(\eta_{j}\right)_{j=0}^{M}$, and reindexing the sequences accordingly.

Case (ii): We want to replace the jump at $\tau_{\eta_{k}}$ with a jump at $\tau_{\eta_{k}}+T$. Since $\operatorname{dist}(u, v)<\beta$, our uniform continuity considerations imply that $\operatorname{dist}\left(\widetilde{\varphi}^{s}(u), \widetilde{\varphi}^{s}(v)\right)<\delta$ for all $s \in[0,2 T]$ such that the expression on the left is defined. Since $\widetilde{\varphi}^{s}(v)$ is defined for all $s \in[0,2 T]$ and since $\left.\mu\right|_{U}<T$ by our choice of $U$, it follows that $\widetilde{\varphi}^{s}(v) \in \mathcal{X} \backslash U$ for all $s \in[0, T]$. Since $\delta<\operatorname{dist}(\mathcal{G}, \mathcal{X} \backslash U)$, it follows that $\tilde{\varphi}^{s}(u)$ is also defined for all $s \in[0, T]$. Hence $\operatorname{dist}\left(\widetilde{\varphi}^{T}(u), \widetilde{\varphi}^{T}(v)\right)<\delta<\epsilon$, so we can replace the jump at $\tau_{\eta_{k}}$ with a jump at $T+\tau_{\eta_{k}}$ from $\widetilde{\varphi}^{T}(u)$ to $\gamma_{\eta_{k}}\left(T+\tau_{\eta_{k}}\right)$. We obtain a modified chain after extending the domain of $\gamma_{\left(\eta_{k}-1\right)}$ to $\left[\tau_{\left(\eta_{k}-1\right)}, T+\tau_{\eta_{k}}\right]$, replacing $\tau_{\eta_{k}}$ with $T+\tau_{\eta_{k}}$, and replacing $\gamma_{\eta_{k}}$ with its restriction $\left.\gamma_{\eta_{k}}\right|_{\left[T+\tau_{\eta_{k}}, \tau_{\left(\eta_{k}+1\right)}\right]}$.

After applying the procedure described above in Cases (i)(a-b) and (ii), we obtain an $(\epsilon, T)$-chain for which $\tau_{\eta_{k}}-\tau_{\left(\eta_{k}-1\right)} \geq T$ for all $k$ such that $0<\eta_{k}<N$. The resulting chain will be nice unless $\eta_{M}=N$ and $\tau_{N}-\tau_{N-1}<T$; if this is the case, we end up with an $(\epsilon, T)$-chain $\chi=(N, \tau, \eta, \gamma) \in \mathcal{C} h_{H}^{\epsilon, T}$ satisfying (I) 
$\tau_{\eta_{k}}-\tau_{\left(\eta_{k}-1\right)} \geq T$ for all $0<\eta_{k}<N$, (II) $\eta_{M}=N$, (III) $\tau_{N}-\tau_{N-1}<T$, and (IV) $\tau_{N+1}-\tau_{N} \leq 2 T$ (from the second paragraph of the proof). We call such chains almost-nice; note that $N \geq 2$ for an almost-nice chain. By the above argument, for any $\epsilon, T>0$ we can construct an $(\epsilon, T)$-chain from $x$ to $y$ which is either nice or almost-nice.

We now claim that, from the above, it follows that a nice $(\epsilon, T)$-chain between $x$ and $y$ exists. Suppose (to obtain a contradiction) that this is not the case. Then for each $n \in \mathbb{N}$, there exists an almost-nice $(1 / n, T)$-chain $\chi^{(n)}=\left(N^{(n)}, \tau^{(n)}, \eta^{(n)}, \gamma^{(n)}\right)$ from $x$ to $y$. For each $n$, define $t_{n}=\tau_{N^{(n)}}^{(n)}-\tau_{N^{(n)}-1}^{(n)}<T$ and $t_{n}^{\prime}=\tau_{N^{(n)}+1}^{(n)}-\tau_{N^{(n)}}^{(n)} \leq 2 T$. Similarly, let $u_{n}=r\left(\gamma_{N^{(n)}-2}^{(n)}\left(\tau_{N^{(n)}-1}^{(n)}\right)\right), v_{n}=\gamma_{N^{(n)}-1}^{(n)}\left(\tau_{N^{(n)}-1}^{(n)}\right)$, and $w_{n}=$ $\gamma_{N^{(n)}}^{(n)}\left(\tau_{N^{(n)}}^{(n)}\right)$. Since $\mathcal{X}$ is compact, after passing to a subsequence we may assume that $t_{n}, t_{n}^{\prime} \rightarrow t, t^{\prime} \in[0,2 T]$ and $u_{n}, v_{n}, w_{n} \rightarrow u, v, w \in \mathcal{X}$. Furthermore, each $\left(t_{n}, v_{n}\right)$ and $\left(t_{n}^{\prime}, w_{n}\right)$ belong to $\operatorname{dom}(\widetilde{\varphi})=\operatorname{cl}(\operatorname{dom}(\varphi))$, which is closed in $[0, \infty) \times \mathcal{X}$; hence $(t, v),\left(t^{\prime}, w\right) \in \operatorname{dom}(\widetilde{\varphi})$. Since also $\widetilde{\varphi}^{t_{n}}\left(v_{n}\right) \rightarrow w$ and $\widetilde{\varphi}^{\prime}\left(w_{n}\right) \rightarrow y$, we have $\widetilde{\varphi}^{t}(v)=w$ and $\widetilde{\varphi}^{t^{\prime}}(w)=y$ by continuity of $\widetilde{\varphi}$. Hence Lemma 1 implies that $\left(t+t^{\prime}, v\right) \in \operatorname{dom}(\widetilde{\varphi})$ and $\widetilde{\varphi}^{t+t^{\prime}}(v)=y$. Pick $n$ large enough so that $1 / n<\epsilon / 2$ and $\operatorname{dist}\left(v_{n}, v\right)<\epsilon / 2$. Since $\operatorname{dist}\left(u_{n}, v_{n}\right)<1 / n<\epsilon / 2$, the triangle inequality implies that $\operatorname{dist}\left(u_{n}, v\right)<\epsilon$. Thus we can modify the chain $\chi^{(n)}$ so that the jump occurring at $\tau_{N^{(n)}-1}^{(n)}$ is from $u_{n}$ to $v$, so that $\gamma_{N^{(n)}-1}^{(n)}$ is replaced with the arc

$$
\left[\tau_{N^{(n)}-1}^{(n)}, t+t^{\prime}+\tau_{N^{(n)}-1}^{(n)}\right] \ni s \mapsto \widetilde{\varphi}^{s-\tau_{N}^{(n)}-1}(v)
$$

terminating at $y$ and $\tau_{N^{(n)}}^{(n)}$ is replaced with $\left(t+t^{\prime}+\tau_{N^{(n)}-1}^{(n)}\right)$, and so that the final arc $\gamma_{N^{(n)}}^{(n)}$ and time $\tau_{N^{(n)}+1}^{(n)}$ are deleted from the sequences $\gamma^{(n)}, \tau^{(n)}$. Since $N \geq 2$ for an almost-nice chain, the resulting chain is a nice $(\epsilon, T)$-chain from $x$ to $y$ (consisting of $N-1$ arcs), which contradicts our assumption that a nice $(\epsilon, T)$-chain from $x$ to $y$ does not exist. This shows that $\widehat{\mathcal{C} h_{H}} \supseteq \mathcal{C} h_{H}$ and completes the proof.

Lemma 5. Let $H=(\mathcal{X}, \mathcal{F}, \mathcal{G}, \varphi, r)$ be an MHS satisfying Assumptions 1, 2, 3, and 4. Let $H^{\prime}=$ $\left(\mathcal{X}^{\prime}, \mathcal{F}^{\prime}, \mathcal{G}^{\prime}, \varphi^{\prime}, r^{\prime}\right)$ be the associated relaxed hybrid system equipped with any compatible extended metric making $H^{\prime}$ an MHS. Let $\iota: \mathcal{X} \rightarrow \mathcal{X}^{\prime}$ be the obvious embedding. Then for all $x, y \in \mathcal{X}$, we have

$$
\mathcal{C} h_{H}(x, y) \Longleftrightarrow \mathcal{C} h_{H^{\prime}}(\iota(x), \iota(y))
$$

Proof. Throughout the proof, let $d_{\mathcal{X}}$ be the extended metric for $\mathcal{X}$ and $d_{\mathcal{X}^{\prime}}$ be the extended metric for $\mathcal{X}^{\prime}$.

Let $\epsilon, T>0$ and $(x, y) \in \mathcal{C} h_{H}$. Since $\mathcal{X}$ is compact (Assumption 4$), \iota: \mathcal{X} \rightarrow \mathcal{X}^{\prime}$ is uniformly continuous, and hence there exists $\delta>0$ such that $u, v \in \mathcal{X}, d_{\mathcal{X}}(u, v)<\delta$ implies that $d_{\mathcal{X}^{\prime}}(\iota(u), \iota(v))<\epsilon$. Let $\chi=(N, \tau, \eta, \gamma) \in \mathcal{C} h_{H}^{\delta, T}(x, y)$. For each arc $\gamma_{j}$ of $\chi$, we define $\widetilde{\gamma}_{j}$ to be $\iota \circ \gamma_{j}$ if $\gamma_{j}$ ends in a continuous-time jump or is the final arc of $\chi$. Otherwise $\gamma_{j}$ ends in a reset jump, in which case we define $\widetilde{\gamma}_{j}$ to be the concatenation of $\iota \circ \gamma_{j}$ with the path $[0,1] \rightarrow \mathcal{X}^{\prime}$ defined by $t \mapsto \pi_{0}(t, z)$, where $z \in \mathcal{G}$ is the endpoint of $\gamma_{j}$ and $\pi_{0}$ is the quotient map of Def. 13. Then the collection of arcs $\widetilde{\gamma}_{j}$ defines a chain $\chi^{\prime} \in \mathcal{C} h_{H^{\prime}}^{\epsilon, T}(\iota(x), \iota(y))$.

For the converse, let $\epsilon, T>0$ and $(\iota(x), \iota(y)) \in \mathcal{C} h_{H^{\prime}}$. Let $\rho: U \subseteq \mathcal{X} \rightarrow \mathcal{G}$ be a flow-induced retraction. Shrinking $U$ if necessary, we may assume that $U$ is compact and that $\left.\mu\right|_{U}$ is strictly bounded above by $T \in(0, \infty)$, where the maximum flow time $\mu: \mathcal{X} \rightarrow[0,+\infty]$ is defined in Equation (1). Define $U^{\prime}:=$ $\iota(U) \cup \pi_{0}(\mathcal{G} \times[0,1]) \subseteq \mathcal{X}^{\prime}$, define the flow-induced retraction $\rho^{\prime}: U^{\prime} \rightarrow \mathcal{G}^{\prime}$ by $\rho^{\prime}(\iota(x)):=\pi_{0}(\rho(x), 1)$ and $\rho^{\prime}\left(\pi_{0}(z, t)\right):=\pi_{0}(z, 1)$, and define $\mu^{\prime}: U^{\prime} \rightarrow[0,1+T)$ by $\mu^{\prime}(\iota(u)):=1+\mu(u)$ and $\mu^{\prime}\left(\pi_{0}(z, t)\right):=1-t$. Let $\widetilde{\varphi}$ be the continuous extension of $\varphi^{\prime}$ to the closure $\operatorname{cl}\left(\operatorname{dom}\left(\varphi^{\prime}\right)\right)$ of $\operatorname{dom}\left(\varphi^{\prime}\right)$ in $[0, \infty) \times \mathcal{X}^{\prime}$ ensured by Assumptions 1, 2, and 3 and Lemma 1. Since $\mathcal{X}, \mathcal{G}$, and $U^{\prime}$ are compact and $\iota: \mathcal{X} \rightarrow \iota(\mathcal{X}) \subseteq \mathcal{X}^{\prime}$ is a homeomorphism, there exists $\delta>\zeta>0$ such that

$$
d_{\mathcal{X}^{\prime}}\left(\pi_{0}(\mathcal{G} \times[0,1]), \mathcal{X} \backslash \operatorname{int}\left(U^{\prime}\right)\right)>\zeta
$$

and

$$
\begin{aligned}
u, v \in \mathcal{X}, d_{\mathcal{X}^{\prime}}(\iota(u), \iota(v))<\delta & \Longrightarrow d_{\mathcal{X}}(u, v)<\epsilon \\
p, q \in U^{\prime}, d_{\mathcal{X}^{\prime}}(p, q)<\zeta & \Longrightarrow d_{\mathcal{X}^{\prime}}\left(r^{\prime} \circ \rho^{\prime}(p), r^{\prime} \circ \rho^{\prime}(q)\right)<\delta .
\end{aligned}
$$


By assumption there exists a chain $\chi^{(0)}=\left(N, \tau^{(0)}, \eta^{(0)}, \gamma^{(0)}\right) \in \widehat{\mathcal{C h}}_{H^{\prime}}^{\zeta, T+1}(\iota(x), \iota(y))$; recall from Def. 4 that $N \geq 1$. We will now modify the chain $\chi^{(0)}$ inductively. Fix $i \in\{0, \ldots, N-1\}$ and assume that, if $i \geq 1,{ }^{27}$ we have modified the first $(i+1) \operatorname{arcs}\left(\gamma_{0}^{(0)}, \ldots, \gamma_{i}^{(0)}\right)$ to obtain a chain $\chi^{(i)}=\left(N, \tau^{(i)}, \eta^{(i)}, \gamma^{(i)}\right) \in$ $\widehat{\mathcal{C h}}_{H^{\prime}}^{\delta, T+1}(\iota(x), \iota(y))$ such that (a) the sub-chain obtained by throwing away the first $(i+1)$ arcs of $\chi^{(i)}$ is either a single arc (if $i=N-1$ ) or a $(\zeta, T+1)$-chain, and (b) for all $j \in\{1, \ldots, i\}: \gamma_{j-1}^{(i)}\left(\tau_{j}^{(i)}\right) \notin \pi_{0}(\mathcal{G} \times[0,1))$ and $\gamma_{j}^{(i)}\left(\tau_{j}^{(i)}\right) \notin \pi_{0}(\mathcal{G} \times(0,1])$. If both $\gamma_{i}^{(i)}\left(\tau_{i+1}^{(i)}\right), \gamma_{i+1}^{(i)}\left(\tau_{i+1}^{(i)}\right) \in U^{\prime}{ }^{28}$ then we replace $\gamma_{i}^{(i)}$ with the arc

$$
\left[\tau_{i}^{(i)}, \tau_{i+1}^{(i)}+\mu^{\prime}\left(\gamma_{i}^{(i)}\left(\tau_{i+1}^{(i)}\right)\right)\right] \ni t \mapsto \widetilde{\varphi}^{t-\tau_{i}^{(i)}}\left(\gamma_{i}^{(i)}\left(\tau_{i}^{(i)}\right)\right)
$$

and $\gamma_{i+1}^{(i)}$ with the degenerate arc $\left(\left\{\tau_{i+1}^{(i)}+\mu^{\prime}\left(\gamma_{i}^{(i)}\left(\tau_{i+1}^{(i)}\right)\right)\right\} \ni t \mapsto r^{\prime} \circ \rho^{\prime}\left(\gamma_{i+1}^{(i)}\left(\tau_{i+1}^{(i)}\right)\right)\right)$. The upper bound $\mu^{\prime}(\cdot)<T$ and Equations (30), (32) can be used to show that, after redefining the sequences $\eta^{(i)}$ and $\tau^{(i)}$ accordingly, the result is a chain $\chi^{(i+1)}=\left(N, \tau^{(i+1)}, \eta^{(i+1)}, \gamma^{(i+1)}\right) \in \widehat{\mathcal{C h}}_{H^{\prime}}^{\delta, T+1}(\iota(x), \iota(y))$ such that (a) the sub-chain obtained by throwing away the first $(i+2) \operatorname{arcs}$ of $\chi^{(i+1)}$ is either empty (if $\left.i=N-1\right)$, a single arc (if $i=N-2)$, or a $(\zeta, T+1)$-chain and (b) for all $j \in\{1, \ldots, i+1\}: \gamma_{j-1}^{(i+1)}\left(\tau_{j}^{(i+1)}\right) \notin \pi_{0}(\mathcal{G} \times[0,1))$ and $\gamma_{j}^{(i+1)}\left(\tau_{j}^{(i+1)}\right) \notin \pi_{0}(\mathcal{G} \times(0,1])$.

Hence by induction we obtain a chain $\chi \in \widehat{\mathcal{C h}}_{H^{\prime}}^{\delta, T+1}(\iota(x), \iota(y))$ satisfying $\gamma_{i}\left(\tau_{i+1}\right) \notin \pi_{0}(\mathcal{G} \times[0,1))$ and $\gamma_{i}\left(\tau_{i}\right) \notin \pi_{0}(\mathcal{G} \times(0,1])$ for every arc of $\chi$. This implies that, if any arc of $\chi$ meets $\pi_{0}(\mathcal{G} \times[0,1])$, it must pass through $\pi_{0}(\mathcal{G} \times\{0\})$ and terminate at $\pi_{0}(\mathcal{G} \times\{1\})$. Deleting the portions of the arcs that pass through $\pi_{0}(\mathcal{G} \times[0,1])$, composing the resulting arcs with $\iota^{-1}: \iota(\mathcal{X}) \rightarrow \mathcal{X}$, and using (31), we finally obtain an $(\epsilon, T)$-chain from $x$ to $y$ for $H$. This completes the proof.

Lemma 6. Let $X$ be a compact metric space and $\Phi:[0, \infty) \times X \rightarrow X$ be a continuous semiflow. Consider $x, y \in X$ and fix $T>0$. If for every $\epsilon>0$ there exist $(\epsilon, T)$-chains from (i) $x$ to $y$ and (ii) $y$ to $x$, then $x$ and $y$ are chain equivalent.

Proof. It suffices to show that, for every $\epsilon>0$, there are $(\epsilon, 2 T)$-chains from (i) $x$ to $y$ and (ii) $y$ to $x$. By the compactness of $X$, the map $\Phi$ is uniformly continuous on $X \times[0,4 T]$. Hence there exists $\delta \in(0, \epsilon / 2)$ such that for all $a, b \in X$ and $t \in[0,4 T]$ we have $\operatorname{dist}\left(\Phi^{t}(a), \Phi^{t}(b)\right)<\epsilon / 2$ whenever $\operatorname{dist}(a, b)<\delta$.

Let $\chi=(N, \tau, \eta, \gamma) \in \mathcal{C} h^{\delta, T}(x, y)$. Without loss of generality, we may assume that $\tau_{i+1}-\tau_{i} \in[T, 2 T]$ for all $0 \leq i \leq N$. By concatenating $\chi$ with a $(\delta, T)$-chain from $y$ to $x$ followed by a $(\delta, T)$-chain from $x$ to $y$, we may assume that $N \geq 3$ (so that $\chi$ contains at least four arcs). Thus, there exist integers $N^{\prime} \geq 1$ and $r \in\{1,2\}$ with $N=2 N^{\prime}+r$.

Let $\tau^{\prime}=\left(\tau_{i}^{\prime}\right)_{i=0}^{N^{\prime}+1}$ be the sequence given by $\tau_{i}^{\prime}:=\tau_{2 i}$ for $0 \leq i \leq N^{\prime}$ and $\tau_{N^{\prime}+1}^{\prime}:=\tau_{N+1}$. We (uniquely) define the corresponding arcs $\gamma_{0}^{\prime}, \ldots, \gamma_{N^{\prime}}^{\prime}$ by their starting points $\gamma_{i}^{\prime}\left(\tau_{i}^{\prime}\right):=\gamma_{i}\left(\tau_{i}^{\prime}\right)$ for $0 \leq i \leq N^{\prime}$. Then by the triangle inequality the chain $\left(N^{\prime}, \tau^{\prime}, \eta^{\prime}, \gamma^{\prime}\right)$ (where $\eta^{\prime}=\left(0,1, \ldots, N^{\prime}\right)$ ) defines an $(\epsilon, 2 T)$-chain from $x$ to $y$.

Repeating the above argument with the roles of $x$ and $y$ reversed also yields an $(\epsilon, 2 T)$-chain from $y$ to $x$. This completes the proof.

\footnotetext{
${ }^{27}$ If $i=0$ we assume nothing, so that the base case of the induction argument is included in this one.

${ }^{28}$ Note that, if only one of $\gamma_{i}^{(i)}\left(\tau_{i+1}^{(i)}\right), \gamma_{i+1}^{(i)}\left(\tau_{i+1}^{(i)}\right)$ is in $U^{\prime}$, then (30) implies that it cannot belong to $\pi_{0}(\mathcal{G} \times[0,1])$.
} 